\title{
The role of B-glucan, plant stanols, and oxy(phyto)sterols in managing cardiovascular risk
}

Citation for published version (APA):

Theuwissen, E. (2008). The role of B-glucan, plant stanols, and oxy(phyto)sterols in managing cardiovascular risk. [Doctoral Thesis, Maastricht University]. Datawyse / Universitaire Pers Maastricht. https://doi.org/10.26481/dis.20081217et

Document status and date:

Published: 01/01/2008

DOI:

10.26481/dis.20081217et

Document Version:

Publisher's PDF, also known as Version of record

\section{Please check the document version of this publication:}

- A submitted manuscript is the version of the article upon submission and before peer-review. There can be important differences between the submitted version and the official published version of record.

People interested in the research are advised to contact the author for the final version of the publication, or visit the DOI to the publisher's website.

- The final author version and the galley proof are versions of the publication after peer review.

- The final published version features the final layout of the paper including the volume, issue and page numbers.

Link to publication

\footnotetext{
General rights rights.

- You may freely distribute the URL identifying the publication in the public portal. please follow below link for the End User Agreement:

www.umlib.nl/taverne-license

Take down policy

If you believe that this document breaches copyright please contact us at:

repository@maastrichtuniversity.nl

providing details and we will investigate your claim.
}

Copyright and moral rights for the publications made accessible in the public portal are retained by the authors and/or other copyright owners and it is a condition of accessing publications that users recognise and abide by the legal requirements associated with these

- Users may download and print one copy of any publication from the public portal for the purpose of private study or research.

- You may not further distribute the material or use it for any profit-making activity or commercial gain

If the publication is distributed under the terms of Article $25 \mathrm{fa}$ of the Dutch Copyright Act, indicated by the "Taverne" license above, 


\section{THE ROLE OF $\beta$-GLUCAN, PLANT STANOLS, AND OXY(PHYTO)STEROLS IN MANAGING CARDIOVASCULAR RISK}

ELKE THEUWISSEN 


\section{itutim}

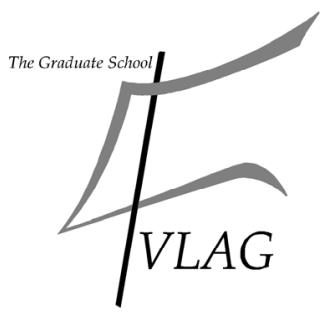

The studies in this thesis were performed within the Nutrition and Toxicology Research Institute Maastricht (NUTRIM), which participates in the Graduate School VLAG (Food Technology, Agrobiotechnology, Nutrition and Health Sciences), accredited by the Royal Netherlands Academy of Arts and Sciences.

Cover design: Philip Bosmans

Printed by Datawyse bv / Universitaire Pers Maastricht

(C) Elke Theuwissen, Maastricht 2008

ISBN 978-90-5278-784-8 


\section{The role of $\beta$-glucan, plant stanols, and oxy(phyto)sterols in managing cardiovascular risk}

\section{PROEFSCHRIFT}

ter verkrijging van de graad van doctor

aan de Universiteit Maastricht,

op gezag van de Rector Magnificus,

Prof. mr. G.P.M.F. Mols,

volgens het besluit van het College van Decanen,

in het openbaar te verdedigen

op woensdag 17 december 2008 om 14.00 uur

door

\section{Elke Theuwissen}

geboren te Leuven op 14 april 1979

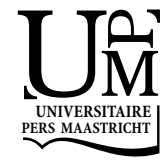




\section{Promotor:}

Prof. dr. ir. R.P. Mensink

\section{Copromotor:}

Dr. J. Plat

\section{Beoordelingscommissie:}

Prof. dr. ir. W.H.M. Saris (voorzitter)

Dr. F.J.P.H. Brouns

Prof. dr. em. G. Hornstra

Dr. D. Lütjohann (University of Bonn)

Prof. dr. C.D.A. Stehouwer

Financial support by the Netherlands Heart Foundation and Raisio for the publication of this thesis is gratefully acknowledged. 


\section{CONTENTS}

$\begin{array}{lll}\text { Chapter } 1 & \text { General introduction } & 7\end{array}$

Chapter $2 \quad$ Viscous soluble fibers and cardiovascular disease 19

Chapter 3 Simultaneous intake of oat $\beta$-glucan and plant stanols affects lipid metabolism in slightly hypercholesterolemic subjects 35

Chapter $4 \quad$ Consumption of oat $\beta$-glucan with or without plant stanols did not influence inflammatory markers in slightly hypercholesterolemic subjects

Chapter 5 Plant stanol consumption decreases serum triacylglycerols in subjects with overt hypertriglyceridemia

Chapter $6 \quad$ Oxyphytosterols increase the proportion of severe atherosclerotic lesions in LDLR+/- mice

Chapter $7 \quad$ General discussion

References

Summary

Samenvatting

Appendix

Dankwoord

Curriculum Vitae

Publications

Abbreviations 



\section{CHAPTER 1}

\section{General introduction}




\section{Introduction}

Despite remarkable improvements in its prevention and therapy, cardiovascular disease (CVD) remains the leading cause of morbidity and mortality in most Western countries. CVD is a term used to describe diseases of the heart and blood vessels, such as angina, myocardial infarction, diseases of the heart valves or muscle, heart failure, and stroke. Increased age, male sex, family history of CVD, smoking, unhealthy dietary habits, physical inactivity, obesity and overweight, hypertension, type 2 diabetes (DM2), and abnormal blood lipid levels [high serum levels of low-density lipoprotein (LDL) cholesterol, low levels of high-density lipoprotein (HDL) cholesterol, and/or high levels of triacylglycerols (TAG)] are some of the major risk factors for CVD.

\section{The pathogenesis of CVD}

Atherosclerosis is a slow, complex, chronic disease and the primary cause of CVD (1). The initiating step involves damage to the vascular endothelium, which can be triggered by smoking, hypertension, DM2, low-grade systemic inflammation, and hypercholesterolemia. The injured endothelium becomes more permeable, which results in accumulation of atherogenic LDL particles in the intimae. At this location, the lipoprotein particles can undergo oxidation. Oxidized LDL cholesterol stimulates endothelial expression of adhesion molecules, which attract monocytes to the arterial wall. In addition, oxidation of LDL cholesterol results in the expression of pro-inflammatory cytokines. These cytokines stimulate the endothelial adhesion of monocytes and their migration into the arterial wall. The monocytes, once recruited into the arterial intimae, transform into macrophages. These macrophages can take up oxidized LDL cholesterol through their scavenger receptors and become lipid-filled foam cells. Further damage occurs when surrounding smooth muscle cells (SMC) and endothelial cells start to secrete cytokines and growth factors. These molecules stimulate the proliferation and endothelial transmigration of SMC. At that time, SMC start synthesizing an extracellular matrix, in particular collagen. Cycles of accumulation of monocytes, migration, and proliferation of SMC and formation of fibrous tissue lead to further enlargement and restructuring in the atherosclerotic intimae. This eventually leads to the formation of a fibrous cap that covers the lipid-filled foam cells and consists of collagen-rich fibrous tissue, SMC, macrophages and T-lymphocytes. This is called the mature atherosclerotic plaque. The plaque protrudes into the arterial lumen, grows slowly over years, and can finally obstruct the artery. Moreover, the plaque can rupture, thereby exposing collagen and lipids to the blood stream. This leads to adherence and aggregation of platelets and initiates formation of a thrombus, which can block the artery and result in myocardial infarction and stroke. 


\section{TAG metabolism}

Abnormal blood lipid levels (high serum levels of LDL cholesterol, low levels of HDL cholesterol, and/or high levels of TAG) are an important risk factor for CVD. Over 95\% of dietary lipids are TAG; the remaining are phospholipids, free fatty acids, and cholesterol (present as esterified cholesterol). Dietary TAG are digested in the stomach and duodenum into monoacylglycerols and free fatty acids by gastric and pancreatic lipases. Monoacylglycerols, free fatty acids, and free cholesterol (unesterified) are then solubilized into micelles, which transport these lipids to the enterocytes. Once absorbed into the enterocytes, they are reassembled into TAG and packaged with cholesterol into chylomicrons (2). Chylomicrons [containing apolipoprotein (apo)B-48, apoC-II, and apoE] transport dietary TAG and cholesterol from within the enterocytes through the lymphatics into the circulation. In the circulation, TAG transported by chylomicrons undergo lipolysis through the action of lipoprotein lipase (LPL) at the surface of the capillary endothelium, liberating free fatty acids and glycerol. Glycerol and free fatty acids can be absorbed in the peripheral tissues, especially adipose tissue and muscle, for energy and storage. The TAG-depleted chylomicron remnants are then internalized by the hepatocytes expressing apoE receptors. TAG are also synthesized by the liver where they are packaged as very-low-density lipoproteins (VLDL; containing apoB-100, apoC-II, and apoE), and released into the circulation (2). Again like chylomicrons, VLDL particles circulate and encounter LPL expressed on endothelial cells, causing hydrolysis of the VLDL particle. A degraded or TAG-reduced VLDL particle is referred to as intermediate-density lipoprotein (IDL), reflecting the fact that decreasing TAG content increases particle density while decreasing particle size. Further, LPL works on IDL releasing more free fatty acids changing the particles to LDL. LDL particles are the primary carriers of cholesterol for delivery to all tissues.

The liver and intestine secrete HDL particles, which contain mainly phospholipids and apoA-1, with very little TAG or cholesterol (2). HDL particles scavenge unesterified (free) cholesterol in the circulation. Lecithin-cholesterol acyltransferase (LCAT), an enzyme associated with HDL, esterifies free cholesterol in the particle. Cholesteryl ester-transfer protein (CETP) transfers the LCAT-generated cholesteryl esters from HDL to apoB-100 containing lipoproteins (VLDL, IDL, and LDL) in exchange for TAG.

\section{Cholesterol metabolism}

Cholesterol is derived from both exogenous dietary sources and endogenous cholesterol synthesis. Under normal conditions, cholesterol homeostasis is maintained by balancing dietary cholesterol absorption and endogenous synthesis with excretion of bile acids and 
intestinal cholesterol (3). In the intestinal lumen, cholesterol is incorporated into mixed micelles and transported to the intestinal cells. From the intestinal cells, cholesterol can either be transported back into the lumen or into the circulation by means of chylomicrons. Similar to TAG, cholesterol is transported by the different lipoproteins (chylomicrons, $\mathrm{VLDL}$, IDL, LDL, and $\mathrm{HDL}$ ) in the circulation. The complete cholesterol absorption pathway has, however, not yet been fully defined. The Niemann-Pick C1 Like-1 (NPC1L1) transporter is suggested to play a role in the intestinal uptake of cholesterol and phytosterols (4). In addition, four members of the ATP-binding cassette (ABC) transporter family play an important role in modulating circulating and tissue cholesterol levels (5). ABCA1 and ABCG1 export excess cellular cholesterol into the HDL pathway and reduce cholesterol accumulation in macrophages. ABCG5 and ABCG8 are known to transport phytosterols back into the intestinal lumen, and some authors suggest that these transporters are involved in cholesterol efflux as well (6).

The liver is the major site of both synthesis and catabolism of cholesterol. Cholesterol is synthesized from acetyl-coenzyme A (acetyl-CoA) (3). The rate-limiting reaction for cholesterol synthesis is catalyzed by the microsomal enzyme 3-hydroxy-3-methyl-glutaryl (HMG)-CoA reductase. The activity of this enzyme depends on cellular cholesterol concentrations. Increasing dietary cholesterol intake, providing the cells with more exogenous cholesterol, suppresses hepatic HMG-CoA reductase synthesis and lowers LDL receptor expression. By controlling cholesterol synthesis and uptake, the cell can control its intracellular cholesterol levels.

The synthesis (and excretion) of bile acids is the major pathway by which the liver eliminates cholesterol (3). The adult human liver converts approximately $500 \mathrm{mg}$ cholesterol to bile acids on a daily basis. The primary bile acids, cholic acid and chenodeoxycholic acid, are synthesized from cholesterol in the liver by the hepatocytes. The initial and rate-limiting step in this process is the hydroxylation of cholesterol at the $\alpha$-position, which is catalyzed by cholesterol $7 \alpha$-hydroxylase. Before secretion into bile, the primary bile acids are conjugated to either glycine or taurine. In the terminal ileum, 90 to $95 \%$ of these bile acids are reabsorbed and returned to the liver. This process is known as the enterohepatic circulation (7). The remainder is lost to the colon, where the primary bile acids are transformed by bacterial metabolism into the secondary bile acids, deoxycholic acid and lithocholic acid. Some of the secondary bile acids are also reabsorbed and the rest is excreted with the faeces.

The levels of bile acids in the hepatocytes influence the activity of cholesterol $7 \alpha$ hydroxylase (8). Decreased cellular levels of bile acids, as may be the case with increased viscous soluble fiber intake, promote bile acid synthesis from cholesterol through decreased feedback inhibition on cholesterol $7 \alpha$-hydroxylase. Consequently, hepatic cholesterol levels decrease and HMG-CoA reductase activity and LDL-receptor activity increase. As a result, serum LDL cholesterol concentrations decrease (net effect). 


\section{Functional foods and CVD risk}

Dietary therapy has been shown to be effective in reducing CVD risk and mortality associated with high serum LDL cholesterol levels. Functional foods made to lower the atherogenic LDL particles, and therefore the risk for CVD, are gaining a prominent position in dietary guidelines (9). Such foods contain one or more functional components and therefore provide positive health effects beyond their traditional nutritional value. Examples of such food components with Food and Drug Administration (FDA)-approved health claims are the viscous soluble fiber $\beta$-glucan from oats (10) and barley (11) (see Chapter 2 and Figure 1) and plant sterols and stanols $(\mathbf{1 2}, 13)$ (see \$5).

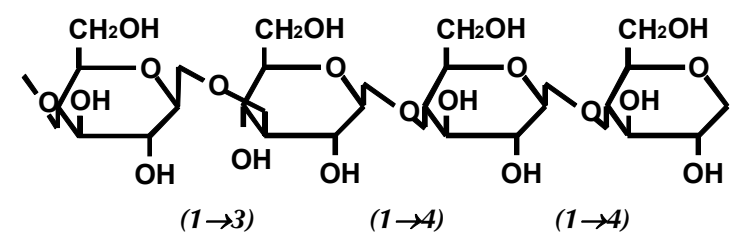

Figure 1: Structure of oat $\beta$-glucan.

Oat $\beta$-glucan is a polysaccharide composed of glucose molecules which are joined by $\beta-(1 \rightarrow 4)-$ and $\beta-(1 \rightarrow 3)-$ glycosidic bonds.

The combination of oat $\beta$-glucan and plant stanols in one food product may result in even larger reductions in serum LDL cholesterol concentrations, also because the underlying mechanisms of these two ingredients are suggested to be different. The study as described in Chapter $\mathbf{3}$ was therefore designed to investigate this.

\section{Plant sterols and stanols as functional foods}

Plant sterols are found in a wide variety of plant lipids, such as oils from vegetables, nuts, grains, seeds, wood pulp, and leaves. Plant stanols, which are saturated plant sterols, occur in even smaller quantities in many of the same sources. Plant sterols and stanols, collectively referred to as phytosterols, are essential components of plant cell membranes and structurally resemble cholesterol. The human body cannot synthesize these components and therefore all phytosterols present, originate from the diet. In Western countries, people consume on average 160 to $360 \mathrm{mg} / \mathrm{d}$ of phytosterols as part of their normal healthy diet 
(14). Typical diets commonly provide sitosterol, campesterol, and stigmasterol, along with smaller amounts of plant stanols, like sitostanol (Figure 2).
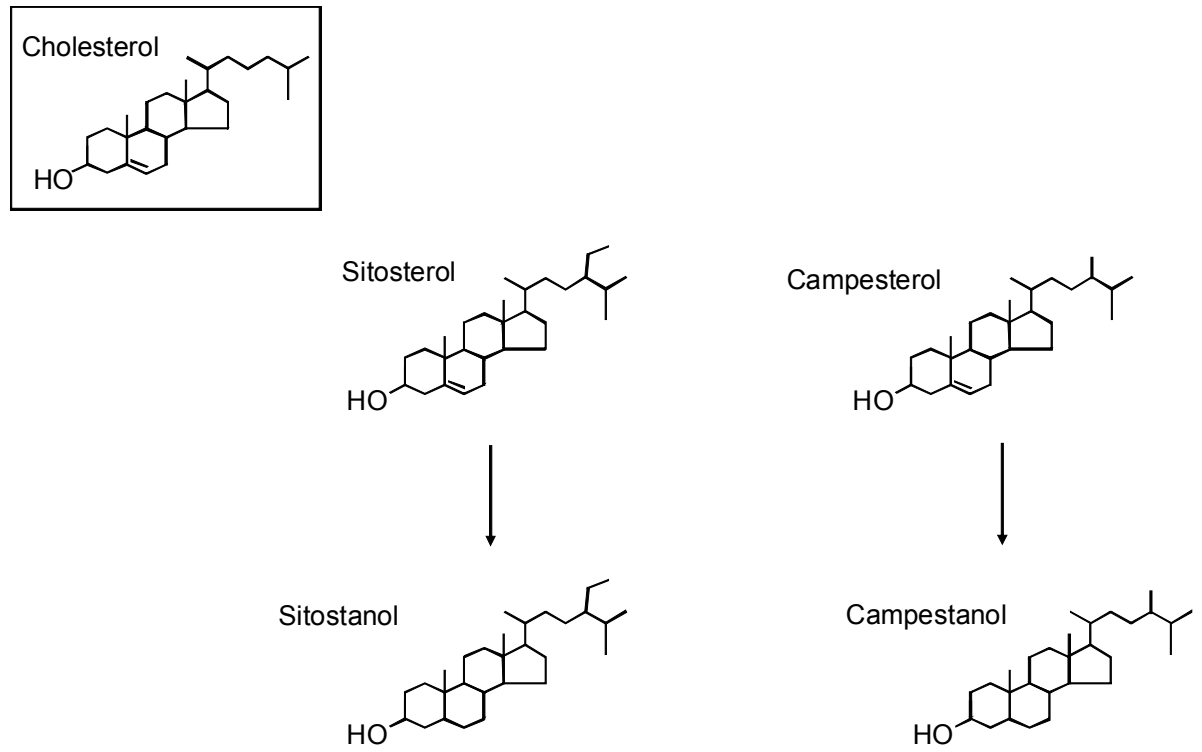

Figure 2: Structures of phytosterols.

Plant sterols and stanols are essential components of plant cell membranes and structurally resemble cholesterol.

Under normal circumstances, the Western diet contains almost equal amounts of cholesterol and phytosterols. However, the net absorption of plant sterols and stanols is much lower than that of cholesterol $[<5 \%$ versus 55 to $60 \%$ (15)]. Despite their initial absorption from the intestinal tract using the same pathway as cholesterol, phytosterols are almost completely returned to the intestinal lumen through specific transporters (16). Consequently, serum levels of phytosterols are very low and in the range from 2.5 to $30 \mu \mathrm{mol} / \mathrm{L}$ (17).

The cholesterol-lowering potential of phytosterols has been known for over 50 years. Although people consume plant sterols and stanols every day, the amounts are often not large enough to have significant cholesterol-lowering effects (14). By modifying phytosterols structurally to form sterol and stanol esters, larger amounts can be easily incorporated into high-fat foods (spreads, dressings) without loosing their effectiveness in lowering serum LDL cholesterol concentrations. Phytosterol esters are also added to a wide variety of low-fat products, such as low-fat yoghurt, low-fat milk, bread, and cereals. A recent 
meta-analysis concluded that daily consumption of 2.0 to $2.4 \mathrm{~g}$ phytosterols in ester form lowers on average serum LDL cholesterol concentrations by $8.9 \%$ (14). The FDA has authorized the claim that foods containing at least $0.65 \mathrm{~g}$ per serving of phytosterol esters (daily total intake $\geq 1.3 \mathrm{~g}$ ), as part of a diet low in saturated fat and cholesterol, can reduce the risk of CVD (12).

This hypocholesterolemic effect of plant sterols and stanols can be (partly) explained by their ability to compete with cholesterol for incorporation into micelles, particles that transport lipids and cholesterol into the intestinal mucosa (18). This competition reduces dietary and biliary cholesterol absorption in the gastrointestinal tract. However, recent findings suggest that phytosterols also can enter the intestinal cells and promote cholesterol efflux back into the intestinal lumen through a yet unknown mechanism (18). The decrease in cholesterol absorption results in a compensatory increase in endogenous cholesterol synthesis (19). Additionally, receptor-mediated cholesterol uptake becomes upregulated (19). The overall effect is a reduction in serum LDL cholesterol concentrations (Figure 3).
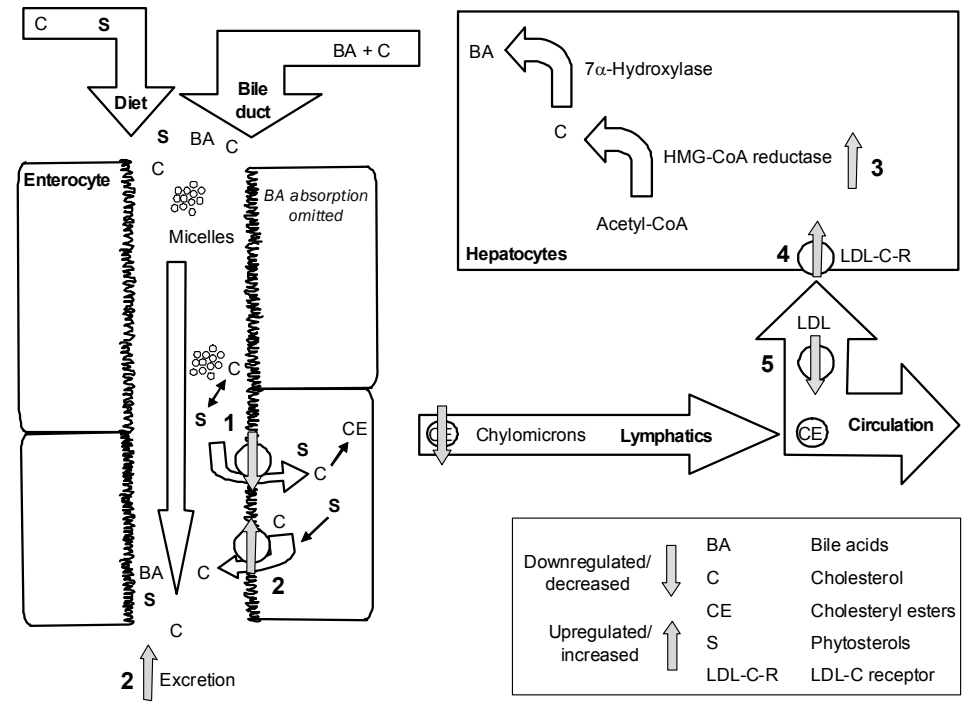

Figure 3: Postulated hypocholesterolemic mechanism of phytosterols.

Phytosterols may compete with cholesterol for incorporation into micelles, which reduces cholesterol absorption into the enterocytes (1). Phytosterols may also enter the enterocytes and promote cholesterol efflux back into the intestinal lumen (2). This results in a compensatory increase in endogenous cholesterol synthesis (3). Additionally, receptormediated cholesterol uptake becomes upregulated (4). The overall effect is a reduction in serum LDL cholesterol levels (5). 
Phytosterols and their esters are Generally Recognized as Safe (GRAS) food substances, as a history of intake has not demonstrated any harmful health effects (20). Nevertheless, a first potential concern is that they may also reduce absorption of fat-soluble vitamins (18). However, after several years of use in Europe and the U.S, no impaired vitamin status or related adverse effects have been reported. Recently, however, it has been hypothesized that increased serum plant sterol levels probably may increase CVD risk. This hypothesis is based on the observation that patients with a rare recessive genetic lipid storage disease, known as phytosterolemia, have an uncontrolled sterol absorption, severely elevated serum plant sterol concentrations, low endogenous cholesterol synthesis, xanthomas, and premature atherosclerosis (15). However, results from epidemiological studies in the general population are conflicting and there is no evidence at present that marginally increased serum plant sterol levels can increase CVD risk (15). One possibility by which plant sterols may be potentially atherogenic is through oxidative modification (see next paragraph).

\section{Oxy(phyto)sterols and CVD risk}

Oxidized forms of cholesterol (also known as oxysterols; Figure 4) are suggested to be atherogenic and accordingly may also play an active role in the pathogenesis of CVD (21). Oxysterols arise in tissues as a result of enzymatic (during cholesterol catabolism) or nonenzymatic (in vivo oxidation; e.g. 27-hydroxycholesterol) oxidation reactions and are also obtained from dietary sources (dairy products, egg, meat, fish, bakery products) (21). In vitro, oxysterols perturb several aspects of cellular cholesterol homeostasis (including cholesterol biosynthesis, esterification, and efflux), impair vascular reactivity, are cytotoxic, and induce apoptosis (21). However, there is no direct evidence yet in humans that oxysterols are causally linked to atherogenesis. Nevertheless, animal studies have shown that oxysterols in the diet can accelerate fatty streak lesion formation $(22,23)$. 


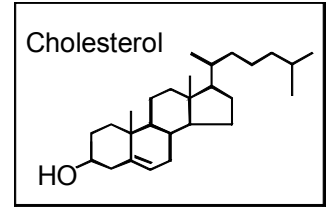

$3 \beta, 5 \alpha, 6 \beta$-trihydroxycholestane<smiles>CC(C)CCC(C)C12CCC3C4CC5(CC(O)C(O)C5(O)C4CC31)C2(C)C</smiles>

27-hydroxycholesterol

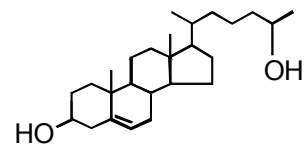

$7 \alpha$-hydroxycholesterol

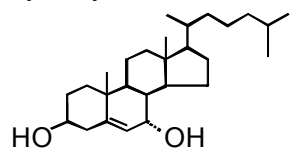

5,6-epoxycholesterol

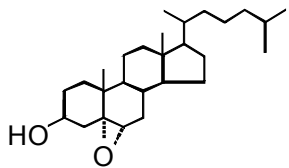

24S-hydroxycholesterol

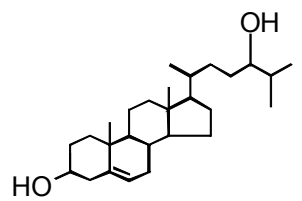

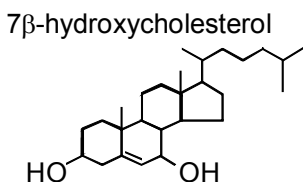

7-ketocholesterol<smiles>CC(C)CCC(C)C1CCC2C1CCC1C2C(=O)C2CC(O)CCC21C</smiles>

25-hydroxycholesterol

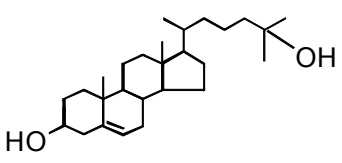

Figure 4: Structures of oxysterols.

Plant sterols structurally resemble cholesterol and the presence of one or more unsaturated bonds makes phytosterols susceptible to oxidation as well. Small amounts of oxidized phytosterols (oxyphytosterols) have been found in the diet (coffee, fried potatoes, wheat flour, and vegetable oils) (24), and in serum from phytosterolemic patients (25) and healthy subjects (26). Animal studies have shown that oxyphytosterols are absorbed from the diet and accumulate in serum, liver, and aorta (27). Further, in vitro studies have shown that oxyphytosterols exhibit toxic effects in cultured macrophages similar to those of oxysterols (28). However, it is unknown whether oxyphytosterols are atherogenic, as has been suggested for oxysterols. In Chapter 6, the results are discussed from a randomized, controlled animal study, in which we investigated the effects of oxysterols and oxyphytosterols side-by-side on lipid metabolism and atherosclerotic lesion development using heterozygous LDL receptor-deficient (LDLR+/-) mice.

\section{Inflammation and CVD risk}

Inflammation is the process by which the body responds to injury or infections. Inflammation in the vessel wall plays an essential part in both the development of atherosclerosis 
and the rupture of atherosclerotic plaques (1). All stages, like initiation, growth, and complication of the atherosclerotic plaque, can be considered to be an inflammatory response to injury. Recent findings suggest that statins, commonly prescribed LDL cholesterol-lowering drugs, reduce this inflammation, indicating an additional beneficial effect. Examination of the possibility to interfere with the inflammatory part in atherosclerosis by diet or functional foods in particular, currently receives a lot of attention.

Besides their hypocholesterolemic effects, in vitro and animal studies have suggested that oat $\beta$-glucan and phytosterols may also affect inflammatory parameters (29-37). Data from human studies concerning these effects are however limited and inconsistent. To explore whether the effects of oat $\beta$-glucan and plant stanols on CVD risk may be mediated in part by affecting the inflammatory process, we first examined in Chapter $\mathbf{4}$ their effects on serum high-sensitivity CRP (hs-CRP) concentrations in slightly hypercholesterolemic subjects. Hs-CRP is considered a marker for low-grade systemic inflammation and is positively associated with an increased risk for future cardiovascular events (38). Since leukocyte reactivity is easily triggered by modified LDL cholesterol, potential changes may be more evident in hypercholesterolemic subjects. We therefore determined the effects of oat $\beta$-glucan and plant stanols on leukocyte function in these subjects (Chapter 4). This was studied ex vivo by measurement of inflammatory cytokine (IL-6, IL-8, TNF- $\alpha$ ) production after lipopolysaccharide (LPS) stimulation by isolated peripheral blood mononuclear cells (PBMC) and whole blood. II-6, IL-8, and TNF- $\alpha$ are pro-inflammatory markers involved in the development of atherosclerotic lesions. Finally, we investigated the effects of oat $\beta$-glucan and plant stanols on the expression of 84 atherosclerosis-related genes in PBMC to enhance the understanding of underlying mechanisms of these dietary compounds (Chapter 4).

\section{Hypertriglyceridemia and CVD risk}

The link between high serum LDL cholesterol levels and CVD has been clearly established. However, evidence is accumulating that high levels of TAG, also known as hypertriglyceridemia, and low levels of HDL cholesterol are also related to CVD (39). TAG are major lipids in chylomicrons (transport of TAG of dietary origin) and VLDL particles (transport of TAG synthesized by the liver). These particles are closely related to the metabolism of other lipoproteins, including HDL. High TAG and low HDL concentrations often occur together, frequently with normal serum concentrations of LDL cholesterol. This lipid abnormality is a fundamental characteristic of patients with the metabolic syndrome, a condition strongly associated with the risk to develop DM2 and CVD.

Interestingly, a recent meta-analysis has indicated that consumption of plant stanols may decrease serum TAG concentrations, especially in subjects with high serum TAG concen- 
trations (40). This meta-analysis was based on the individual data from 5 trials carried out at our Department and included almost 400 subjects. The reason that these effects have not been observed in previous studies may be due to a lack of statistical power, as effects have been marginal in subjects with normal serum TAG concentrations. We therefore designed a study to evaluate the effects of plant stanols on the lipoprotein profile in a population especially selected for elevated serum TAG concentrations (Chapter 5).

\section{OUTLINE OF THE THESIS}

This thesis focuses on the role of different dietary components (oat $\beta$-glucan, plant stanols, and oxy(phyto)sterols) in managing CVD risk. Chapter 2 describes the relationship between viscous soluble fibers and CVD. Chapter 3 was designed to investigate the effects of a simultaneous intake of 2 functional foods, oat $\beta$-glucan and plant stanols, on lipid metabolism in mildly hypercholesterolemic volunteers. In Chapter 4, we investigated the effects of these functional ingredients on serum hs-CRP levels in the same study population. We also determined their effects on leukocyte function, by measuring the production of proinflammatory cytokines (IL-6, IL-8, and TNF- $\alpha$ ) by isolated PBMC and whole blood after LPS stimulation (Chapter 4). In addition, we studied the effects of oat $\beta$-glucan and plant stanols on the expression of 84 atherosclerosis-related genes in PBMC (Chapter 4). Chapter 5 describes the effects of plant stanols on serum TAG concentrations in subjects with hypertriglyceridemia. In Chapter 6, we investigated the effects of oxysterols and oxyphytosterols on lipid metabolism and atherosclerotic lesion development in LDLR+/- mice. 



\section{CHAPTER 2}

\section{Viscous soluble fibers and cardiovascular disease}

Elke Theuwissen and Ronald P. Mensink

Based on:

Physiol Behav. 2008; 94(2): 285-92 


\section{ABSTRACT}

Review One well-established way to reduce the risk of developing cardiovascular disease (CVD) is to lower serum LDL cholesterol levels by reducing saturated fat intake. However, the importance of other dietary approaches, such as increasing the intake of viscous soluble fibers is increasingly recognized. Well-controlled intervention studies have now shown that four major viscous soluble fiber types - $\beta$-glucan, psyllium, pectin and guar gum - effectively lower serum LDL cholesterol concentrations, without affecting HDL cholesterol or triacylglycerol concentrations. It is estimated that for each additional gram of viscous soluble fiber in the diet serum total and LDL cholesterol concentrations decrease by $-0.028 \mathrm{mmol} / \mathrm{L}$ and $-0.029 \mathrm{mmol} / \mathrm{L}$, respectively. Despite large differences in molecular structure, no major differences existed between the different types of viscous soluble fiber, suggesting a common underlying mechanism. In this respect, it is most likely that viscous soluble fibers lower the (re)absorption of in particular bile acids. As a result hepatic conversion of cholesterol into bile acids increases, which ultimately leads to increased LDL uptake by the liver. Additionally, epidemiological studies suggest that a diet high in viscous soluble fiber is inversely associated with the risk of CVD. These findings underlie current dietary recommendations to increase viscous soluble fiber intake. 


\section{INTRODUCTION}

Despite remarkable improvements in its prevention and therapy, cardiovascular disease (CVD) remains the leading cause of morbidity and mortality in the United States and most Western countries. One well-established way to reduce the risk of developing CVD is to lower serum LDL cholesterol levels by making dietary changes. In addition to reducing saturated fat and cholesterol intake, and increasing cis-unsaturated fat intake, the importance of other dietary approaches, such as increasing the intake of viscous soluble fibers has become increasingly recognized.

Dietary fiber is a collective term for a variety of plant substances that are resistant to digestion by human gastrointestinal enzymes. Dietary fiber can be classified as either soluble or insoluble in water (41). Cellulose, lignin, some pectins, and some hemicelluloses are insoluble fibers. Vegetables and cereal grains are especially rich in insoluble fiber, with the highest amounts in wheat and corn. Insoluble fiber is responsible for increased stool bulk and helps to regulate bowel movements. The natural gel-forming fibers, such as $\beta$ glucans, gums, mucilages (e.g. psyllium), pectins, and some hemicelluloses are soluble. Foods rich in soluble fiber are dried beans, oats, barley, and some fruits and vegetables. The mean total daily fiber intake amongst adults in most industrialized countries is well below $25 \mathrm{~g}$, the minimal amount recommended by various health organizations. Of total dietary fiber intake, approximately $20 \%$ is soluble and $80 \%$ is insoluble (42).

Further research has revealed that solubility does not reliably predict the physiological effects of fiber. It is the viscosity of dietary fiber that seems to be important for its physiological properties. Some fibers form very viscous solutions or gels in water (43). This property is linked to the ability of these fibers to slow the emptying of the stomach, delay the absorption of some nutrients in the small intestine, and lower serum cholesterol levels. Viscous fibers include $\beta$-glucans, pectins, some gums (e.g. guar gum) and mucilages (e.g. psyllium). Cellulose, lignin, and some hemicelluloses are nonviscous fibers. However, the terms "water-soluble" and "water-insoluble" are still used by many nutrition and health care professionals, as well as the FDA for nutrition labeling.

The main purpose of this review is to discuss the effects of different viscous soluble fiber types - $\beta$-glucan, psyllium, pectin, and guar gum - on cholesterol metabolism and cardiovascular risk. We limited the review to those viscous soluble fibers for which more than five intervention studies were published. First, we focused on the hypocholesterolemic effects of these four major viscous soluble fiber types. Next, possible mechanisms underlying the cholesterol-lowering effects were described. Finally, we summarized the epidemiological studies that contrasted the intake of total dietary fiber with that of cereal fiber, an important source of viscous soluble fiber. 


\title{
HYPOCHOLESTEROLEMIC EFFECT OF VISCOUS SOLUBLE FIBERS
}

\author{
$\beta$-Glucan
}

$\beta$-Glucan is a viscous soluble fiber found in cereals, in particular oats and barley, as well as in yeast, bacteria, algae, and mushrooms. In fact, one of the richest sources of $\beta$-glucan is the cell wall of baker's yeast Saccharomyces Cerevisae. Regardless of its source, $\beta$-glucan is a polysaccharide composed of glucose molecules. In bacteria and algae, however, the glucose molecules are joined by $\beta$ - $(1 \rightarrow 3)$-glycosidic bonds forming a linear chain, while in yeast and mushrooms the glucose molecules are joined by $\beta-(1 \rightarrow 3)$ - and $\beta-(1 \rightarrow 6)$ glycosidic bonds, and in oats and barley by $\beta-(1 \rightarrow 4)$ - and $\beta-(1 \rightarrow 3)$-glycosidic bonds. Thus, $\beta$-glucan from these latter sources is composed of branched chains. Functional foods enriched with $\beta$-glucan from mainly oats are widely available on the market to decrease serum LDL cholesterol concentrations.

In 1963, De Groot et al. were the first to report that in healthy men 3-week consumption of $300 \mathrm{~g}$ of bread, containing $140 \mathrm{~g}$ of rolled oats, decreased serum total cholesterol concentrations by $11 \%(44)$. Since that time, many studies have been carried out on the cholesterol-lowering effects of oats, which were later found to be due to its $\beta$-glucan content (45). Results of several uncontrolled metabolic ward trials have been summarized by Anderson et al. and reductions in serum total cholesterol from 13 to $26 \%$ were reported (46). These reductions were mainly found in the LDL cholesterol fraction. Although many of these metabolic ward studies showed impressive lipid reductions (47-49), trials with free-living subjects reported considerably more variability in lipid responses. Some studies found reductions greater than $10 \%(50-52)$, but a few studies demonstrated virtually no benefit (53-55). Inconsistencies in the reported effects of oat products may be due to several factors, such as mode of administration, or differences in solubility or molecular weight. Kerckhoffs et al., for example, investigated the effects of $\beta$-glucan in bread and cookies (study 1 ) and in orange juice (study 2 ). In study 1 , forty-eight mildly hypercholesterolemic subjects received bread and cookies rich in wheat fiber or rich in $\beta$-glucan $(5.9 \mathrm{~g} / \mathrm{d}$ ) for 3 weeks. Serum LDL cholesterol concentrations decreased slightly by $3 \%$ after $\beta$-glucan consumption. This change however did not reach statistical significance. In study 2 , the same sources and daily amounts (as in study 1) of control fiber and $\beta$-glucan were provided. Twenty-five subjects now consumed orange juice containing either wheat fiber or $\beta$-glucan for 2 weeks. It was found that serum LDL cholesterol concentrations decreased significantly by $6.7 \%$ (56). The authors concluded that the food matrix and/or food processing can have detrimental effects on the hypocholesterolemic properties of oat $\beta$ glucan. Solubility and molecular weight of $\beta$-glucan may also influence its hypocholes- 
terolemic effects. Indeed, it has been postulated that the viscosity of $\beta$-glucan in the intestinal tract, which is positively related to its solubility in water and molecular weight, is an important determinant of its LDL-cholesterol lowering effects. Highly soluble $\beta$-glucan, with moderate to high molecular weight, may reduce serum LDL cholesterol levels better than $\beta$-glucan with a low solubility and low molecular weight. This difference in effect has been explained by the assumption that a higher intestinal viscosity lowers the reabsorption of bile acids, leading to an increased excretion of bile acids. Increased bile acid excretion promotes bile acid synthesis from cholesterol, which increases LDL cholesterol uptake by the liver.

Although a few individual studies showed virtually no effects, several meta-analyses concluded that viscous soluble fiber from oat products effectively lowered serum total and LDL cholesterol concentrations (57-59). In the most recent meta-analysis, Brown et al. estimated that the intake of $1 \mathrm{~g}$ of viscous soluble fiber from oats lowered total and LDL cholesterol concentrations with $-0.037 \mathrm{mmol} / \mathrm{L}$ and $-0.032 \mathrm{mmol} / \mathrm{L}$, respectively (59) (Figure 1).

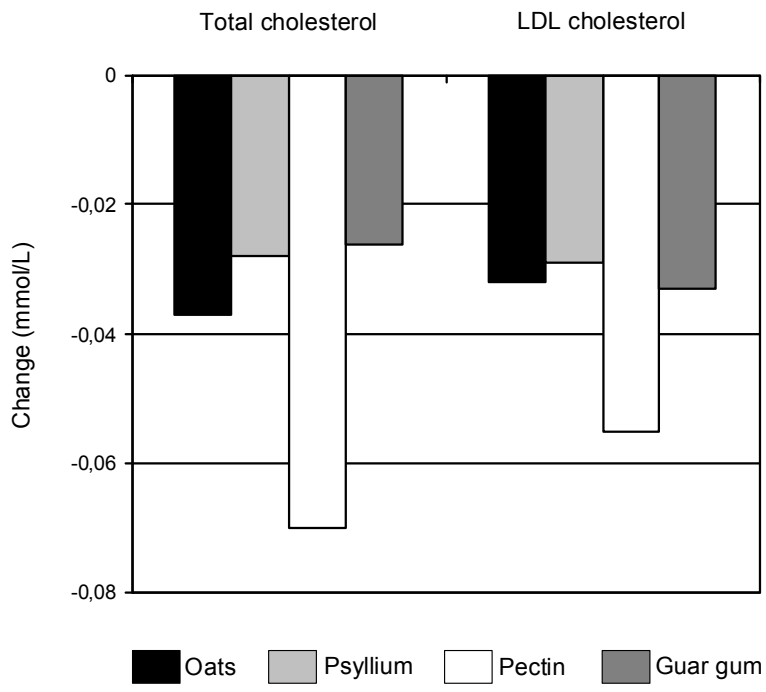

Figure 1: Effects of each gram of four major viscous soluble fiber types on changes in total and LDL cholesterol concentrations $(\mathrm{mmol} / \mathrm{L})$ [Adapted from (59)] 
Studies were carried out with healthy volunteers, hypercholesterolemic subjects, hypertensive subjects, diabetics, and subjects with an ileostoma (60-66). Oat was incorporated in breads, (hot) cereals, muffins, flakes, biscuits, shakes, and in drinks. After reviewing 37 studies, the Food and Drug Administration (FDA) approved in January 1997 a health claim that "Viscous soluble fiber from oatmeal, as part of a low saturated fat, low cholesterol diet, may reduce the risk of heart disease". The FDA determined that $3 \mathrm{~g} / \mathrm{d}$ of $\beta$-glucan must be consumed to achieve a clinically relevant serum cholesterol-lowering effect. The whole oat-containing food should provide at least $0.75 \mathrm{~g}$ of viscous soluble fiber per serving (10). Elevated postprandial lipidemia is also a marker for CVD risk. As fasting serum lipid concentrations are only slightly changed after consumption of oat bran, it has been suggested that postprandial lipid and lipoprotein responses after a meal may be more relevant to explain the effects of increased oat consumption on cardiovascular health (67). Yet, the results on the effects on postprandial lipoproteins are inconclusive. Many of the published postprandial studies investigated the effect of oat bran added to a test meal and found significant beneficial effects on lipid responses $(45,68,69)$. However, some trials did not observe any significant postprandial effects of oat bran consumption $(47,55)$, while a few studies even reported significantly higher postprandial TAG concentrations $(67,70)$.

Another important source of $\beta$-glucan is barley. While oats contain around $4 \% \beta$-glucan, 5 to $10 \% \beta$-glucan is present in barley. Despite its naturally high $\beta$-glucan content, fewer trials have investigated barley fiber since barley is not significantly consumed across regions. Nevertheless, the FDA recently concluded, based on the totality of available scientific evidence, that whole grain barley and dry milled barley products, such as flakes, grits, flour, and pearled barley, are appropriate sources of $\beta$-glucan viscous soluble fiber to claim that they reduce the risk of CVD (11). The effects of this viscous soluble fiber on serum cholesterol concentrations have been examined in healthy normocholesterolemic and hypercholesterolemic subjects (71-76). $\beta$-Glucan intake varied from 3 to $12 \mathrm{~g}$. The test foods were made from barley bran, barley flakes, barley flour, and barley concentrate. Reductions in LDL cholesterol varied from 0 to $17 \%$. Like for oats, not all studies reported improvements in the serum lipid profile (72). It was suggested that the lack of effect, at least in part, could be a consequence of structural changes in $\beta$-glucan due to processing of the barley into a highly enriched $\beta$-glucan product. Freezing, storage, or baking of the product may have influenced the efficacy of the fiber.

Finally, $\beta$-glucan is abundant in yeast as used in breweries and bakeries. After processing, the yeast contains up to $85 \%$ of $\beta$-glucan. In contrast to $\beta$-glucan from oats, yeast-derived $\beta$-glucan has a low viscosity and low solubility in water. Still, it may improve the serum lipoprotein profile, as demonstrated by Nicolosi et al. (77). After a 3-week period on their usual diets, 15 obese, hypercholesterolemic men consumed $15 \mathrm{~g}$ of fiber added to an orange juice for 8 weeks. The authors found that, compared with baseline, fiber consumption significantly reduced serum total cholesterol levels by $6 \%$. Serum LDL concentrations 
declined significantly by $8 \%$ and HDL concentrations increased significantly with $9 \%$ after 8 weeks. Serum TAG concentrations were unaffected. It should be noted, however, that this study lacked a proper control group. Obviously, more research is needed to confirm the cholesterol-lowering effects of this source of $\beta$-glucan and its potential beneficial effects on HDL cholesterol concentrations.

\section{Psyllium}

Psyllium is a gel-forming mucilage from the Plantago ovata plant, a native herb from parts of Asia, Mediterranean regions of Europe, and North Africa. Mucilage is a gelatinous substance, related to natural gums. The term psyllium is used interchangeably for the seed husk, the seed, and the entire plant. Psyllium is cultivated, because the seed husk is a rich source of viscous soluble fiber, known as psyllium hydrophilic mucilloid, psyllium hydrocolloid, and psyllium seed gum. The bioactive fraction in psyllium is a fiber composed of a highly branched arabinoxylan. The backbone consists of xylose units, while arabinose and xylose form the side chains. In contrast to the arabinoxylans in cereal grains that are extensively fermented, psyllium possesses an as yet unidentified structural feature that hinders its fermentation by typical colonic microflora (78). Nowadays, psyllium is incorporated into foods, such as ready-to-eat cereals, for its LDL cholesterol-lowering properties.

In 1965, Garvin et al. published the first study to suggest that psyllium lowered serum total cholesterol concentrations. In a non-placebo controlled trial, subjects received $9.6 \mathrm{~g} / \mathrm{d}$ of psyllium in addition to their habitual diet (psyllium group) or a high-egg diet (control group). After eight weeks, serum total cholesterol concentrations were decreased by $9 \%$ in the psyllium group. On the high-egg diet, however, a reduction of $7 \%$ was observed as well (79). Since then, psyllium has been investigated in numerous clinical trials. In these studies, it was found that psyllium lowered serum total and LDL cholesterol levels in hypercholesterolemic subjects, subjects with non-insulin dependent diabetes mellitus, and in subjects receiving lipid-lowering drug therapy $(59,80)$. Furthermore, effects of psyllium did not depend on the amount of fat or cholesterol in the diet (59). In these studies, mainly psyllium-enriched cereals and powder-preparations were used (59, 80, 81). The efficacy of psyllium was confirmed by the meta-analysis from Brown et al. (59), that concluded that each gram of viscous soluble fiber from psyllium lowered total and LDL cholesterol levels by $-0.028 \mathrm{mmol} / \mathrm{L}$ and $-0.029 \mathrm{mmol} / \mathrm{L}$, respectively (Figure 1). No effects on serum levels of HDL cholesterol or triacylglycerols (TAG) were observed (59). Based on the total body of evidence, the FDA has approved in 1998 a health claim for psyllium that "A food product containing viscous soluble fiber from psyllium seed husk, consumed as part of a diet low in saturated fat and cholesterol, may reduce the risk of heart disease". For this, $7 \mathrm{~g}$ of viscous 
soluble psyllium fiber must be consumed daily. Foods carrying the health claim must provide at least $1.7 \mathrm{~g}$ of viscous soluble fiber from psyllium per reference amount habitually consumed (82).

\section{Pectin}

Pectin is a gum found in many plants, but mainly in citrus fruits (oranges, lemons, grapefruits) and apples. During ripening, pectin changes from an insoluble substance to a much more soluble component. Pectin is a linear polysaccharide containing about 300 to 1,000 monosaccharide units, mainly D-galacturonic acid plus some polysaccharides. The Dgalacturonic acid molecules are linked together by $\alpha-(1 \rightarrow 4)$-glycosidic bonds. The galacturonic acid residues in pectin can be esterified with methyl groups. There are different types of pectin. Pectin in which more than $50 \%$ of the galacturonic acid residues are esterified is called "high-methoxyl" pectin. Pectin in which less than $50 \%$ of the galacturonic acid residues are esterified is called "low-methoxyl" pectin (83). Pectin is widely used in the food industry as a gelling agent to create a gelled texture to foods, but it also has hypocholesterolemic properties. Because of its strong gelling properties, it is difficult to incorporate high amounts of pectin into foods.

In 1961, Keys et al. already published the first human study that suggested that pectin supplementation lowered serum total cholesterol concentrations. Twenty-four healthy subjects consumed a series of 3-week experimental diets and 3-week standard diets for a total of 12 weeks. Consumption of biscuits with pectin $(15 \mathrm{~g} / \mathrm{d})$ reduced total cholesterol by $5 \%$ (84). Since then, many studies have confirmed the hypocholesterolemic effects of pectin. Pectin lowered serum cholesterol levels in hypercholesterolemic subjects, normocholesterolemic subjects, and in subjects with essential hypertension (59). Different sources of pectin, such as guava fruit, prunes, grapefruit gelatin capsules, and citrus pectin desserts were all effective. However, not all trials showed that pectin supplementation was associated with decreased serum cholesterol levels (85). Mahalko et al. investigated persons with type II diabetes in 2 studies. In the first study, daily consumption of $26 \mathrm{~g}$ of dehydrated powdered apple incorporated into seven slices of bread lowered serum total and LDL cholesterol concentrations by $4.7 \%$ and $8.3 \%$, respectively. In the second study, $52 \mathrm{~g}$ of the same fiber source $(26 \mathrm{~g}$ incorporated into seven slices of bread plus $26 \mathrm{~g}$ into other food products), significantly increased serum total and LDL cholesterol by $6.4 \%$ and $10.8 \%$, respectively, compared with the control period (white bread). However, only 6 subjects completed the second study (86). When all the available studies were however summarized, Brown et al. reported in their meta-analysis that viscous soluble fiber from pectin effectively lowered total and LDL cholesterol concentrations. The range of effects on total cholesterol varied from -16 to $-5 \%$. On average, each gram of viscous soluble fiber 
from pectin decreased total and LDL cholesterol concentrations by $-0.070 \mathrm{mmol} / \mathrm{L}$ and $0.055 \mathrm{mmol} / \mathrm{L}$, respectively (Figure 1). Pectin did not significantly alter serum levels of HDL cholesterol or TAG (59).

\section{Guar gum}

Guar, or Indian cluster bean Cyamopsis tetragonolobus, is a drought-tolerant annual leguminous plant originating from India and Pakistan. Guar gum is the primary marketable product of the plant. Guar gum is a galactomannan storage polysaccharide made up of polymers comprising about 10,000 molecules. The fiber consists of a $(1 \rightarrow 4)$-linked- $\beta$-Dmannopyranose backbone with $(1 \rightarrow 6)$-linked- $\alpha$-D-galactose side-chains. The overall ratio of mannose to galactose is around 2:1. Guar gum is used as a thickener and stabilizer in food and pharmaceutical products. It hydrates easily in cold water giving a highly viscous solution. Guar gum is also used to treat constipation and to decrease serum LDL cholesterol concentrations.

Forty years ago, Fahrenbach et al. found that guar administration effectively lowered serum total cholesterol levels in normocholesterolemic subjects (87). Since then, this serum lipidlowering effect of guar gum has been investigated in a large number of human trials. The results of these studies were consistent in that total and LDL cholesterol were effectively decreased by guar gum supplementation, whereas HDL cholesterol and TAG remained unchanged. From their meta-analysis, Brown et al. estimated that one gram of viscous soluble fiber from guar gum lowered serum total and LDL cholesterol by $-0.026 \mathrm{mmol} / \mathrm{L}$ and $-0.033 \mathrm{mmol} / \mathrm{L}$, respectively (59) (Figure 1). Guar gum lowered total cholesterol concentrations in healthy volunteers, hyperlipidemic subjects, and in subjects with diabetes mellitus (59). Also in subjects receiving oral hypoglycaemic drugs, antihypertensive medication, or lipid-lowering drugs, the cholesterol-lowering effects were still present (59). Numerous forms of guar gum were used, including powder, (coated) granules, and depolymerized forms. Guar gum was incorporated into various foodstuffs, such as bread, crackers, pasta and snack bars $(59,88)$. Human studies further showed that the physiologic effects of guar gum were not decreased after treatment with galactomannase, an enzyme that degrades the mannose and galactose units (89). An important benefit of using depolymerized guar gum is that much larger quantities of the galactomannan can be incorporated into food products without serious loss of sensory qualities (88). 


\section{Conclusion}

Since 1960, many studies have documented the hypocholesterolemic effects of four major viscous soluble fiber types - $\beta$-glucan, psyllium, pectin, and guar gum - in humans. High intakes of these viscous soluble fibers have been associated with significant decreases in serum total and LDL cholesterol concentrations. HDL cholesterol and TAG levels were not affected by viscous soluble fiber intake. Based on a recent meta-analysis, a mean reduction of $-0.029 \mathrm{mmol} / \mathrm{L}$ in serum LDL cholesterol concentrations can be expected for each additional gram intake of viscous soluble fiber. No major differences were reported between the different sources of viscous soluble fiber, suggesting a common mechanism underlying the hypocholesterolemic effects.

\section{HYPOCHOLESTEROLEMIC MECHANISM OF VISCOUS SOLUBLE FIBERS}

The exact mechanism by which viscous soluble fibers lower serum LDL cholesterol levels is not known. Evidence suggests that viscous soluble fibers may interfere with lipid and/or bile acid metabolism. Other suggested mechanisms include: the inhibition of hepatic cholesterol synthesis by fermentation products, and delayed absorption of carbohydrates leading to increased insulin sensitivity.

\section{Interference with lipid and/or bile acid metabolism}

Evidence suggests that some viscous soluble fibers may bind bile acids during formation of micelles in the intestinal lumen $(90,91)$. These observations led to the hypothesis that viscous soluble fibers may increase excretion of bile acids. Alternatively, it has been suggested that viscous soluble fibers may form a thick unstirred water layer in the intestinal lumen. This layer may act as a physical barrier, thereby decreasing the (re)absorption of fats, including cholesterol and bile acids. This would lead to an increased fecal output of these two components. As a result, hepatic conversion of cholesterol into bile acids increases, hepatic pools of free cholesterol decrease and - to reach a new steady-state endogenous cholesterol synthesis increases. This leads to increased activities of $7 \alpha$ hydroxylase and HMG-CoA reductase to compensate for the losses of bile acids and cholesterol from the liver stores. Furthermore, hepatic LDL cholesterol receptors become upregulated to restore hepatic cholesterol stores, which ultimately leads to decreased serum LDL cholesterol concentrations (Figure 2) (59). 

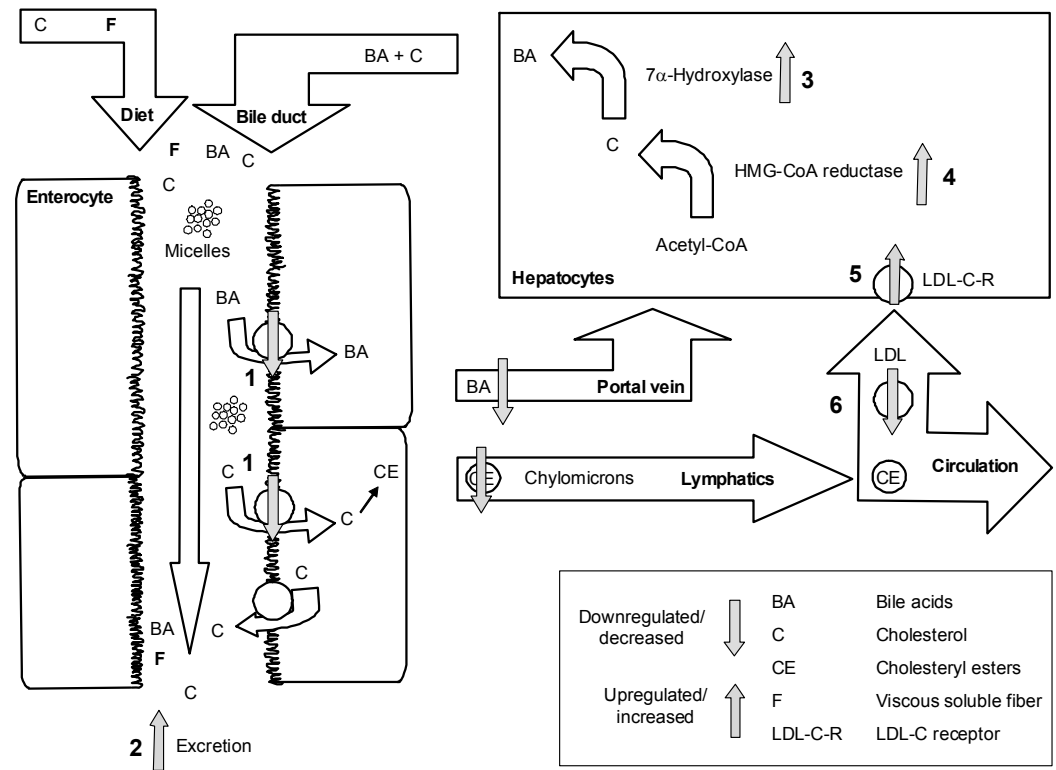

Figure 2: Postulated hypocholesterolemic mechanism of viscous soluble fibers.

The viscous water-soluble fibers form a thick unstirred water layer in the intestinal lumen, thereby decreasing the (re)absorption of cholesterol and bile acids (1). This leads to an increased fecal output of these two components (2). As a result hepatic conversion of cholesterol into bile acids increases (3), hepatic pools of free cholesterol decrease and endogenous cholesterol synthesis increases (4). In addition, hepatic LDL cholesterol receptors are upregulated to re-establish hepatic free cholesterol stores (5). These processes will ultimately lead to decreased serum LDL cholesterol levels (6).

Several human studies have explored the effects of viscous soluble fibers on bile acid excretion. In hypercholesterolemic men, who were given $100 \mathrm{~g}$ of oat bran for 10 days, a reduction in serum cholesterol levels and an increase in fecal bile acid excretion were observed (47). In another study, ileostomy subjects were served for 2 consecutive days 4 different breads in random order. The breads were mainly made from oat bran, oat bran treated with $\beta$-glucanase, barley, or wheat flour. The 24-hours excretion of bile acids was significantly higher in the oat bran-diet period than in the other 3 periods (92). The decreased molecular weight of the oat bran treated with $\beta$-glucanase and of the barley $\beta$ glucan may explain the lack of effect of these latter 2 diets. Andersson et al. investigated whether the increased fecal bile acid excretion after consumption of $\beta$-glucan from oat bran indeed increased bile acid synthesis. For this, healthy subjects received either $75 \mathrm{~g}$ wheat bran or $75 \mathrm{~g}$ oat bran for breakfast in a randomized crossover study. It was found that oat bran almost doubled the serum concentrations of $7 \alpha$-hydroxy-4-cholesten-3-one 
( $7 \alpha-\mathrm{OHC})$ shortly after intake (93). $7 \alpha-\mathrm{OHC}$ is made from $7 \alpha$-hydroxycholesterol, and serum $7 \alpha-\mathrm{OHC}$ concentrations strongly correlate with the activity of the key enzyme cholesterol $7 \alpha$-hydroxylase. In a study with hypercholesterolemic subjects, muesli enriched with $5 \mathrm{~g} \beta$-glucan also increased $7 \alpha-\mathrm{OHC}$ concentrations compared to the control muesli. Additionally, campesterol concentrations decreased and lathosterol concentrations increased, suggesting decreased cholesterol absorption and increased cholesterol synthesis, respectively (63). Also, Naumann et al. found decreased cholesterol absorption and increased cholesterol synthesis in hypercholesterolemic subjects, as measured by the same markers (64). In ileostomy subjects (94), $75 \mathrm{~g}$ oat bran with $11.6 \mathrm{~g}$ native $\beta$-glucan increased bile acid excretion within 24 hours of consumption compared to 4.5 g hydrolysed $\beta$-glucan, and this increase was paralleled by a rise in serum concentrations of $7 \alpha$ OHC. In addition, native $\beta$-glucan consumption decreased cholesterol absorption and increased lathosterol concentrations.

Effects from short-chain fatty acids produced by fermentation in the colon

Viscous soluble fibers escape absorption in the small bowel and are fermented in the large bowel by colonic bacteria. This fermentation results in the production of short-chain fatty acids (SCFA), such as acetate, propionate, and butyrate. Butyrate is primarily metabolized by colonic mucosal cells, while acetate and propionate are rapidly absorbed. It has been hypothesized that production of SCFA may influence cholesterol synthesis. However, this hypothesis mainly originates from animal studies, while properly controlled intervention studies in humans are scarce. Besides, data on the effects of SCFA production in the colon on serum lipids in humans are still conflicting.

Bridges et al. compared the effects of oat bran and wheat bran on serum SCFA concentrations in 20 hypercholesterolemic men. Oat bran decreased serum cholesterol concentrations by $12.8 \%$, whereas wheat bran had no effect. Serum acetate values were significantly higher in subjects fed the oat bran diet when compared with the wheat bran diet (95). These findings suggest that SCFA responses may contribute to the hypocholesterolemic effects of oat bran. In addition, serum acetate and propionate concentrations were negatively related to serum total and LDL concentrations in a cross-sectional study with healthy men and women. The relationships, however, disappeared in males and became less strong in females after adjusting for age and body mass index (96). On the contrary, rectal infusion studies showed that colonic acetate was incorporated into serum cholesterol and acutely raised the concentration of serum lipids, and that these effects were blocked by propionate $(97,98)$. Nevertheless, rectal infusion studies have been criticized as being nonphysiologic (96). 
Reduced insulin stimulation of hepatic lipogenesis

Viscous soluble fibers may lower the rate of glucose absorption, due to increased intestinal viscosity (99). The lowered postprandial glucose concentrations result in lowered postprandial insulin concentrations and decreased insulin-stimulated hepatic HMG-CoA activity and hence cholesterol synthesis. Changes in other hormones may also contribute to the decreased insulin concentrations after viscous soluble fiber consumption. Whether these hormonal changes result in lowered fasting serum LDL cholesterol concentrations has, however, never been demonstrated in humans.

\section{Conclusion}

Although the exact mechanism of viscous soluble fiber action is not known, the most likely mechanism is that viscous soluble fibers lower the (re)absorption of in particular bile acids. As a result hepatic conversion of cholesterol into bile acids increases, hepatic pools of free cholesterol decrease and - to reach a new steady-state - endogenous cholesterol synthesis increases. This leads to increased activities of $7 \alpha$-hydroxylase and HMG-CoA reductase to compensate for the losses of bile acids and cholesterol from the liver stores. Furthermore, hepatic LDL cholesterol receptors become upregulated to re-establish hepatic cholesterol stores, which ultimately leads to decreased serum LDL cholesterol concentrations (net effect).

\section{EPIDEMIOLOGY}

More than 30 years ago, Trowell formulated the fiber hypothesis related to coronary heart disease (CHD). He stated, "Data have been produced to support a hypothesis that high consumption of natural starchy carbohydrates, taken with their full complement of fiber, is protective against hyperlipidemia and ischemic heart disease (IHD)" (100). After these initial observations, a large number of epidemiological studies have strengthened this hypothesis. Results of prospective cohort studies published after 1995 are summarized in Table 1. Only those studies are presented that contrasted the intake of total dietary fiber with that of cereal fiber. From this table, it is evident that in most studies cereal fiber intake better predicts cardiovascular risk than total fiber intake. In the studies that also examined relationships between other sources of viscous soluble fiber (fruits, vegetables) with CVD risk, results were less consistent (101-105). This however may be because fruit and vegetable intake was too low to find any consistent findings. Taken together, however, these findings led to the approval by the FDA of the health claim: "Diets low in saturated 
fat and cholesterol and rich in fruits, vegetables, and grain products that contain some types of dietary fiber, particularly viscous soluble fiber, may reduce the risk of heart disease" $(10,11,82,106)$.

\section{CONCLUSION}

Many well-controlled intervention studies have shown that four major viscous soluble fiber types - $\beta$-glucan, psyllium, pectin and guar gum - effectively lower serum LDL cholesterol concentrations, without affecting HDL cholesterol or TAG concentrations. Furthermore, epidemiological studies suggest that a diet high in viscous soluble fiber protects against CVD. These findings underlie current dietary recommendations to further increase viscous soluble fiber intake. 


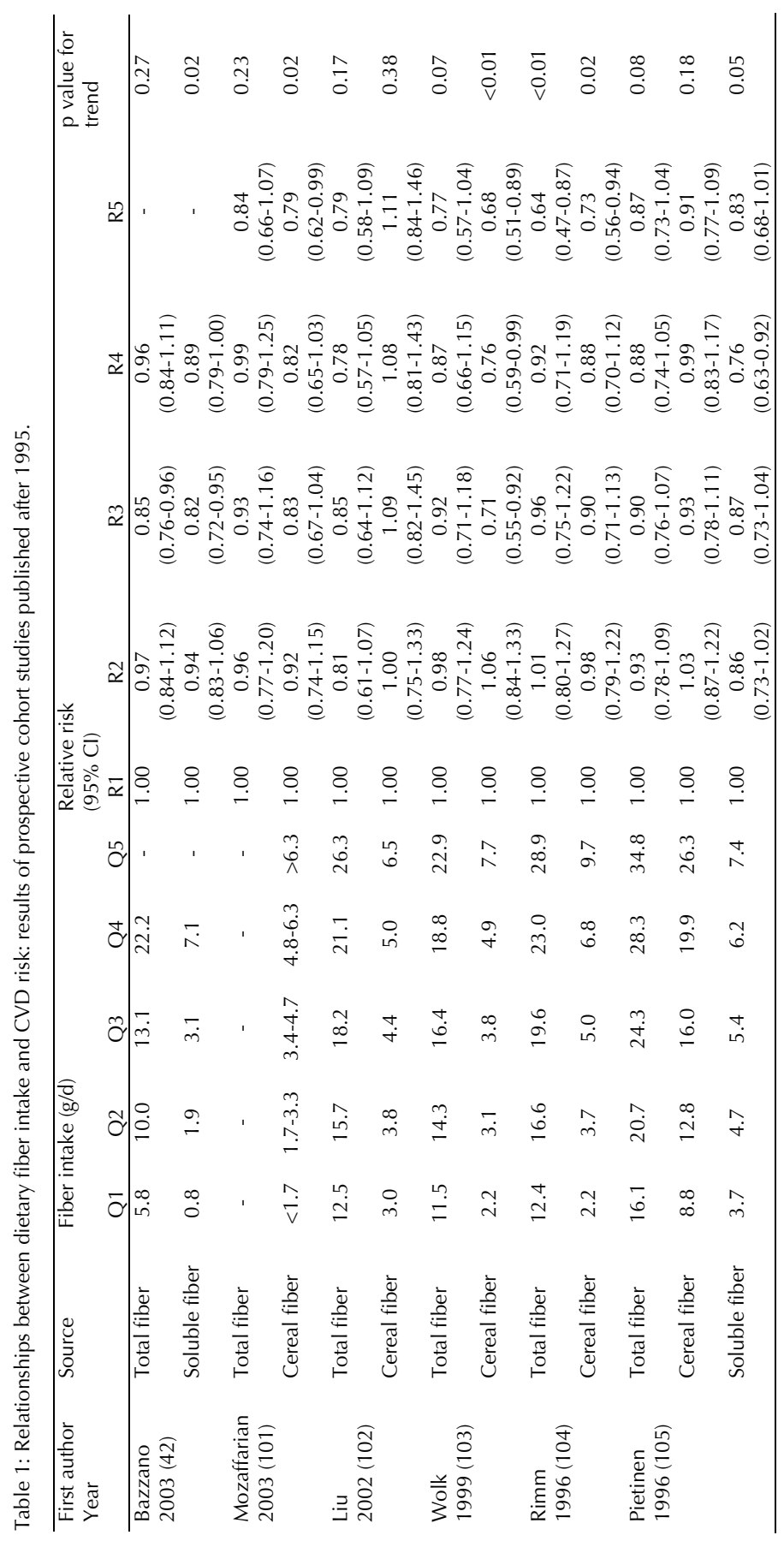





\section{CHAPTER 3}

\section{Simultaneous intake of}

\section{oat $\beta$-glucan and plant stanols affects lipid metabolism in slightly hypercholesterolemic subjects}

Elke Theuwissen, Ronald P. Mensink 


\section{ABSTRACT}

Objective Intake of food products rich in the viscous soluble fiber $\beta$-glucan from oats and products enriched with plant stanols lower serum LDL cholesterol concentrations. Combining two functional food ingredients into one food product may achieve additional reductions in serum LDL cholesterol levels.

Study design In a randomized, controlled, 3-period crossover study, 40 mildly hypercholesterolemic men and women received for 4 weeks in random order twice-daily muesli, which provided in total: $5 \mathrm{~g}$ control fiber from wheat (control muesli), $5 \mathrm{~g} \beta$-glucan from oats (oat $\beta$-glucan enriched muesli), or $5 \mathrm{~g}$ oat $\beta$-glucan plus $1.5 \mathrm{~g}$ plant stanols (combination muesli).

Results The oat $\beta$-glucan enriched muesli decreased serum LDL cholesterol concentrations by $5.0 \%$ compared to the control muesli $(p=0.013)$. The combination muesli significantly reduced LDL cholesterol levels by $9.6 \%$ compared to the control muesli $(p<0.001)$, and by $4.4 \%$ compared to the oat $\beta$-glucan enriched muesli $(p=0.036$ ). Serum HDL cholesterol and triacylglycerol concentrations did not differ between the 3 treatments. Compared to the control muesli, the oat $\beta$-glucan enriched muesli increased bile acid synthesis $(p=0.043$ ) and decreased cholesterol absorption $(\mathrm{p}=0.011)$. Addition of plant stanols did not influence bile acid synthesis, but decreased cholesterol absorption and raised cholesterol synthesis, compared to the control muesli $(p<0.001$ and $p=0.016$, respectively) and the oat $\beta$-glucan muesli ( $p=0.004$ and $p=0.814$, respectively). The combination muesli decreased serum concentrations of sitostanol compared to the control muesli $(p=0.010)$. Plasma concentrations of lipid-soluble antioxidants did not differ between the 3 treatments.

Conclusions Oat $\beta$-glucan enriched muesli effectively lowered serum LDL cholesterol concentrations. Addition of plant stanols to the oat $\beta$-glucan enriched muesli further lowered serum LDL cholesterol levels, although effects were slightly less than predicted. 


\section{INTRODUCTION}

LDL cholesterol-lowering functional foods are gaining a prominent position in dietary guidelines. Such foods contain one or more components, and therefore provide positive health effects beyond their traditional nutritional value (9). Examples of such food components with approved FDA-health claims are the viscous soluble fiber $\beta$-glucan from oats (10), and plant sterols and stanols (12).

$\beta$-Glucans are viscous soluble fibers, which are found in a wide variety of products such as oats, barley, and yeast (107). Regardless of their source, all $\beta$-glucans are polysaccharides composed of glucose molecules. Although the exact mechanism explaining the cholesterol-lowering effects of $\beta$-glucan is not known, the most likely explanation is that viscous soluble fibers lower the (re)absorption of bile acids $(47,68,92,108)$. As a result, hepatic conversion of cholesterol into bile acids increases, hepatic pools of free cholesterol decrease and - to reach a new steady-state - endogenous cholesterol synthesis increases (68). Furthermore, hepatic LDL cholesterol receptors become upregulated to re-establish hepatic cholesterol stores, which ultimately leads to decreased serum LDL cholesterol concentrations (109). The FDA has approved the claim that consumption of viscous soluble fiber ( $\geq 3 \mathrm{~g} / \mathrm{d}$ ) from whole oats, as part of a diet low in saturated fat and cholesterol, may reduce the risk of heart disease (10). In the most recent meta-analysis, it was estimated that $1 \mathrm{~g}$ of viscous soluble fiber from oats lowered serum LDL cholesterol concentrations by $0.037 \mathrm{mmol} / \mathrm{L}(59)$.

Plant sterols are found in a wide variety of plant lipids, such as oils from vegetables, nuts, grains, seeds, wood pulp, and leaves. Plant stanols, which are saturated plant sterols, occur in even smaller quantities in many of the same sources. Plant sterols and stanols, collectively referred to as phytosterols, are essential components of plant cell membranes and structurally resemble cholesterol. Typical diets commonly provide sitosterol, campesterol, and stigmasterol, along with smaller amounts of plant stanols like sitostanol. Phytosterols decrease the absorption of dietary and endogenously derived cholesterol in the intestine (110). Accordingly, endogenous cholesterol synthesis increases (111) and receptormediated cholesterol uptake becomes upregulated, which ultimately leads to decreased serum LDL cholesterol concentrations (19). The FDA has therefore authorized the claim that foods containing at least $0.65 \mathrm{~g}$ per serving of plant sterol and stanol esters (daily total intake $\geq 1.3 \mathrm{~g}$ ), as part of a diet low in saturated fat and cholesterol, may reduce the risk of heart disease (12). As of January 2003, the FDA recognized that the scientific literature supports expanding the health claim to include free forms of plant sterols and stanols, and to include a wider range of products, including low-fat products. The FDA further stated that the lowest effective daily intake of free sterols is $0.8 \mathrm{~g} / \mathrm{d}$ (13). A recent meta-analysis concluded that daily consumption of 2.0 to $2.4 \mathrm{~g}$ of plant sterols or stanols lowers on average serum LDL cholesterol concentrations by $8.9 \%$ (14). 
The combination of oat $\beta$-glucan and plant stanols in a food product may result in even larger reductions in serum LDL cholesterol concentrations, also because the underlying mechanisms of these two ingredients are suggested to be different. In addition, a combined intake may lead to a larger product variety or food product formulations that are more easily accepted by consumers. However, a concurrent intake of these two food components has not been examined before. We therefore decided to investigate the effects of the simultaneous intake of oat $\beta$-glucan and plant stanols on lipid metabolism in mildly hypercholesterolemic volunteers.

\section{MATERIALS AND METHODS}

\section{Study population}

Healthy men and women, aged 18 to $65 \mathrm{y}$, with slightly elevated serum total cholesterol concentrations, were recruited from Maastricht and surroundings by advertisements in local newspapers. In addition, subjects who had participated in earlier studies at our Department were approached. People, who were willing to participate, were given a detailed description of the study. After signing the informed consent form, the volunteers were invited for two screening visits (with an interval of $\geq 2$ days). A screening visit consisted of measurements of body weight, height, blood pressure, serum total and HDL cholesterol concentrations, serum triacylglycerol (TAG) concentrations, presence of glucosuria, and hematological parameters. In addition, all subjects completed a general and a medical questionnaire. Forty-three men and women were selected for the study according to the following inclusion criteria: stable body weight (weight gain or loss $<3 \mathrm{~kg}$ in the past 3 months); Quetelet-index $<32 \mathrm{~kg} / \mathrm{m}^{2}$; systolic blood pressure $<160 \mathrm{mmHg}$ and diastolic blood pressure $<95 \mathrm{mmHg}$; mean serum total cholesterol concentrations between $5.0 \mathrm{mmol} / \mathrm{L}$ and $8.0 \mathrm{mmol} / \mathrm{L}$; mean serum TAG concentrations $<4.0 \mathrm{mmol} / \mathrm{L}$; no presence of glucosuria, proteinuria or anemia; no use of medication or a prescribed diet known to affect lipid or glucose metabolism; no history of CHD, cancer, diabetes, kidney, liver, pancreatic disease or malignancies $<5$ y ago; no abuse of drugs and/or alcohol; no pregnant or breast-feeding women; willingness to stop the consumption of vitamin supplements, fish oil capsules or products rich in plant sterols or stanols 3 weeks before the start of the study. Blood donation or participation in another biomedical trial was not allowed 30 days before and during the study. The Medical Ethical Committee of the University of Maastricht had approved the study and all subjects gave written informed consent. 
One subject withdrew in the second week of the first period of the study because he started blood pressure medication. All other 42 volunteers, 20 men and 22 women, completed the study. The mean $( \pm \mathrm{SD})$ age of the men was $54 \pm 10 \mathrm{y}$, and mean Quetelet-index $26 \pm 2$ $\mathrm{kg} / \mathrm{m}^{2}$. For the women, these values were $51 \pm 12$ y and $24 \pm 3 \mathrm{~kg} / \mathrm{m}^{2}$, respectively. Fasting concentrations of serum total, LDL, HDL cholesterol, and TAG in men were $6.66 \pm 0.82$ $\mathrm{mmol} / \mathrm{L}, 4.48 \pm 0.77 \mathrm{mmol} / \mathrm{L}, 1.41 \pm 0.35 \mathrm{mmol} / \mathrm{L}$, and $1.69 \pm 0.81 \mathrm{mmol} / \mathrm{L}$, respectively. In women, these values were $6.59 \pm 0.89 \mathrm{mmol} / \mathrm{L}, 4.10 \pm 0.84 \mathrm{mmol} / \mathrm{L}, 1.83 \pm 0.42$ $\mathrm{mmol} / \mathrm{L}$, and $1.45 \pm 0.69 \mathrm{mmol} / \mathrm{L}$, respectively.

\section{Study design}

The study had a randomized, double-blinded, controlled, multiple-crossover design. Before the study started, the subjects were randomly divided into 3 diet groups. During the first 4 weeks, one group consumed muesli enriched with wheat fiber (control muesli), a second group consumed a muesli enriched with $\beta$-glucan from oats (oat $\beta$-glucan enriched muesli), and a third group a muesli enriched with both oat $\beta$-glucan and plant stanols (combination muesli). After a washout period of 2 weeks, during which time the participants consumed their habitual diets, subjects crossed over to another type of muesli for another period of 4 weeks. After this period, a second washout period of 2 weeks was introduced. For the last 4 weeks of the study, subjects received the type of muesli that they had not consumed during the first two periods. Subjects consumed the products twice daily (2 sachets of $50 \mathrm{~g}$ ), with an interval of at least $5 \mathrm{~h}$.

To improve swelling of the fibers, the muesli had to be consumed with $200 \mathrm{~mL}$ liquid (e.g. milk, orange juice) or with yogurt. To avoid weight gain, subjects were urged to consume the muesli instead of another food item (e.g. bread, cereals). The energy content (1700 kJ/ $100 \mathrm{~g})$, total fiber content, fat/protein/carbohydrate content, and fatty acid composition of the three experimental products were the same. Each sachet of the oat $\beta$-glucan muesli provided $2.5 \mathrm{~g} \beta$-glucan from oats. A sachet of the combination muesli also provided $2.5 \mathrm{~g}$ oat $\beta$-glucan with $0.75 \mathrm{~g}$ plant stanols, provided as fatty acid esters. Extra daily consumption of oat $\beta$-glucan was therefore 0 or $5 \mathrm{~g}$, and daily consumption of plant stanols $1.5 \mathrm{~g}$ (Table 1). 
TABLE 1: Composition of the experimental products'.

\begin{tabular}{lccc}
\hline & Control & Oat $\beta$-glucan & Combination \\
\hline Energy $(\mathrm{kJ})$ & 1683 & 1671 & 1672 \\
Protein $(\mathrm{g})$ & 9.60 & 13.40 & 12.40 \\
Carbohydrates $(\mathrm{g})$ & 13.90 & 14.20 & 15.10 \\
Lipids (g) & 15.20 & 15.30 & 14.60 \\
Total fiber (g) & 11.40 & 11.70 & 11.50 \\
$\beta$-Glucan (g) & 0.00 & 5.09 & 5.03 \\
Stanols (g) & 0.00 & 0.00 & $1.48^{1}$ \\
\hline
\end{tabular}

${ }^{1}$ Plus $0.10 \mathrm{~g}$ sterols

Body weight without shoes or heavy clothes was recorded on day 0 and at the end of weeks 3 and 4 . At the end of the treatment period, subjects recorded their food intake (previous 3 weeks) by completing food-frequency questionnaires (FFQ) to estimate their energy and nutrient intakes. FFQ were checked by a registered dietician in the presence of the subjects. The subjects recorded any illness, medication used, menstrual phase, alcohol consumption, any deviations to the study protocol, and any side-effects experienced (headache, stomach complaints, nausea, bloated feeling, flatulence, diarrhea, constipation, itching, eruptions/rashes, fatigue, and dizziness).

Other possible side-effects were monitored on day 0 and at the end of week 4 by assessing hematological parameters and parameters to measure kidney and liver function.

\section{Blood sampling}

Volunteers fasted overnight, did not use alcohol the previous day and did not smoke on the morning of blood sampling. Venous blood samples were taken between 07:45 and 11:00 on day 0 and on weeks 3 and 4 of each study period. All venipunctures were generally done by the same person, in the same room, and at the same time of the day. Blood was sampled from a forearm vein using vacutainers under minimal stasis with the subject in supine position.

Ten $\mathrm{mL}$ of blood was collected in a serum tube (Becton Dickinson Vacutainer Systems, Franklin Lakes, NJ) for analysis of serum lipids (total serum cholesterol, HDL cholesterol, total TAG), lipoproteins (apoB and apoA-I), plant sterols and stanols (sitosterol, sitostanol, campesterol, and campestanol), a cholesterol precursor (lathosterol), and indices of kidney and liver function. Serum tubes were kept at room temperature after blood sampling. At least $1 \mathrm{~h}$ after venipuncture, serum was obtained by centrifuging at $2000 \times \mathrm{g}$ for $30 \mathrm{~min}$ at $4^{\circ} \mathrm{C}$. Serum was aliquoted and thereafter stored at $-80^{\circ} \mathrm{C}$. Furthermore, $10 \mathrm{~mL}$ of blood was collected in an EDTA tube (Becton Dickinson Vacutainer Systems) for analysis of hematological variables, lipid-soluble antioxidants, and $7 \alpha$-hydroxy-4-cholesten-3-one ( $7 \alpha$-OHC), 
a marker of bile acid synthesis. Hematological parameters were determined directly after sampling. Plasma was obtained immediately by centrifugation at $2000 \times \mathrm{g}$ for $30 \mathrm{~min}$ at $4^{\circ} \mathrm{C}$. Plasma was then divided into aliquots, snap-frozen, and stored at $-80^{\circ} \mathrm{C}$.

Analyses

Concentrations of total cholesterol (ABX Diagnostics, Montpellier, France), HDL cholesterol (precipitation method; Roche Diagnostics Corporation, Indianapolis, IN), and TAG corrected for free glycerol (Sigma-Aldrich Chemie, Steinheim, Germany) were analyzed enzymatically in serum. ApoA-I and apoB concentrations were analyzed in serum using an immunoturbidimetric method (ABX Diagnostics, Montpellier, France). Serum LDL cholesterol concentrations were calculated by using the formula of Friedewald et al. (112). All samples from one subject were analyzed in the same analytical run.

Serum plant sterols and stanols (sitosterol, sitostanol, campesterol, and campestanol) and the cholesterol precursor lathosterol were determined as described (111). Before analysis, serum samples of weeks 3 and 4 of each period were pooled.

Serum samples were also analyzed to determine parameters of liver function (alanine transaminase, aspartate aminotransferase, $\gamma$-glutamyl transpeptidase (GGT), alkaline phosphatase, and total bilirubin), kidney function (creatinine), and inflammation (Creactive protein; CRP). These measurements were carried out on a Beckman Coulter Synchron LX20 PRO Clinical System (Beckman Coulter Inc., Fullerton, CA).

Plasma samples were analyzed on a Coulter Ac• $\bullet$ diff (Coulter Corporation, Miami, FL) for the following hematological variables: number of white blood cells, $\%$ of lymphocytes, $\%$ of monocytes, \% of granulocytes, number of red blood cells, hemoglobin, hematocrit, mean corpuscular volume, and number of platelets.

Before analyzing lipid-soluble antioxidants, plasma samples taken at weeks 3 and 4 of each period were pooled. Concentrations of tocopherols $(\alpha$-tocopherol, $\delta$-tocopherol, and $\beta$ and $\gamma$-tocopherol), carotenoids ( $\alpha$-carotene, $\beta$-carotene, lycopene, lutein/zeaxanthin, $\beta$ cryptoxanthin, and phytofluene), and retinol were determined simultaneously according to a modification of the assay of Hess et al (113), as described by Oostenbrug et al. (114). All samples from one subject were analyzed in the same analytical run.

Before analyzing $7 \alpha-\mathrm{OHC}$, plasma samples of weeks 3 and 4 of each period were pooled. $7 \alpha-\mathrm{OHC}$ was determined by HPLC after modification of the method described by Gälman et al. (115). All analyses were done in duplicate. Briefly, plasma $(0.5 \mathrm{~mL})$ was diluted with $2.5 \mathrm{~mL}$ of saline and $25 \mu \mathrm{L}$ of the internal standard [7 $\beta$-hydroxy-4-cholesten-3-one (5 $\mathrm{ng} / \mu \mathrm{L}$ ) dissolved in $\mathrm{H}_{2} \mathrm{O}$ ] was added. EC-C8 Isolute SPE columns $(500 \mathrm{mg}, 3 \mathrm{~mL}$, Sopachem $\mathrm{NV}$, Brussels, Belgium) were prewashed with $2 \times 2 \mathrm{~mL}$ pure methanol and with $2 \times 2 \mathrm{~mL}$ of water. Columns were then mounted on a Vac Elut SPS 24 (Analytichem International, 
Harbor City, CA), to which a removable custom-made stainless steel water-jacketed block heater with 12 positions had been added. The column heater was connected to a water bath (model K6, MGW Lauda, Königshofen, Germany) set on $65.3^{\circ} \mathrm{C}$ in order to obtain a column temperature of $64^{\circ} \mathrm{C}$. To facilitate passage through the columns, the samples were first heated to $64^{\circ} \mathrm{C}$ for $20 \mathrm{~min}$. Subsequently, the supernatant, obtained after centrifugation at $2000 \times \mathrm{g}$ for $5 \mathrm{~min}$ at $2{ }^{\circ} \mathrm{C}$, was sonicated in water for $15 \mathrm{~min}$ and incubated at $37^{\circ} \mathrm{C}$ for 15 min prior to loading onto the preheated columns. A stream of nitrogen was used occasionally to facilitate passage through columns. The eluted product was evaporated to dryness at $60^{\circ} \mathrm{C}$ under a stream of nitrogen and dissolved in $110 \mu \mathrm{L}$ acetonitrile; $75 \mu \mathrm{L}$ of this was injected into the HPLC system. All samples from one subject were analyzed within one run. $7 \alpha-\mathrm{OHC}$ was quantified from the heights of the respective peaks using the known amount of internal standard and the response factor.

\section{Statistical analyses}

The statistical power needed to detect a true difference of $5 \%$ in serum LDL concentrations between the treatments with $\alpha=0.05$ was $80 \%$. Before the statistical analyses were carried out, serum lipid and lipoprotein concentrations from weeks 3 and 4 of each period were averaged for each subject. The data were analyzed by a three-way (subject number, period, and diet) ANOVA using the general linear model procedure. Differences in effects on the variables of interest were examined with diet and period as fixed factors, and subject number as a random factor. When a significant diet effect was found, the 3 treatments were compared pairwise using a Tukey post-hoc test for multiple comparisons. Values are presented as means \pm SD. Differences were considered significant at $p \leq 0.05$. Statistical analyses were performed using SPSS 11.0 (version 11.0.3) for Maclntosh OS X (version 10.3.9).

\section{RESULTS}

Side effects

Side effects were monitored by assessing hematological parameters, and indices of kidney and liver function. Throughout the study, all variables remained within the normal range for all subjects and no treatment effects were present. Compared to other subjects, however, GGT values were consistently increased for two subjects during the study (100 $\mathrm{U} / \mathrm{L}$ and $180 \mathrm{U} / \mathrm{L}$ during the control period, $90 \mathrm{U} / \mathrm{L}$ and $132 \mathrm{U} / \mathrm{L}$ during the oat $\beta$-glucan 
period, and $93 \mathrm{U} / \mathrm{L}$ and $105 \mathrm{U} / \mathrm{L}$ during the combination period). Results of these two subjects were therefore excluded from further statistical analyses. Except for the effects on the marker of bile acid synthesis $7 \alpha-\mathrm{OHC}$, this did not change the conclusions.

\section{Dietary intake and body weight}

As calculated from the returned sachets, daily consumption of oat $\beta$-glucan during the oat $\beta$-glucan period was $4.8 \pm 0.3$ (mean \pm SD) g. During the combination period, daily intake of oat $\beta$-glucan and plant stanols was $4.8 \pm 0.3 \mathrm{~g}$ and $1.4 \pm 0.1 \mathrm{~g}$, respectively.

Daily energy intake and nutrient composition of the diets did not differ among the 3 treatment groups, except for polyunsaturated fatty acids ( $p=0.045$ for diet effect). However, between-diet comparisons were not significant (Table 2).

TABLE 2: Nutrient composition of the 3 diets according to the food-frequency questionnaires ${ }^{1}$.

\begin{tabular}{|c|c|c|c|c|}
\hline & Control & Oat $\beta$-glucan & Combination & $p$ for diet effect ${ }^{2}$ \\
\hline \multicolumn{5}{|l|}{ Energy } \\
\hline$(\mathrm{MJ} / \mathrm{d})$ & $8.1 \pm 2.4$ & $8.3 \pm 2.5$ & $8.0 \pm 2.4$ & 0.256 \\
\hline$(\mathrm{kcal} / \mathrm{d})$ & $1929 \pm 582$ & $1980 \pm 603$ & $1919 \pm 581$ & 0.255 \\
\hline Protein (\% of energy) & $17.8 \pm 3.0$ & $18.0 \pm 3.2$ & $18.0 \pm 3.4$ & 0.815 \\
\hline Fat (\% of energy) & $34.0 \pm 5.7$ & $34.0 \pm 5.5$ & $33.7 \pm 5.7$ & 0.737 \\
\hline Saturated fatty acids & $12.5 \pm 2.8$ & $12.2 \pm 2.4$ & $12.4 \pm 2.5$ & 0.450 \\
\hline Monounsaturated fatty acids & $11.1 \pm 2.6$ & $11.1 \pm 2.7$ & $11.0 \pm 2.6$ & 0.990 \\
\hline Polyunsaturated fatty acids & $6.5 \pm 6.6$ & $6.8 \pm 1.7$ & $6.4 \pm 1.6$ & 0.045 \\
\hline Carbohydrates ( $\%$ of energy) & $45.7 \pm 5.8$ & $45.1 \pm 5.3$ & $45.8 \pm 6.1$ & 0.425 \\
\hline Alcohol (\% of energy) & $2.7 \pm 2.6$ & $3.0 \pm 2.8$ & $2.8 \pm 2.9$ & 0.506 \\
\hline Cholesterol (mg/MJ) & $23.3 \pm 6.1$ & $22.2 \pm 5.3$ & $22.1 \pm 6.5$ & 0.139 \\
\hline Dietary fiber (g/MJ) & $2.8 \pm 0.8$ & $2.7 \pm 0.7$ & $2.7 \pm 0.8$ & 0.457 \\
\hline
\end{tabular}

${ }^{1}$ All values are means \pm SD; $n=40$ (19 men and 21 women). Pairwise comparisons by the least-squares means procedure with Tukey's adjustment in a general linear model did not reveal any significant differences between any two diets.

${ }^{2}$ Calculated by using a general linear model with subject number as a random factor and diet and period as fixed factors.

Body weights did not differ after the control $(74 \pm 11 \mathrm{~kg})$, oat $\beta$-glucan $(74 \pm 11 \mathrm{~kg})$, and combination $(74 \pm 11 \mathrm{~kg})$ diet periods. Weight changes during each diet period were significant, but consistent $[0.42 \pm 0.80 \mathrm{~kg}$ during the control period $(p=0.001), 0.37 \pm 0.93$ $\mathrm{kg}$ during the oat $\beta$-glucan period ( $\mathrm{p}=0.013$ ), and $0.25 \pm 0.68 \mathrm{~kg}$ during the combination period $(p=0.027)]$. 


\section{Serum lipids and lipoproteins}

The oat $\beta$-glucan enriched muesli decreased serum total cholesterol concentrations by 0.23 $\mathrm{mmol} / \mathrm{L}(3.4 \%)$ compared to the control muesli $(\mathrm{p}=0.015)$ (Table 3). The combination muesli reduced total cholesterol concentrations by $0.48 \mathrm{mmol} / \mathrm{L}(7.1 \%)$ compared to the control muesli $(\mathrm{p}<0.001)$, and by $0.25 \mathrm{mmol} / \mathrm{L}(3.6 \%)$ compared to the oat $\beta$-glucan enriched muesli $(p=0.008)$.

TABLE 3: Effects of 4-weeks daily intake of oat $\beta$-glucan and oat $\beta$-glucan plus plant stanols on fasting serum lipid and lipoprotein concentrations in healthy men and women'.

\begin{tabular}{lcccc}
\hline & Control & Oat $\beta$-glucan & Combination & $\mathrm{p} \mathrm{for} \mathrm{diet} \mathrm{effect}^{2}$ \\
\hline Total cholesterol $(\mathrm{mmol} / \mathrm{L})$ & $6.49 \pm 1.03$ & $6.26 \pm 0.96^{\mathrm{a}}$ & $6.01 \pm 0.92^{\mathrm{a}, \mathrm{b}}$ & $<0.001$ \\
LDL cholesterol $(\mathrm{mmol} / \mathrm{L})$ & $4.31 \pm 0.96$ & $4.09 \pm 0.91^{\mathrm{a}}$ & $3.89 \pm 0.90^{\mathrm{a}, \mathrm{b}}$ & $<0.001$ \\
HDL cholesterol $(\mathrm{mmol} / \mathrm{L})$ & $1.46 \pm 0.46$ & $1.45 \pm 0.49$ & $1.45 \pm 0.52$ & 0.768 \\
TAG $(\mathrm{mmol} / \mathrm{L})$ & $1.52 \pm 0.84$ & $1.66 \pm 1.36$ & $1.56 \pm 0.94$ & 0.277 \\
Total: $\mathrm{HDL}$ cholesterol & $5.03 \pm 1.12$ & $4.81 \pm 1.09^{\mathrm{a}}$ & $4.56 \pm 1.01^{\mathrm{a}, \mathrm{b}}$ & $<0.001$ \\
ApoB-100 $(\mathrm{g} / \mathrm{L})$ & $1.24 \pm 0.27$ & $1.21 \pm 0.27$ & $1.15 \pm 0.25^{\mathrm{a}, \mathrm{b}}$ & $<0.001$ \\
ApoA-I $(\mathrm{g} / \mathrm{L})$ & $1.62 \pm 0.31$ & $1.60 \pm 0.34$ & $1.57 \pm 0.32$ & 0.065 \\
\hline
\end{tabular}

${ }^{1}$ All values are means $\pm S D ; n=40$ (19 men and 21 women). Superscript letters indicate significant differences $(\mathrm{p} \leq 0.05)$ : avs. control; 'bs. oat $\beta$-glucan (pairwise comparisons by least-squares means procedure with Tukey's adjustment in a general linear model).

${ }^{2}$ Calculated by using a general linear model with subject number as a random factor and diet and period as fixed factors.

After oat $\beta$-glucan consumption, serum LDL cholesterol concentrations were $0.21 \mathrm{mmol} / \mathrm{L}$ $(5.0 \%)$ lower than after the control period $(\mathrm{p}=0.013)$. The combination muesli effectively lowered LDL cholesterol concentrations by $0.39 \mathrm{mmol} / \mathrm{L}(9.6 \%)$ compared to the control muesli $(\mathrm{p}<0.001)$, and by $0.18 \mathrm{mmol} / \mathrm{L}(4.4 \%)$ compared to oat $\beta$-glucan intake only $(\mathrm{p}=0.036)$.

Consumption of oat $\beta$-glucan significantly decreased the ratio of total-to-HDL cholesterol by $0.09(1.7 \%)$ compared to the control muesli $(p=0.016)$. Consumption of oat $\beta$-glucan plus plant stanols reduced the ratio of total-to-HDL cholesterol by 0.27 (5.5\%) compared to the control muesli $(p<0.001)$, and by $0.18(3.4 \%)$ compared to consumption of oat $\beta$ glucan only $(\mathrm{p}=0.004)$.

The combination muesli lowered serum apoB concentrations by $0.09 \mathrm{~g} / \mathrm{L}(6.7 \%)$ compared to the control muesli $(\mathrm{p}<0.001)$, and by $0.06 \mathrm{~g} / \mathrm{L}(4.1 \%)$ compared to the oat $\beta$-glucan enriched muesli $(p=0.003)$. ApoB concentrations did not differ after the control and oat $\beta$ glucan periods. Serum HDL cholesterol, apoA-I, and TAG concentrations did not differ after the 3 diet treatments. 
Markers for cholesterol absorption, cholesterol synthesis, and bile acid synthesis

Oat $\beta$-glucan consumption increased cholesterol-standardized serum lathosterol concentrations by $0.20 \mu \mathrm{mol} / \mathrm{mmol}(17.6 \%$ ) compared to the control muesli ( $\mathrm{p}=0.050)$, indicating an elevated cholesterol synthesis (Table 4). The combination muesli increased cholesterolstandardized serum lathosterol concentrations by $0.26 \mu \mathrm{mol} / \mathrm{mmol}(19.1 \%)$ compared to the control muesli $(p=0.016)$. Relative to the oat $\beta$-glucan enriched muesli, the combination muesli did not alter cholesterol-standardized serum lathosterol concentrations $(p=0.814)$.

TABLE 4: Effects of 4-weeks daily intake of oat $\beta$-glucan and oat $\beta$-glucan plus plant stanols on markers for cholesterol synthesis, cholesterol absorption, and bile acid synthesis after correction for total cholesterol in healthy men and women'.

\begin{tabular}{llcrr}
\hline & Control & Oat $\beta$-glucan & Combination & p for diet effect ${ }^{2}$ \\
\hline Lathosterol & $195 \pm 60$ & $215 \pm 60^{\mathrm{a}}$ & $221 \pm 57^{\mathrm{a}}$ & 0.010 \\
Campesterol & $318 \pm 145$ & $282 \pm 129^{\mathrm{a}}$ & $241 \pm 114^{\mathrm{a}, \mathrm{b}}$ & $<0.001$ \\
Sitosterol & $123 \pm 60$ & $113 \pm 55$ & $90.7 \pm 44^{\mathrm{a}, \mathrm{b}}$ & $<0.001$ \\
Campestanol & $8.26 \pm 7.19$ & $6.72 \pm 4.77$ & $6.69 \pm 6.36$ & 0.164 \\
Sitostanol & $2.26 \pm 1.19$ & $2.10 \pm 1.21$ & $1.62 \pm 0.94^{\mathrm{a}}$ & 0.008 \\
$7 \alpha$-OHC & $3.57 \pm 2.22$ & $4.28 \pm 3.00^{\mathrm{a}}$ & $4.18 \pm 3.03$ & 0.031 \\
\hline
\end{tabular}

${ }^{1}$ All values are means $\pm S D ; n=40$ (19 men and 21 women), except for analyses of sterols and stanols $(\mathrm{n}=39 ; 19$ men and 20 women; 1 missing value). All values are expressed in $10^{2} \times \mu \mathrm{mol} / \mathrm{mmol}$ total cholesterol, except for $7 \alpha-\mathrm{OHC}$

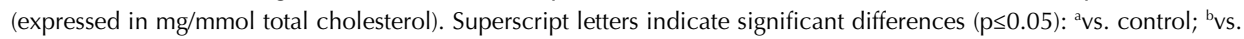
oat $\beta$-glucan (pairwise comparisons by least-squares means procedure with Tukey's adjustment in a general linear model)

${ }^{2}$ Calculated by using a general linear model with subject number as a random factor and diet and period as fixed factors.

Compared to the control period, cholesterol-standardized serum campesterol concentrations significantly decreased by $0.36 \mu \mathrm{mol} / \mathrm{mmol}(7.1 \%)$ after oat $\beta$-glucan consumption $(p=0.011)$, indicating decreased intestinal cholesterol absorption. The muesli enriched with oat $\beta$-glucan plus plant stanols significantly decreased cholesterol-standardized campesterol concentrations by $0.76 \mu \mathrm{mol} / \mathrm{mmol}(20.7 \%)$ compared to the control muesli $(\mathrm{p}<0.001)$, and by $0.40 \mu \mathrm{mol} / \mathrm{mmol}(11.1 \%)$ compared to oat $\beta$-glucan intake only $(p=0.004)$. Changes in sitosterol concentrations paralleled those of campesterol, except that the effects of the oat $\beta$-glucan enriched muesli did not differ from those of the control muesli.

The combination muesli significantly decreased cholesterol-standardized serum sitostanol concentrations by $0.007 \mu \mathrm{mol} / \mathrm{mmol}(21.3 \%)$ compared to the control muesli $(p=0.010)$. Compared to oat $\beta$-glucan intake only, the effects of the combined intake on cholesterol- 
standardized serum sitostanol concentrations almost reached significance $(p=0.066)$. The control and oat $\beta$-glucan periods did not differ in cholesterol-standardized serum sitostanol concentrations $(p=0.753)$. Campestanol concentrations did not differ significantly between the 3 treatment groups.

Compared to the control muesli, the muesli enriched with oat $\beta$-glucan effectively increased cholesterol-standardized plasma $7 \alpha-\mathrm{OHC}$ concentrations by $0.70 \mathrm{mg} / \mathrm{mmol}$ $(24.4 \%)(p=0.043)$. The control and combination, and the oat $\beta$-glucan and combination periods did not differ in cholesterol-standardized plasma $7 \alpha-\mathrm{OHC}$ concentrations $(p=0.106$ and $\mathrm{p}=1.000$, respectively).

Lipid-soluble antioxidants

After correction for serum LDL cholesterol concentrations, plasma total tocopherol, oxygenated carotenoid, and hydrocarbon carotenoid concentrations did not differ significantly between the 3 treatment periods. Also, plasma retinol concentrations were not affected (data not shown).

\section{DISCUSSION}

In this study, consumption of muesli enriched with oat $\beta$-glucan significantly decreased serum LDL cholesterol concentrations. Subjects consumed twice daily $2.4 \mathrm{~g}$ of oat $\beta$ glucan, while the observed decrease in serum LDL cholesterol was $0.21 \mathrm{mmol} / \mathrm{L}$. This is in agreement with the predicted reduction in LDL cholesterol of $0.18 \mathrm{mmol} / \mathrm{L}$, based on the meta-analysis by Brown et al. (59). The reported study results on the hypocholesterolemic effects of oat $\beta$-glucan are however variable. Inconsistencies in the reported effects of oat products may be due to several factors, such as mode of administration, or differences in solubility or molecular weight.

The combined effects of oat $\beta$-glucan and plant stanols on LDL cholesterol concentrations have not been systematically examined. We found that addition of plant stanols to the oat $\beta$-glucan enriched muesli significantly lowered LDL cholesterol concentrations by $4.4 \%$. This effect is smaller than the estimated mean change of $-8.5 \%$ for daily intakes of 1.5 to $1.9 \mathrm{~g}$ sterols or stanols (14). Thus, our findings suggest that the viscous soluble fiber $\beta$ glucan from oats may reduce the efficacy of plant stanols. However, several studies have concluded that the effects of plant sterols and stanols do not change when part of a recommended diet $(14,116)$, which is usually accompanied by a decrease in fat and cholesterol intake. When fats are replaced by complex carbohydrates, an increase in fiber intake can be expected as well. The majority of these studies (117-122) did unfortunately 
not document (changes in) the intake of viscous soluble fibers. Recently, Yoshida et al. (123) examined the effects of $1.8 \mathrm{~g} / \mathrm{d}$ free plant sterols with or without $10 \mathrm{~g} / \mathrm{d}$ glucomannan, a viscous soluble fiber derived from konjac root, in individuals with and without type II diabetes. In the non-diabetic group, serum LDL cholesterol concentrations decreased after the plant sterol, glucomannan, and combined intakes by $5.4 \%, 13.8 \%$, and $21.7 \%$, respectively. The results were less pronounced in the diabetic patients. Although the effects of the plant sterols were less than predicted, these findings did not suggest that glucomannan attenuated the LDL-cholesterol lowering effects of the free plant sterols. In another recent study, a combination therapy including $2.6 \mathrm{~g} / \mathrm{d}$ plant sterols and $10 \mathrm{~g} / \mathrm{d}$ psyllium also decreased LDL cholesterol beyond expectations (14, 59). According to the literature, daily consumption of $>2.5$ g sterols or stanols lowers LDL cholesterol concentrations on average by $11.3 \%$, which is already higher than the $10 \%$ decrease in serum LDL cholesterol levels found after the combination therapy. However, separate contribution of the individual components was not evaluated (124). Also other recent studies with similar intakes of plant sterols and stanols have reported effects less than the estimated mean change of $-8.5 \%$ (125-128). Moreover, Clifton et al. (129) reported that plant sterols (1.6 $\mathrm{g} / \mathrm{d}$ ) incorporated in milk, yoghurt, bread, and cereals decreased serum LDL cholesterol concentrations by $15.9 \%, 8.6 \%, 6.5 \%$, and $5.4 \%$, respectively. Their results suggest that the food matrix may influence the cholesterol-lowering effectiveness of plant sterols. Altogether, the estimated mean percentage reduction of $8.5 \%$ in LDL cholesterol at daily intakes of 1.5 to $2.1 \mathrm{~g}$ of plant sterols or stanols may be overrated (14). Nevertheless, the $4.4 \%$ change in LDL cholesterol is somewhat less than in other studies at comparable intakes and an effect of oat $\beta$-glucan or the food matrix on the efficacy of plant stanols can therefore not be excluded. In support, oat $\beta$-glucan consumption decreased serum cholesterol-standardized stanol concentrations, although the effect on campestanol was not statistically significant $(p=0.164)$. Also adding plant stanols to the oat $\beta$-glucan enriched muesli did not change serum cholesterol-standardized stanol concentrations. Consumption of plant stanols normally leads to an increase in their serum concentrations (64). Thus, these findings suggest that oat $\beta$-glucan may interfere with the absorption of plant stanols. Plant stanols reduce intestinal cholesterol absorption through competition for micellar incorporation (110). However, recent findings indicate that stanols may also enter the intestinal cells and promote cholesterol efflux back into the intestinal lumen $(18,110)$. We speculate that a higher intestinal viscosity may lower the transport of plant stanols into the enterocytes. As a result, not only their serum concentrations, but also their cholesterollowering efficacy may decrease. Alternatively, an increase in intestinal viscosity may affect lipid emulsification by increasing the droplet size of emulsions thereby decreasing the emulsion interface area. This may reduce the rate of lipolysis (130) and possibly also the hydrolysis of plant stanols. It can further be speculated that the active free plant stanols are released later in the intestinal tract, which may affect their efficacy and absorption. 
Oat $\beta$-glucan consumption significantly increased total cholesterol-standardized $7 \alpha$-OHC concentrations, indicating an increased bile acid synthesis. Additionally, cholesterolstandardized campesterol and sitosterol concentrations decreased, suggesting reduced cholesterol absorption, although only the effect on campesterol was statistically significant. Cholesterol-standardized lathosterol concentrations, which are positively related with endogenous cholesterol synthesis, increased. Overall, these findings are in line with the proposed mechanism by which viscous soluble fibers decrease serum cholesterol concentrations $(64,68,92,108)$. Addition of plant stanols to the muesli enriched with oat $\beta$ glucan did not further increase bile acid synthesis. In addition, plant stanols significantly lowered cholesterol absorption and tended to increase endogenous cholesterol synthesis, in line with their suggested working mechanism.

Since viscous soluble fibers can change the physical characteristics of the contents of the small intestine, they may interfere with the absorption of lipid-soluble antioxidants. Oat $\beta$ glucan consumption did, however, not change LDL cholesterol-standardized lipid-soluble antioxidant concentrations, which agrees with the results of previous studies $(64,131)$. In addition, we found no effects of a daily intake of $1.4 \mathrm{~g}$ plant stanols on LDL cholesterolstandardized lipid-soluble antioxidant concentrations. Indeed, a meta-analysis showed that lipid-soluble antioxidants were not affected by stanols after correction for the decrease in cholesterol, with the exception of $\beta$-carotene (14). However, effects on $\beta$-carotene concentrations were mainly seen at daily intakes of $>2$ g plant stanols $(14,18)$.

In summary, our results showed that muesli enriched with oat $\beta$-glucan effectively lowered serum LDL cholesterol concentrations. Addition of plant stanols to the oat $\beta$-glucan enriched muesli further lowered serum LDL cholesterol levels, although less than predicted. Whether this is due to an effect of the viscous soluble fiber oat $\beta$-glucan or the food matrix needs further investigation.

\section{ACKNOWLEDGEMENTS}

We thank the study participants for their cooperation and enthusiasm. We also thank the technical and dietary staff from our Department for their support. 


\section{CHAPTER 4}

\section{Oat $\beta$-glucan with or without plant stanols did not influence inflammatory markers in hypercholesterolemic subjects}

Elke Theuwissen, Jogchum Plat, Ronald P. Mensink

Based on:

Mol Nutr Food Res. 2008; In press 


\section{ABSTRACT}

Objective We have earlier demonstrated that muesli enriched with oat $\beta$-glucan effectively lowered serum LDL cholesterol concentrations. Addition of plant stanols further lowered serum LDL cholesterol levels. Besides these hypocholesterolemic effects, oat $\beta$-glucan and plant stanols may also affect inflammatory processes.

Study design Forty-two mildly hypercholesterolemic subjects randomly received for 4 weeks in a crossover design: control muesli ( $5 \mathrm{~g} / \mathrm{d}$ of control fiber), oat $\beta$-glucan enriched muesli ( $5 \mathrm{~g} / \mathrm{d}$ of oat $\beta$-glucan), or combination muesli $(5 \mathrm{~g} / \mathrm{d}$ of oat $\beta$-glucan plus $1.5 \mathrm{~g} / \mathrm{d}$ of plant stanols). Changes in cytokine production (IL-6, IL-8, and TNF- $\alpha$ ) of LPS-stimulated peripheral blood mononuclear cells (PBMC) and whole blood were evaluated, as well as changes in plasma high-sensitivity (hs)-CRP. Additionally, changes in expression profiles of 84 genes involved in atherosclerosis metabolism were assessed in isolated PBMC.

Results IL-6, IL-8, and TNF- $\alpha$ production by PBMC and whole blood after LPS stimulation did not differ between the treatments. Also hs-CRP levels were similar. Oat $\beta$-glucan consumption did not change gene expression, while only 3 genes (ADFP, CDH5, CSF2) out of the 84 genes from the atherosclerotic risk panel were differentially expressed $(p<0.05)$ after consumption of plant stanols.

Conclusion Consumption of oat $\beta$-glucan with or without plant stanols did not influence inflammatory parameters in mildly hypercholesterolemic subjects. 


\section{INTRODUCTION}

It is becoming more and more established that inflammation plays an important role in the development of atherosclerosis and cardiovascular disease (CVD). Many studies have therefore been initiated to examine the effects of diets and dietary components on inflammatory markers. In this respect, much attention is paid to functional foods. Such foods contain (or lack) one or more components and therefore provide positive health effects beyond their traditional nutritional value. Examples of such food components with approved FDA-health claims are the viscous soluble fiber $\beta$-glucan from oats (10), and plant sterols and stanols (12). These components were approved because of their consistent LDL cholesterol-lowering effects. We have recently demonstrated that muesli enriched with $\beta$-glucan derived from oats effectively lowered serum LDL cholesterol concentrations in slightly hypercholesterolemic subjects. Addition of plant stanols to the oat $\beta$-glucan enriched muesli even further lowered serum LDL cholesterol levels (63).

Besides these hypocholesterolemic effects, in vitro and animal studies have suggested that oat $\beta$-glucan and phytosterols may also affect immune and inflammatory processes (29-37). Data from human studies are however limited and inconsistent. So far, only one study has examined the effects of oat $\beta$-glucan on inflammatory parameters. In this study, Queenan et al. could not demonstrate that dietary supplementation with oat $\beta$-glucan changed plasma concentrations of C-reactive protein (CRP) in hypercholesterolemic subjects (61). Like for oat $\beta$-glucan, effects of plant sterol and stanols on inflammatory markers have been hardly studied. Several studies showed no significant effects on markers of inflammation, such as CRP, soluble adhesion molecules, and monocyte chemotactic protein-1 (MCP1) (132-134). Nevertheless, Cater et al. (135) showed that combined administration of plant stanols with a statin compared to statin-treatment alone significantly reduced CRP concentrations in patients with a positive history for CVD. On the other hand, they found no significant change in CRP levels of hypercholesterolemic subjects with plant stanols alone. Devaraj et al. (136) found a significant reduction in CRP levels after consumption of a sterol-enriched orange juice in healthy subjects. Altogether, more research is needed to clarify the role of these cholesterol-lowering dietary components on the inflammatory processes related to atherosclerosis and CVD.

To explore whether the effects of oat $\beta$-glucan enriched muesli with or without plant stanols not only changed LDL cholesterol, but also inflammatory processes, we first examined their effects on plasma high-sensitivity CRP (hs-CRP) concentrations. Hs-CRP is considered a marker for low-grade systemic inflammation and is positively associated with an increased risk for future cardiovascular events (38). Since leukocyte reactivity is easily triggered by modified LDL cholesterol, potential changes may be more evident in hypercholesterolemic subjects. We therefore determined the effects of oat $\beta$-glucan with or without plant stanols on leukocyte function. This was studied ex vivo by measurement of 
inflammatory cytokines (IL-6, IL-8, and TNF- $\alpha$ ) production after LPS stimulation of isolated peripheral blood mononuclear cells (PBMC) and of whole blood. IL-6, IL-8, and TNF- $\alpha$ are pro-inflammatory markers involved in the development of atherosclerotic lesions (137, 138). Finally, we studied in PBMC the effects of these functional ingredients on the expression of 84 genes involved in atherosclerosis.

\section{MATERIALS AND METHODS}

\section{Study population}

This trial was part of a study on the simultaneous effects of oat $\beta$-glucan and plant stanols on the lipoprotein profile in healthy men and women with slightly elevated serum cholesterol concentrations. Details of the study have already been published (63). All participants were given a detailed description of the experimental protocol and purpose of the study before they gave their written informed consent. They were invited for 2 screening visits, which consisted of measurements of body weight, height, blood pressure, serum total and HDL cholesterol concentrations, serum triacylglycerol (TAG) concentrations, presence of glucosuria, and hematological parameters. In addition, all subjects had to fill in a general and medical questionnaire.

Participants were selected for the study according to the following inclusion criteria: stable body weight (weight gain or loss $<3 \mathrm{~kg}$ in the past 3 months); Quetelet-index $<32 \mathrm{~kg} / \mathrm{m}^{2}$; systolic blood pressure $<160 \mathrm{mmHg}$; diastolic blood pressure $<95 \mathrm{mmHg}$; mean serum total cholesterol concentrations between $5.0 \mathrm{mmol} / \mathrm{L}$ and $8.0 \mathrm{mmol} / \mathrm{L}$; mean serum TAG concentrations $<4.0 \mathrm{mmol} / \mathrm{L}$; no presence of glucosuria, proteinuria or anemia; no use of medication or a prescribed diet known to affect lipid or glucose metabolism; no history of coronary heart disease, cancer, diabetes, kidney, liver, pancreatic disease or malignancies $<5$ y ago; no abuse of drugs and/or alcohol; no pregnant or breast-feeding women; willingness to stop the consumption of vitamin supplements, fish oil capsules or products rich in plant stanols or sterols 3 weeks before the start of the study. Blood donation or participation in another biomedical trial was not allowed 30 days before and during the study. The Medical Ethical Committee of the University of Maastricht had approved the study and all subjects gave written informed consent.

Forty-three volunteers were selected for the study. One man withdrew in the $2^{\text {nd }}$ week of the $1^{\text {st }}$ period of the study because he started blood pressure medication. All other 42 volunteers, 20 men and 22 women, completed this study. Baseline characteristics are shown in Table 1. 
TABLE 1: Baseline characteristics of the participants ${ }^{1}$.

\begin{tabular}{|c|c|c|c|c|}
\hline & Control & Oat $\beta$-glucan & Combination & $p$ value ${ }^{2}$ \\
\hline Number (M/F) & $13(8 / 5)$ & $14(6 / 8)$ & $15(6 / 9)$ & \\
\hline Age $(y)$ & $54 \pm 10$ & $51 \pm 13$ & $51 \pm 10$ & 0.769 \\
\hline $\mathrm{BMI}\left(\mathrm{kg} / \mathrm{m}^{2}\right)$ & $25 \pm 3$ & $25 \pm 2$ & $25 \pm 3$ & 0.997 \\
\hline Systolic blood pressure (mmHg) & $139 \pm 17$ & $140 \pm 13$ & $135 \pm 15$ & 0.690 \\
\hline Diastolic blood pressure $(\mathrm{mmHg})$ & $85 \pm 9$ & $88 \pm 8$ & $86 \pm 11$ & 0.723 \\
\hline Total cholesterol $(\mathrm{mmol} / \mathrm{L})$ & $6.53 \pm 0.96$ & $6.53 \pm 1.10$ & $6.14 \pm 1.13$ & 0.537 \\
\hline LDL cholesterol (mmol/L) & $4.02 \pm 1.06$ & $4.43 \pm 1.00$ & $3.88 \pm 0.85$ & 0.307 \\
\hline HDL cholesterol $(\mathrm{mmol} / \mathrm{L})$ & $1.57 \pm 0.59$ & $1.57 \pm 0.42$ & $1.44 \pm 0.49$ & 0.744 \\
\hline TAG $(\mathrm{mmol} / \mathrm{L})$ & $1.90 \pm 1.42$ & $1.15 \pm 0.57$ & $1.63 \pm 0.78$ & 0.140 \\
\hline
\end{tabular}

${ }^{1} \mathrm{All}$ values are presented as means $\pm \mathrm{SD} ; \mathrm{n}=42$.

${ }^{2}$ Data were analyzed by ANOVA using the general linear model procedure. Differences were considered significant at $\mathrm{p}<0.05$.

\section{Study design}

The study had a randomized, double-blinded, controlled, multiple crossover design (63). Briefly, subjects consumed for 4 weeks in random order: control muesli $(4.8 \mathrm{~g} / \mathrm{d}$ of control fiber), oat $\beta$-glucan enriched muesli ( $4.8 \mathrm{~g} / \mathrm{d}$ of oat $\beta$-glucan), or combination muesli [4.8 $\mathrm{g} / \mathrm{d}$ of oat $\beta$-glucan plus $1.4 \mathrm{~g} / \mathrm{d}$ of plant stanols (as esters)]. There was a 2 -week washout period between the treatment periods.

Body weights did not differ after the control $(73.8 \pm 11.1 \mathrm{~kg})$, oat $\beta$-glucan $(73.9 \pm 11.0 \mathrm{~kg})$, and combination $(73.8 \pm 11.1 \mathrm{~kg})$ diet periods $(\mathrm{p}=0.997)$.

\section{Blood sampling}

The volunteers fasted overnight and were not allowed to use alcohol the day preceding or to smoke on the morning of blood sampling (63). Blood was collected in three $10 \mathrm{~mL}$ EDTA tubes (Becton Dickinson Vacutainer Systems, Breda, The Netherlands) for analysis of plasma hs-CRP concentrations and total RNA isolation. In addition, $16 \mathrm{~mL}$ of blood was sampled in 2 endotoxin-free heparinized tubes (Becton Dickinson Vacutainer Systems, Breda, The Netherlands) for PBMC and whole blood stimulation. After sampling, all 5 tubes were immediately placed on ice. EDTA Plasma was obtained directly by centrifugation of 1 EDTA tube at $2000 \times \mathrm{g}$ for $30 \mathrm{~min}$ at $4^{\circ} \mathrm{C}$. Plasma was then divided into aliquots, snapfrozen, and subsequently stored at $-80^{\circ} \mathrm{C}$ until analysis. 
CRP analysis

Hs-CRP concentrations were measured in EDTA plasma with a highly sensitive immunoturbidimetric assay (Kamiya Biomedical Company, Seattle, WA, USA) (139).

\section{PBMC and whole blood stimulation}

Heparinized blood of 30 randomly selected subjects was used to study the effects of oat $\beta$ glucan and plant stanols on leukocyte reactivity. The baseline characteristics and responses of this subgroup were comparable to that of the whole group. Leukocyte reactivity was evaluated by stimulating both PBMC as well as whole blood with LPS as described (139). Briefly, to examine ex vivo cytokine production in whole blood, 4 sterile $2 \mathrm{~mL}$ microcentrifuge tubes were each filled with $2 \mathrm{~mL}$ blood. Immediately, $20 \mu \mathrm{L}$ LPS (Escherichia coli lipopolysaccharide serotype 055:B5, Sigma Chemical Co., St. Louis, MO, USA; final concentration $10 \mathrm{ng} / \mathrm{mL}$ in endotoxin-free buffered saline) was added to 2 tubes and $20 \mu \mathrm{L}$ polymyxin B (antibiotic, Bedford Laboratories, Bedford, OH, USA; final concentration 1 $\mathrm{mg} / \mathrm{mL}$ ) to the remaining 2 tubes. Blood samples were incubated at $37^{\circ} \mathrm{C}$ for $3 \mathrm{~h}$ (determination of plasma TNF- $\alpha$ levels) or $21 \mathrm{~h}$ (determination of plasma IL-6 and IL-8 levels). After incubation, samples were centrifuged at $1000 \times \mathrm{g}$ for $30 \mathrm{~min}$ and platelet-poor plasma was stored at $-80^{\circ} \mathrm{C}$ until analysis. To examine ex vivo cytokine production by PBMC, cells were isolated from whole blood using Lymphoprep (Nycomed Pharma AS, Oslo, Norway) under sterile conditions. After isolation, PBMC of one subject were immediately plated in 4 wells of a 24-well flat-bottom culture plate $(2.5 \times 106$ cells $/ \mathrm{mL}$ per well; $150-500 \mu \mathrm{L}$ per well). For each subject, PBMC of 2 wells were mixed with $10 \mu \mathrm{L}$ LPS (final concentration $10 \mathrm{ng} / \mathrm{mL}$ ) and the remaining 2 wells with $10 \mu \mathrm{L}$ polymixin B (final concentration 1 $\mathrm{mg} / \mathrm{mL}$ ). RPMI-1640 was used as the culture medium, containing $1 \%$ penicillin/streptomycin, $1 \%$ sodium pyruvate, and $1 \%$ of a heat-inactivated human serum pool. The cells were incubated for $3 \mathrm{~h}$ (determination of plasma TNF- $\alpha$ levels) or $21 \mathrm{~h}$ (determination of plasma IL- 6 and IL-8 levels) at $37^{\circ} \mathrm{C}$. After incubation, the culture media were aspirated. The aspirated media were centrifuged at $1000 \times \mathrm{g}$ for $30 \mathrm{~min}$ to obtain cell-free media, which were stored at $-80^{\circ} \mathrm{C}$ until analysis.

\section{Cytokine analyses}

TNF- $\alpha$, IL-6, and IL-8 concentrations in platelet-poor plasma of stimulated whole blood and cell-free media of stimulated PBMC were assessed by sandwich ELISA as previously 
described (139). Briefly, plates (Greiner Bio-One, Frickenhausen, Germany) were coated with monoclonal murine antihuman TNF- $\alpha$, IL-6, and IL-8 antibodies. Recombinant human TNF- $\alpha$, IL-6, and IL-8 were used for their respective standard titration curves. Immobilized TNF- $\alpha$ was detected using a specific rabbit-anti-human TNF- $\alpha$ antibody, followed by the addition of goat-anti-rabbit peroxidase (Jackson ImmunoResearch, West Grove, PA, USA) and tetramethylbenzidine (TMB) substrate (Kirkegaard \& Perry Laboratories, Gaithersburg, MD, USA). Immobilized IL-6 or IL-8 was detected using a specific biotinylated rabbit-antihuman IL-6 or IL-8 polyclonal antibody, followed by the addition of peroxidase-conjugated streptavidin (Zymed Laboratories, San Francisco, CA, USA) and TMB substrate.

\section{Total RNA isolation}

Blood of 8 subjects, randomly selected from the subgroup of 30 subjects, was used to examine the changes in expression of 84 genes related to atherosclerosis in PBMC. This subgroup of 8 subjects was a representative sample of the original group. Blood was processed immediately after collection as gene expression might change very rapidly. PBMC were first isolated from $20 \mathrm{~mL}$ EDTA blood using Lymphoprep density-gradient centrifugation (Nycomed Pharma AS, Oslo, Norway) according to instructions of the manufacturer. After isolation, RNA was purified form the cells using $1.5 \mathrm{~mL}$ Trizol (Invitrogen Corporation, Carlsbad, CA, USA). Subsequently, samples were stored at $-80^{\circ} \mathrm{C}$ until RNA isolation.

After Trizol extraction, total RNA was further purified using RNAeasy columns (with oncolumn-DNAse treatment) according to the manufacturer's protocol (QIAGEN Benelux BV, Venlo, Netherlands). Total RNA yield and integrity was assessed using the Agilent 2100 bioanalyzer. Only samples with an RNA integrity number (RIN) $>7.0$ were used. Samples were stored at $-80^{\circ} \mathrm{C}$ until further analysis.

\section{Gene expression analyses}

The expression of 84 genes related to atherosclerosis was profiled using the $\mathrm{RT}^{2}$ Profiler ${ }^{\mathrm{TM}}$ PCR Array (Superarray, Bioscience Corporation, Frederick, USA) according to the manufacturer's protocol. Genes involved in the processes of blood coagulation and circulation were included as well as genes involved in cell-adhesion, and lipid transport and metabolism. Genes involved in the stress response, cell growth and proliferation, and apoptosis were represented as well. The expression of the 84 genes of interest plus 5 housekeeping genes (human 18S ribosomal RNA, hypoxanthine phosphoribosyltransferase 1, ribosomal protein L13a, glyceraldehydes-3-phosphate dehydrogenase, $\beta$-actin) was monitored. The 96 (SYBR 
Green) reactions were all carried out under the same cycling conditions in the same run. Data analysis was based on the $\Delta \Delta \mathrm{Ct}$ method. Briefly, the $\Delta \mathrm{Ct}$ for each pathway-focused gene in each treatment group was calculated first. The $\beta$-actin gene was used to calculate the $\Delta \mathrm{Ct}$ values. Next, the $\Delta \Delta \mathrm{Ct}$ was calculated for each gene across two groups, and used for statistical analyses. All 3 samples of one subject were analyzed in 3 runs on the same day. The correlation coefficient and efficiency of amplification were similar for all 24 standard curves of $\beta$-actin. The mean correlation coefficient for all standard curves was $0.995 \pm 0.009$. The mean slope value was $-3.543 \pm 0.263$ and the mean efficiency of detection was $90 \%$.

\section{Statistical analyses}

Normality was tested by the Shapiro-Wilk test. Hs-CRP concentrations were logtransformed to achieve normality. Log-transformed hs-CRP and cytokine concentrations were analyzed by ANOVA using the general linear model procedure. Differences in effects on the parameters of interest were examined with diet and period as fixed factors and subject number as a random factor. When a significant diet effect was found, the 3 treatments were compared pairwise using a Tukey post-hoc test for multiple comparisons of observed means. Values are presented as means \pm SD. Gene expression levels were analyzed with the non-parametric Friedman test. When a significant diet effect was found, the 3 treatments were compared pairwise using the Wilcoxon test. $\Delta \Delta \mathrm{Ct}$ are presented as medians with ranges. Differences were considered significant at $p<0.05$. Statistical analyses were performed using SPSS 11.0 (version 11.0.3) for MacIntosh OS X (version 10.3.9).

\section{RESULTS}

Hs-CRP

Mean hs-CRP concentration at the end of the control diet was $2.59 \pm 3.57$ (mean \pm SD) $\mathrm{mg} / \mathrm{L}, 2.24 \pm 4.84 \mathrm{mg} / \mathrm{L}$ at the end of the oat $\beta$-glucan diet, and $2.07 \pm 3.04 \mathrm{mg} / \mathrm{L}$ at the end of the combination diet. After log-transformation, these values were not significantly different from each other $(\mathrm{p}=0.622)$. After exclusion of 6 subjects with values $>9 \mathrm{mg} / \mathrm{L}$ (152), the end hs-CRP value of the control diet was $1.56 \pm 1.80 \mathrm{mg} / \mathrm{L}, 1.35 \pm 1.31 \mathrm{mg} / \mathrm{L}$ of the oat $\beta$-glucan diet, and $1.59 \pm 1.79 \mathrm{mg} / \mathrm{L}$ of the combination diet. Again, values did not differ significantly between the diets $(p=0.933)$. 


\section{Proinflammatory cytokine production}

Effects of the interventions on IL-6, IL-8, and TNF- $\alpha$ production in whole blood and PBMC after LPS stimulation are shown in Tables 2 and 3. Because of a small volume of supernatant, only TNF- $\alpha$ and IL- 6 concentrations were determined in the cell-free media of PBMC. In whole blood, II-6, IL-8, and TNF- $\alpha$ production did not differ significantly between the 3 dietary periods. Also in PBMC, IL- 6 and TNF- $\alpha$ production did not differ significantly between the 3 intervention groups.

TABLE 2: Effects of 4-weeks daily intake of oat $\beta$-glucan with or without plant stanols on cytokine concentrations in platelet-poor plasma of stimulated whole blood'

\begin{tabular}{lcccc}
\hline & Control & Oat $\beta$-glucan & Combination & p for diet effect ${ }^{2}$ \\
\hline TNF- $\alpha(\mathrm{ng} / \mathrm{mL})$ & $2.8 \pm 1.8$ & $2.9 \pm 2.0$ & $2.8 \pm 1.5$ & 0.905 \\
IL-6 $(\mathrm{ng} / \mathrm{mL})$ & $28.5 \pm 15.4$ & $24.8 \pm 16.9$ & $27.5 \pm 17.8$ & 0.510 \\
IL-8 $(\mathrm{ng} / \mathrm{mL})$ & $33.8 \pm 16.3$ & $34.1 \pm 16.3$ & $32.2 \pm 15.9$ & 0.533 \\
\hline
\end{tabular}

${ }^{1}$ All values are presented as means $\pm \mathrm{SD} ; \mathrm{n}=30$.

${ }^{2}$ Cytokine concentrations were analyzed by ANOVA using the general linear model procedure with subject number as a random factor and diet and period as fixed factors. Differences were considered significant at $\mathrm{p}<0.05$.

TABLE 3: Effects of 4-weeks daily intake of oat $\beta$-glucan with or without plant stanols on cytokine concentrations in cell-free media of stimulated PBMC ${ }^{1,2}$

\begin{tabular}{lcccc}
\hline & Control & Oat $\beta$-glucan & Combination & $p$ for diet effect $^{3}$ \\
\hline TNF- $\alpha(\mathrm{ng} / \mathrm{mL})$ & $0.9 \pm 0.8$ & $1.1 \pm 1.5$ & $1.0 \pm 1.1$ & 0.300 \\
IL-6 $(\mathrm{ng} / \mathrm{mL})$ & $50.9 \pm 37.2$ & $70.1 \pm 43.6$ & $71.8 \pm 62.7$ & 0.130 \\
\hline
\end{tabular}

${ }^{1} \mathrm{All}$ values are presented as means $\pm \mathrm{SD} ; \mathrm{n}=30$.

${ }^{2}$ Because of a small volume of supernatant, only TNF- $\alpha$ and IL- 6 were determined in cell-free media of PBMC. ${ }^{3}$ Cytokine concentrations were analyzed by ANOVA using the general linear model procedure with subject number as a random factor and diet and period as fixed factors. Differences were considered significant at $\mathrm{p}<0.05$.

\section{Gene expression}

Thirteen genes were not detectable (defined as requiring $>35$ cycles to obtain a measurable $\mathrm{Ct}$ ) in PBMC: APOB (apolipoprotein B), COL3A1 (collagen type-3- $\alpha 1$ ), FABP3 (fatty acid binding protein-3), FGA (fibrinogen), FN1 (fibrinonectin-1), IL3 (CSF), KDR (kinase insert domain receptor), LAMA1 (laminin- $\alpha 1)$, LPA (Lp(a)), MMP3 (matrix metalloproteinase-3), NPY (neuropeptide Y), PPARG (peroxisome proliferative activated receptor $\gamma$ ), and SPP1 (secreted phosphoprotein-1). Among the remaining 71 detectable genes, only 3 genes were differentially expressed $(p<0.05)$. These effects were only evident for the combination muesli, since supplementation with the oat $\beta$-glucan enriched muesli gave no differential 
gene expression as compared to the control muesli. The combination muesli significantly increased expression of granulocyte-macrophage colony-stimulating factor (CSF2) gene as compared both to the control $(p=0.043)$ and the oat $\beta$-glucan enriched $(p=0.043)$ mueslis (Table 4). Compared to the control muesli, expression of adipose-differentiation-related protein (ADFP; $p=0.017$ ) gene was significantly decreased by consumption of the combination muesli. This combined intake also effectively lowered expression of ADFP $(p=0.036)$ gene but only as compared to the oat $\beta$-glucan enriched muesli (Table 4). Gene expression of VE-cadherin $(\mathrm{CHH} 5)$ gene differed among the 3 treatment groups $(\mathrm{p}=0.024)$. However, between-diet comparisons were not significant.

TABLE 4: Effects of 4-weeks daily intake of $\beta$-glucan with or without plant stanols on PBMC gene expression ${ }^{1,2}$.

\begin{tabular}{ccccc}
\hline Gene & $\begin{array}{c}\Delta \Delta \mathrm{Ct} \\
(\beta \text {-Glucan - Control) }\end{array}$ & $\begin{array}{c}\Delta \Delta \mathrm{Ct} \\
\text { (Combination - Control) }\end{array}$ & $\begin{array}{c}\Delta \Delta \mathrm{Ct} \\
\text { (Combination - } \beta \text {-Glucan) }\end{array}$ & $\begin{array}{c}\mathrm{p} \text { for diet } \\
\text { effect }^{3}\end{array}$ \\
\hline ADFP & $-0.28(-0.70,0.32)$ & $0.34(-0.03,0.65)$ & $0.73(-0.35,1.10)$ & \\
& $P=0.123$ & $P=0.017^{*}$ & $P=0.036^{*}$ & $0.021^{*}$ \\
CDH5 & $0.21(0.00,0.86)$ & $0.10(0.00,0.86)$ & $0.00(-0.30,0.00)$ & $0.024^{*}$ \\
& $P=0.123$ & $P=0.068$ & $P=0.180$ & $0.010^{*}$ \\
\hline
\end{tabular}

'The expression of 84 genes related to atherosclerosis was profiled using the RT2 Profiler' $^{\text {TM }}$ PCR Array; $\mathrm{n}=8$.

${ }^{2}$ Values are $\Delta \Delta \mathrm{Ct}$ values and presented as medians with ranges.

${ }^{3}$ Gene expression levels ( $\Delta$ Ct values) were analyzed with the non-parametric Friedman test. When a significant diet effect was found, the 3 treatments were compared pairwise using the non-parametric Wilcoxon test. Differences were considered significant at $\mathrm{p}<0.05^{*}$.

\section{DISCUSSION}

Statins, commonly prescribed lipid-lowering drugs, reduce CRP levels, independently of their effects on lipid metabolism. Whether direct targeting of inflammatory components by dietary changes also lowers the risk for CVD is difficult to say, since most dietary interventions so far have aimed at lowering serum LDL cholesterol concentrations. Nevertheless, recent research has demonstrated an association between dietary fiber and levels of CRP. Viscous fiber, such as oat $\beta$-glucan, may improve CVD risk, at least partly, through improvements in serum LDL cholesterol (61). Oat $\beta$-glucan may also mediate immune and inflammatory processes $(29-33,37)$. However, data from human studies are scarce. Our results showed no effect of daily consumption of muesli enriched with $5 \mathrm{~g}$ oat $\beta$-glucan on plasma hs-CRP concentrations. Consumption of oat $\beta$-glucan had also no influence on ex vivo LPS-stimulated cytokine (IL-6, IL-8, TNF- $\alpha$ ) production in whole blood and PBMC. Nevertheless, it is not likely that the lack of effect relates to the experimental approach 
used. Previous studies have shown that effects of dietary components on cytokine production by PBMC can be detected in relatively small groups of subjects. In a study by Han et al. (140), in which only 19 subjects participated, IL-6 and TNF- $\alpha$ production by PBMC was significantly increased after consumption of a soybean oil-based stick margarine compared with soybean oil. So far, only one human study has examined the effects of oat $\beta$-glucan on inflammatory parameters. Queenan et al. (61) found, except for a significant reduction in LDL cholesterol, no significant changes in plasma CRP levels. In this study, 75 hypercholesterolemic subjects consumed either $6 \mathrm{~g} / \mathrm{d}$ concentrated oat $\beta$-glucan or $6 \mathrm{~g} / \mathrm{d}$ dextrose (control) for 6 weeks. We further found that the addition of $1.5 \mathrm{~g}$ plant stanol to the oat $\beta$ glucan enriched muesli did not change the outcomes. Like for oat $\beta$-glucan, limited information exists on the effects of plant sterols and stanols on inflammatory markers in human volunteers. In line with our results, the majority of these studies showed no significant effects on markers of inflammation, such as CRP, soluble adhesion molecules, and MCP1 (131-134). Nevertheless, Cater et al. (135) found in 13 hypercholesterolemic patients with a positive history for CVD no significant effect of plant stanols on CRP levels, but the combined administration of plant stanols with a statin significantly reduced CRP concentrations as compared to statin-treatment alone $(n=10)$. In contrast, de Jong et al. (134) found no effects of plant stanols as add-on treatment in 45 statin-users on inflammation markers. Devaraj et al. (136), however, found in 72 healthy subjects, a significant reduction in CRP levels after consumption of an orange juice enriched with plant sterols (2 $\mathrm{g} / \mathrm{d}$ ). Taken together, these studies suggest that the lack of effect on inflammation markers in the present study may not be related to the "health status" of the subjects, the dose administered, or the magnitude of the decrease in cholesterol. It should be noted that the magnitude of CRP reduction has been strongly associated with the magnitude of cholesterol lowering by various lipid-altering interventions (statins, ezetimibe, and bile acid sequestrants). It should be noted that a combination of dietary factors, such as in the Mediterranean diet (141), the Portfolio diet (142), or a prudent diet (143) effectively reduced biomarkers of inflammation. These studies were however not designed to determine the impact of individual dietary factors and the possibility of synergy between dietary factors needs to be taken into consideration. However, it cannot be excluded that a type II error (insufficient power) contributed to the lack of significant differences in the measured inflammatory markers. It is possible that a larger sample size and/or greater reduction in LDL cholesterol would have resulted in a significant CRP response.

Recent studies have shown that the RT2 PCR array is a sensitive platform to detect differential expression of "thematic" genes in different human cell types, such as alterations in genes related to drug metabolism in primary hepatocytes (144) and genes related to Th2 immune response in lymphocytes isolated from allergic subjects (145). More detailed characterization of diet-induced differential expression of genes involved in atherosclerosis may enhance the understanding of the effects of oat $\beta$-glucan and plant stanols on CVD 
risk. PBMC may be surrogate target cells to monitor effects in target tissues (146). Daily consumption of muesli enriched with $5 \mathrm{~g}$ oat $\beta$-glucan did, however, not change gene expression of an atherosclerotic risk panel of 84 genes as measured in PBMC. Addition of $1.5 \mathrm{~g}$ plant stanols increased expression of CSF2 gene (pro-inflammatory effect), and decreased expression of ADFP gene (anti-inflammatory effect) in PBMC as compared to both the control and the oat $\beta$-glucan enriched mueslis. The CSF2 gene encodes granulocyte-macrophage colony-stimulating factor (GMCSF). CSF2 plays an important role in smooth muscle cell-dependent monocyte activation (147). The ADFP gene encodes adipose differentiation-related protein (ADRP). ADRP is a membrane-associated protein whose mRNA levels are induced rapidly and maximally after triggering adipocyte differentiation. ADFP is highly expressed in advanced lipid-enriched human atherosclerotic plaques (148). However, giving functional significance to changes in gene expression level alone requires caution, as it provides no information on post-translational modifications or the rate of protein degradation. In addition, mRNA levels do not always correlate with protein synthesis. It should be noted, however, that the alterations found in gene expression after addition of plant stanols may have occurred by chance. If 84 genes are tested with a probability threshold of $0.05,4$ genes $(84 \times 0.05)$ are expected to be significant by chance alone. Nevertheless, it is known that gene expression in PBMC can change after consumption of plant stanols (19). In that study, a daily consumption of $4 \mathrm{~g}$ plant stanols increased LDL receptor mRNA concentrations in mononuclear blood cells by $43 \%$ as measured by competitive RT-PCR. A reason for our lack of effect on the LDL receptor gene could be the difference in the dose administered and the concomitant reduction in serum LDL cholesterol concentrations.

In conclusion, the current level of supplementation of oat $\beta$-glucan and plant stanols as their fatty acid esters in the present study effectively lowered serum LDL cholesterol levels in slightly hypercholesterolemic subjects, but seemed to have no effects on inflammatory markers related to atherosclerosis and CVD.

\section{ACKNOWLEDGEMENTS}

We thank the study participants for their cooperation and enthusiasm. We also thank the technical and dietary staff from our Department for their support. 


\section{CHAPTER 5}

\section{Plant stanol consumption decreases serum triacylglycerols in subjects with overt hypertriglyceridemia}

Elke Theuwissen, Jogchum Plat, Carla J.H. van der Kallen, Marleen M.J. van Greevenbroek, Ronald P. Mensink

Submitted 


\begin{abstract}
Objective Evidence is accumulating that high serum concentrations of triacylglycerols (TAG) are, like LDL cholesterol, causally related to CVD. A recent meta-analysis has indicated that plant stanols not only lower serum LDL cholesterol, but also serum TAG concentrations, especially in subjects with high baseline TAG concentrations. We therefore evaluated the effects of plant stanols on lipid and lipoprotein metabolism in a population with elevated fasting serum TAG concentrations.
\end{abstract}

Study design In a randomized, placebo-controlled, parallel study, 28 subjects with elevated TAG concentrations $(>1.7 \mathrm{mmol} / \mathrm{L})$ were studied. After a 1-week run-in during which control margarine $(60 \%$ absorbable fats) was used, subjects consumed for 3 weeks either control or plant stanol-enriched margarine $[2.4 \pm 0.2 \mathrm{~g} / \mathrm{d}$ (mean $\pm \mathrm{SD}$ ) plant stanols].

Results Plant stanol consumption significantly decreased serum total $(6.7 \%, p=0.015)$ and LDL cholesterol $(9.5 \%, \mathrm{p}=0.041)$. A significant interaction between baseline serum TAG concentrations and plant stanol intake was found: intake of plant stanols lowered TAG concentrations, particularly in subjects with high baseline TAG concentrations $(>2.3$ $\mathrm{mmol} / \mathrm{L} ; \mathrm{p}=0.009$ for the interaction between baseline TAG concentrations and plant stanol intake). Additionally, a significant interaction between baseline total number of LDL particles (LDL-P) and plant stanol intake was found $(p=0.020)$. Plant stanol consumption lowered LDL-P, primarily in subjects with elevated baseline values, mainly due to a nonsignificant decrease in the number of atherogenic small LDL subclass particles (small LDLP).

Conclusion Plant stanol supplementation not only lowered serum LDL cholesterol, but also serum TAG concentrations, especially in subjects with overt hypertriglyceridemia. 


\section{INTRODUCTION}

The link between high serum low-density lipoprotein (LDL) cholesterol concentrations and cardiovascular disease (CVD) has been clearly established (39). However, evidence is accumulating that high serum concentrations of triacylglycerols (TAG) - also known as hypertriglyceridemia - and low serum concentrations of high-density lipoprotein (HDL) cholesterol are also causally related to CVD (39). High TAG and low HDL concentrations often occur together, frequently with normal concentrations of LDL cholesterol, increased concentrations of small dense LDL (smallèè LDL-P) and apolipoprotein B (apoB), and insulin resistance. This lipid abnormality is a fundamental characteristic of patients with the metabolic syndrome (MS) (39).

Interestingly, a recent meta-analysis has indicated that consumption of plant stanols not only lowers serum LDL cholesterol, but also serum TAG concentrations, in particular in subjects with high baseline TAG concentrations (40). The reason that these effects have not been observed in individual studies may have been due to a lack of statistical power, as effects were only marginal in subjects with normal serum TAG concentrations. We therefore decided to design for the first time a study to specifically evaluate the effects of plant stanols on the plasma lipoprotein profile in a population with elevated fasting serum TAG concentrations.

\section{MATERIALS AND METHODS}

\section{Study population}

Subjects, aged between 18 and $70 \mathrm{y}$, were recruited among an already existing cohort of patients diagnosed with familial combined hyperlipidemia (FCHL) at the Academic Hospital Maastricht (AZM). Patients were characterized by a specific phenotype, i.e. serum total cholesterol concentrations $>6.5 \mathrm{mmol} / \mathrm{L}$ and/or elevated serum TAG concentrations $(>2.3 \mathrm{mmol} / \mathrm{L})$ at different visits before taking medication. Also subjects, who had serum TAG concentrations between $1.7 \mathrm{mmol} / \mathrm{L}$ and $4.0 \mathrm{mmol} / \mathrm{L}$, as indicated in earlier studies at our Department, were approached. A fasting serum TAG concentration $>1.7 \mathrm{mmol} / \mathrm{L}$ was chosen as lower boundary as higher concentrations are considered to be elevated (149). Subjects with concentrations $>4.0 \mathrm{mmol} / \mathrm{L}$ need to be treated with medication and were therefore excluded (150). Further inclusion criteria were: no history of CVD like congestive heart failure or recent ( $<6$ months) event (acute myocardial infarction, stroke), type I and II diabetes mellitus, epilepsy, asthma, COPD, inflammatory bowel diseases, cancer, or rheumatoid arthritis; no use of diuretics; no abuse of drugs and/or alcohol; willing to 
abstain from alcohol 3 days before blood sampling; no pregnant or breast-feeding women; no use of an investigational product 30 days before the study; no participation in another biomedical study 30 days before the study. Blood donation or participation in another biomedical trial was not allowed 30 days before and during the study. The Medical Ethical Committee of the University of Maastricht had approved the study. Participants were given a detailed description of the experimental protocol and purpose of the study before they gave their written informed consent.

Twenty-nine volunteers were selected for the study. One subject withdrew in the $3^{\text {rd }}$ week of the study because of difficulty in performing venipuncture. All other 28 volunteers, 16 men and 12 women, completed the study (Table $\mathbf{1}$ ).

TABLE 1: Baseline characteristics'1.

\begin{tabular}{lccc}
\hline & All & Control diet & Stanol diet \\
\hline Number $(\mathrm{M} / \mathrm{F})$ & $28(16 / 12)$ & $14(8 / 6)$ & $14(8 / 6)$ \\
Age $(\mathrm{y})$ & $54 \pm 8$ & $55 \pm 9$ & $53 \pm 8$ \\
$\mathrm{BMI}\left(\mathrm{kg} / \mathrm{m}^{2}\right)$ & $28 \pm 3$ & $28 \pm 3$ & $29 \pm 4$ \\
Systolic blood pressure $(\mathrm{mmHg})$ & $131 \pm 13$ & $132 \pm 12$ & $131 \pm 14$ \\
Diastolic blood pressure $(\mathrm{mmHg})$ & $87 \pm 8$ & $89 \pm 9$ & $86 \pm 6$ \\
Total cholesterol (mmol/L) & $7.17 \pm 0.96$ & $7.02 \pm 1.14$ & $7.32 \pm 0.76$ \\
LDL cholesterol $(\mathrm{mmol} / \mathrm{L})$ & $4.75 \pm 1.01$ & $4.67 \pm 1.00$ & $5.03 \pm 0.77$ \\
HDL cholesterol $(\mathrm{mmol} / \mathrm{L})$ & $1.27 \pm 0.33$ & $1.32 \pm 0.43$ & $1.21 \pm 0.17$ \\
TAG $(\mathrm{mmol} / \mathrm{L})$ & $2.62 \pm 0.68$ & $2.61 \pm 0.59$ & $2.63 \pm 0.77$ \\
Smokers & 4 & 0 & 4 \\
Lipid-lowering medication $^{2}$ & 5 & 2 & 3 \\
\hline
\end{tabular}

Values are means $\pm \mathrm{SD} ; \mathrm{n}=28$.

${ }^{2}$ Subjects had to stop the intake of their lipid-lowering medication 2 weeks before the actual start of the study and during the 4 weeks of the study.

\section{Study design}

The study had a randomized, double-blind, placebo-controlled, parallel design. Two weeks before the start of the actual study and during the 4 weeks of the study, subjects had to stop (in consultation with their general practitioner) the intake of their regular cholesterollowering medication (when appropriate). During the first 2 weeks of the study (before the run-in period), subjects were given a control margarine containing $60 \%$ absorbable fats. After these 2 weeks, further participation (and thus continuation of the medication-free period) did only take place if serum total cholesterol concentrations were $<8.0 \mathrm{mmol} / \mathrm{L}$ and TAG concentrations $<4.0 \mathrm{mmol} / \mathrm{L}$ (150). These subjects continued to consume daily $20 \mathrm{~g}$ of the control margarine for one more week. At the end of this run-in period, subjects were randomly divided over 2 groups, stratified by gender. The following 3 weeks (experimental period), one group continued to consume the control margarine, while the other group 
consumed daily $20 \mathrm{~g}$ of the experimental margarine, which had a similar fat content and fatty acid composition as the control margarine, but provided $2.5 \mathrm{~g}$ of plant stanols. Plant stanols were made by saturation of plant sterols from tall oil, giving sitostanol $(77.7 \%)$, campestanol $(16.7 \%)$, sitosterol $(2.2 \%)$, campesterol $(1.8 \%)$, brassicasterol $(0.1 \%)$, stigmasterol $(0.1 \%)$, and other phytosterols $(1.4 \%)$ (Raisio, Finland). The free plant stanols were esterified with sunflower-oil fatty acids. The margarine was packed in tubs of $140 \mathrm{~g}$, equivalent to margarine for 7 days. All products were color-coded to blind the subjects and the investigators. All margarine leftovers had to be returned and were weighed back to estimate average daily margarine intake.

Participants recorded in diaries any signs of illness, medication used, menstrual phase, alcohol consumption, any deviations of the study protocol, and any experienced complaints. Subjects also recorded their food intake for the previous 3 weeks at days 21 and 42 by completing food-frequency questionnaires (FFQ) to estimate their energy and nutrient intakes (Table 2). Body weight without shoes and heavy clothes was recorded at each visit.

\section{Blood sampling}

During the study, five fasting blood samples (days 14, 21, 32, 37, and 42) from a forearm vein using vacutainers under minimal stasis with the subject in a supine position were drawn. On the morning of sampling, subjects were not allowed to smoke, to eat, or to drink (except water). Volunteers were fasting since 22:00 the day preceding blood sampling. In addition, subjects were not allowed to drink alcohol 3 days before sampling. Blood was collected in a $10 \mathrm{~mL}$ serum tube (Becton Dickinson Vacutainer Systems, Breda, The Netherlands) for analysis of lipids, apolipoproteins, phytosterols, insulin, highsensitivity C-reactive protein (hs-CRP), and indices of liver function. Serum tubes were kept at room temperature after blood sampling. At least $1 \mathrm{~h}$ after venipuncture, serum was obtained by centrifuging at $2000 \times \mathrm{g}$ for $30 \mathrm{~min}$ at $4{ }^{\circ} \mathrm{C}$. At the same time, $4 \mathrm{~mL}$ of blood was also collected in a NaFl tube (Becton Dickinson Vacutainer Systems, Breda, The Netherlands) for analysis of glucose. Plasma was obtained immediately by centrifugation at $2000 \times \mathrm{g}$ for $30 \mathrm{~min}$ at $4^{\circ} \mathrm{C}$. At days 21 and 42, blood was also collected in a $10 \mathrm{~mL}$ EDTA tube (Becton Dickinson Vacutainer Systems, Breda, The Netherlands) for analysis of the plasma lipoprofile (number of lipoprotein particles). EDTA plasma was obtained directly by centrifugation of the EDTA tube at $2000 \times \mathrm{g}$ for $30 \mathrm{~min}$ at $4^{\circ} \mathrm{C}$. Serum and plasma were divided into aliquots, snap-frozen, and subsequently stored at $-80^{\circ} \mathrm{C}$ until analysis. All samples from one subject were analyzed in the same analytical run. 
Side effects

Serum samples of days 21 and 42 were analyzed to determine hs-CRP (Kamiya Biomedical Company, Seattle, WA, USA) and parameters of liver function (alanine transaminase, aspartate aminotransferase, $\gamma$-glutamyl transpeptidase, alkaline phosphatase, and total bilirubin) by routine procedures.

\section{Lipid and lipoprotein metabolism}

Concentrations of total cholesterol (ABX Diagnostics, Montpellier, France), HDL cholesterol (precipitation method; Roche Diagnostics Corporation, Indianapolis, IN, USA), and TAG corrected for free glycerol (Sigma-Aldrich Chemie, Steinheim, Germany) were analyzed in all serum samples. Apolipoprotein concentrations (apoA-I, apoB-100) were analyzed in serum samples from days 14, 21, 37, and 42 (ABX Diagnostics, Montpellier, France). Serum LDL cholesterol concentrations were calculated using the Friedewaldequation (112). The plasma lipoprofile (number of lipoprotein particles) was analyzed by NMR (NMR LipoProfile test, Liposcience Inc., Raleigh, NC, USA).

\section{Glucose metabolism}

Plasma samples of days 14, 21, 37, and 42 were analyzed to determine glucose concentrations (Roche Diagnostic Systems, Hoffmann-La Roche Ltd., Basel, Switzerland). Insulin was measured using an ELISA method (DRG Instruments GmbH, Germany). The HOMA index, a measure of insulin sensitivity, was calculated as described (151).

\section{Serum plant sterols and stanols}

Plant sterols and stanols (campestanol, sitostanol, campesterol, sitosterol, lathosterol) were determined in serum samples of days 21 and 42 as described (111).

\section{Statistical analyses}

Before the statistical analyses, concentrations of serum lipid and lipoproteins, glucose and insulin from days 14 and 21, and days 37 and 42 were averaged for each subject. Normality was tested by the Shapiro-Wilk test. Hs-CRP concentrations and indices of liver function 
were not normally distributed and analyzed accordingly by the non-parametric MannWhitney test. Differences in baseline characteristics between the control and intervention groups, and differences in changes in dietary intakes were analyzed by the independent Student's T test. Metabolic parameters were analyzed by ANCOVA. To answer the question whether baseline subject characteristics influenced response-to-treatment, the baseline value of that variable was included as a covariate into the model together with an interaction term (baseline value $x$ treatment). The interaction term was only included in the final statistical model if statistically significant. Values are presented as means \pm SD. Pearson correlation coefficients were determined for the relationship between the plant stanolinduced changes in TAG and the changes in the different lipoprotein particles. Individuals were also stratified for low and high serum baseline TAG concentrations (149). Due to nonnormality of the data, these values are presented as medians with ranges. Differences were considered statistically significant at $\mathrm{p}<0.05$. Statistical analyses were performed using SPSS 11.0 (version 11.0.3) for Maclntosh OS X (version 10.3.9).

Inspection of the diaries revealed that 5 subjects had consumed alcohol during the 3-day period before blood sampling. For this reason, their corresponding TAG values for that particular day were omitted from analyses. In addition, one subject had to be excluded from the statistical analyses, because of alcohol consumption at days 14, 37, and 42 . Another subject was excluded, because he continued his use of lipid-lowering medication during part of the study. Therefore, data of 26 subjects were used in the statistical analyses.

\section{RESULTS}

\section{Side effects}

Throughout the study, all variables related to liver function remained within the normal range for all subjects and no treatment effects were present (data not shown). Also concentrations of hs-CRP did not change during the study. Five subjects had, unrelated to the dietary treatments, on one (or more) occasion(s) hs-CRP concentrations $>9 \mathrm{mg} / \mathrm{L}$ (152). When these subjects were excluded from the analyses, conclusions did not change (data not shown).

\section{Dietary intake and body weight}

Average daily consumption of margarine on the control and plant stanol diets was $19.3 \pm$ $1.9 \mathrm{~g}$ and $19.3 \pm 1.8 \mathrm{~g}$, respectively. Average daily plant stanol intake was therefore $2.41 \pm$ 
0.23 g. Changes in daily energy intake and nutrient composition of the diets did not differ among the treatment groups (Table 2). Also changes in body weight were similar for the control and plant stanol groups $(p=0.579)$, and were $-0.10 \pm 0.92 \mathrm{~kg}$ and $0.11 \pm 0.74 \mathrm{~kg}$, respectively.

TABLE 2: Nutrient composition of the two diets according to FFQ1.

\begin{tabular}{|c|c|c|c|}
\hline & Run-in period & Test period & $p$ value $^{2}$ \\
\hline \multicolumn{4}{|l|}{$\overline{\text { Energy (MJ) }}$} \\
\hline Control diet & $10.0 \pm 2.0$ & $10.3 \pm 2.9$ & \multirow{2}{*}{0.167} \\
\hline Stanol diet & $8.9 \pm 2.3$ & $8.6 \pm 2.1$ & \\
\hline \multicolumn{4}{|l|}{ Protein (en\%) } \\
\hline Control diet & $17.5 \pm 2.5$ & $17.1 \pm 2.3$ & \multirow{2}{*}{0.697} \\
\hline Stanol diet & $17.3 \pm 2.3$ & $17.2 \pm 2.2$ & \\
\hline \multicolumn{4}{|l|}{ Total fat (en\%) } \\
\hline Control diet & $35.7 \pm 4.6$ & $35.8 \pm 1.3$ & \multirow{2}{*}{0.583} \\
\hline Stanol diet & $35.5 \pm 4.1$ & $36.0 \pm 3.6$ & \\
\hline \multicolumn{4}{|l|}{ SFA (en\%) } \\
\hline Control diet & $12.5 \pm 2.5$ & $12.5 \pm 2.6$ & \multirow{2}{*}{0.656} \\
\hline Stanol diet & $11.9 \pm 1.5$ & $12.2 \pm 1.1$ & \\
\hline \multicolumn{4}{|l|}{ MUFA (en\%) } \\
\hline Control diet & $11.7 \pm 1.6$ & $11.7 \pm 1.6$ & \multirow{2}{*}{0.865} \\
\hline Stanol diet & $11.6 \pm 2.0$ & $11.6 \pm 2.0$ & \\
\hline \multicolumn{4}{|l|}{ PUFA (en\%) } \\
\hline Control diet & $8.0 \pm 1.5$ & $8.0 \pm 1.8$ & \multirow{2}{*}{0.560} \\
\hline Stanol diet & $9.1 \pm 1.8$ & $9.3 \pm 1.7$ & \\
\hline \multicolumn{4}{|c|}{ Carbohydrates (en\%) } \\
\hline Control diet & $44.7 \pm 4.0$ & $44.5 \pm 4.5$ & \multirow{2}{*}{0.975} \\
\hline Stanol diet & $45.0 \pm 5.8$ & $44.9 \pm 6.0$ & \\
\hline \multicolumn{4}{|l|}{ Fiber (g/MJ) } \\
\hline Control diet & $3.0 \pm 0.8$ & $2.9 \pm 0.9$ & \multirow{2}{*}{0.603} \\
\hline Stanol diet & $3.0 \pm 0.5$ & $2.9 \pm 0.6$ & \\
\hline \multicolumn{4}{|l|}{ Alcohol (en\%) } \\
\hline Control diet & $1.7 \pm 2.3$ & $2.2 \pm 2.4$ & \multirow{2}{*}{0.373} \\
\hline Stanol diet & $1.9 \pm 3.0$ & $1.8 \pm 3.5$ & \\
\hline \multicolumn{4}{|c|}{ Cholesterol (mg/MJ) } \\
\hline Control diet & $22.2 \pm 5.2$ & $21.0 \pm 5.4$ & \multirow{2}{*}{0.446} \\
\hline Stanol diet & $22.0 \pm 4.3$ & $21.7 \pm 5.4$ & \\
\hline
\end{tabular}

${ }^{1}$ Values are means $\pm \mathrm{SD} ; \mathrm{n}=26$.

${ }^{2}$ Changes were analyzed by the independent Student's T test. Differences were considered statistically significant at $\mathrm{p}<0.05$. 


\section{Lipid and lipoprotein metabolism}

Compared to the control diet, consumption of plant stanols significantly decreased serum total and LDL cholesterol concentrations by $6.7 \%(p=0.015)$ and $9.5 \%(p=0.041)$, respectively (Table 3). In addition, serum apoB-100 concentrations were significantly lowered by $7.1 \%(p=0.007)$. Changes in serum HDL cholesterol and apoA-1 concentrations did not differ among the diet groups. A significant interaction between baseline TAG value and plant stanol intake was found $(\mathrm{p}=0.009)$.

TABLE 3: Effects of plant stanol consumption on serum lipids and lipoproteins'.

\begin{tabular}{|c|c|c|c|c|c|}
\hline & \multirow[t]{2}{*}{ Run-in period } & \multirow[t]{2}{*}{ Test period } & \multicolumn{3}{|c|}{$p$ value ${ }^{2}$} \\
\hline & & & Baseline & Diet & Baseline $x$ diet \\
\hline \multicolumn{6}{|c|}{ Total cholesterol } \\
\hline Control diet & $7.14 \pm 0.89$ & $7.13 \pm 1.03$ & \multirow{2}{*}{0.866} & \multirow{2}{*}{0.015} & \multirow{2}{*}{-} \\
\hline Stanol diet & $7.35 \pm 0.67$ & $6.85 \pm 0.73$ & & & \\
\hline \multicolumn{6}{|l|}{ LDL cholesterol } \\
\hline Control diet & $4.63 \pm 0.89$ & $4.62 \pm 0.97$ & \multirow{2}{*}{0.265} & \multirow{2}{*}{0.041} & \multirow{2}{*}{-} \\
\hline Stanol diet & $4.96 \pm 0.70$ & $4.48 \pm 0.66$ & & & \\
\hline \multicolumn{6}{|c|}{ HDL cholesterol } \\
\hline Control diet & $1.30 \pm 0.41$ & $1.19 \pm 0.40$ & \multirow{2}{*}{0.271} & \multirow{2}{*}{0.586} & \multirow{2}{*}{-} \\
\hline Stanol diet & $1.20 \pm 0.18$ & $1.18 \pm 0.17$ & & & \\
\hline \multicolumn{6}{|l|}{ Triacylglycerols } \\
\hline Control diet & $2.63 \pm 0.58$ & $2.71 \pm 0.71$ & \multirow{2}{*}{ - } & \multirow{2}{*}{ - } & \multirow{2}{*}{0.009} \\
\hline Stanol diet & $2.52 \pm 0.66$ & $2.50 \pm 0.47$ & & & \\
\hline \multicolumn{6}{|l|}{ ApoB-100 } \\
\hline Control diet & $1.28 \pm 0.23$ & $1.30 \pm 0.22$ & \multirow{2}{*}{0.322} & \multirow{2}{*}{0.007} & \multirow{2}{*}{ - } \\
\hline Stanol diet & $1.36 \pm 0.16$ & $1.28 \pm 0.16$ & & & \\
\hline \multicolumn{6}{|l|}{ ApoA-1 } \\
\hline Control diet & $1.46 \pm 0.34$ & $1.46 \pm 0.35$ & \multirow{2}{*}{0.746} & \multirow{2}{*}{0.666} & \multirow{2}{*}{-} \\
\hline Stanol diet & $1.42 \pm 0.19$ & $1.41 \pm 0.19$ & & & \\
\hline
\end{tabular}

${ }^{1}$ Values are means $\pm S D(n=26)$ and are expressed in mmol/L, except for apoB-100 and apoA-1 which are expressed in $\mathrm{mg} / \mathrm{L}$. Days 14 and 21 were averaged for the end of the run-in period. Days 37 and 42 were averaged for the end of the experimental period.

${ }^{2}$ Data were analyzed by ANCOVA. To answer the question whether baseline subject characteristics influenced response-to-treatment, the baseline value of the variable of interest was included (as a covariate) into the model together with an interaction term (baseline value $\mathrm{x}$ diet). The interaction term was only included in the statistical model if statistically significant. For the control group, diet was coded with a " 0 " and for the intervention group with a "1". Differences were considered statistically significant at $\mathrm{p}<0.05$.

To further explore this significant interaction, we stratified the individuals for low and high baseline TAG concentrations (149). Plant stanol consumption lowered TAG concentrations $(11 \%, p=0.051)$ in subjects with high baseline TAG concentrations $(>2.3 \mathrm{mmol} / \mathrm{L}$; Figure 
1). For subjects with lower baseline serum TAG concentrations $(<2.3 \mathrm{mmol} / \mathrm{L})$, plant stanol consumption did not affect serum TAG concentrations $(p=0.909)$.

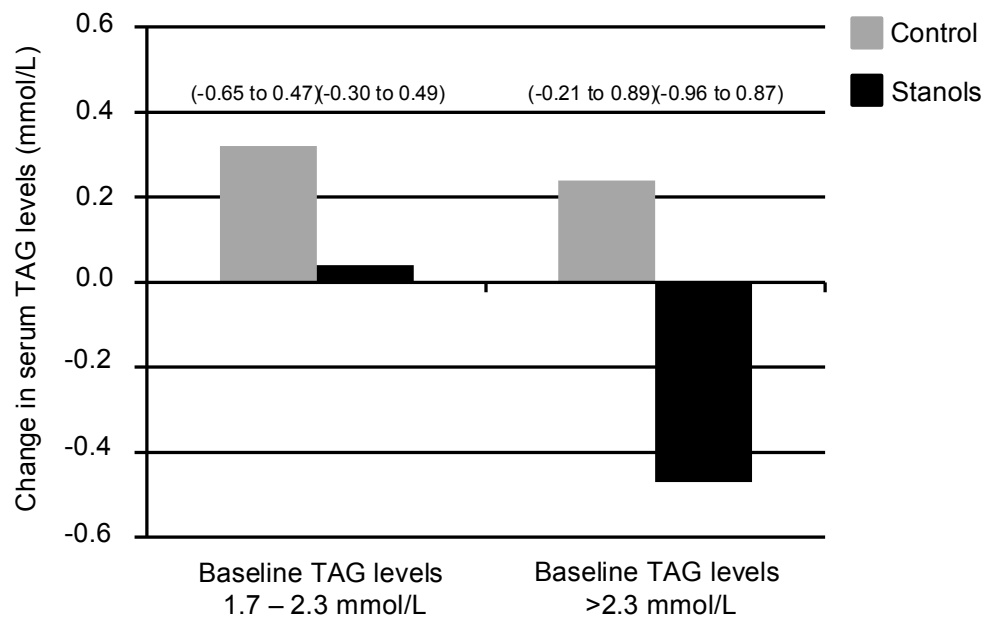

FIGURE 1. Interaction between baseline serum TAG values and response-to-treatment.

The median change (with ranges) in serum TAG levels $(\mathrm{mmol} / \mathrm{L}$ ) is plotted for 2 groups of subjects; subjects with baseline TAG values between $1.7 \mathrm{mmol} / \mathrm{L}$ and $2.3 \mathrm{mmol} / \mathrm{L}(\mathrm{n}=10)$ and subjects with baseline values $>2.3 \mathrm{mmol} / \mathrm{L}(\mathrm{n}$ = 16). Plant stanols lowered serum TAG levels, particularly in subjects with high baseline TAG levels $(>2.3 \mathrm{mmol} / \mathrm{L})$.

Further, a significant interaction between baseline total number of LDL particles (LDL-P) and plant stanol intake was found ( $\mathrm{p}=0.020$; Table 4). Supplementation of plant stanols lowered LDL-P, primarily in subjects with elevated baseline values. This decrease was reflected by a reduction of similar magnitude in the number of small LDL-subclass particles (small LDL-P), but this decline did not reach statistical significance $(\mathrm{p}=0.150)$. 
TABLE 4: Effects of plant stanol consumption on the plasma NMR lipoprofile'.

\begin{tabular}{|c|c|c|c|c|c|}
\hline & \multirow[t]{2}{*}{ Run-in period } & \multirow[t]{2}{*}{ Test period } & \multicolumn{3}{|c|}{ p value ${ }^{2}$} \\
\hline & & & Baseline & Diet & Baseline $x$ diet \\
\hline \multicolumn{6}{|l|}{$\begin{array}{l}\text { Total VLDL } \\
\text {. }\end{array}$} \\
\hline Control diet & $107 \pm 29$ & $106 \pm 28$ & 0.040 & 0.643 & - \\
\hline Stanol diet & $117 \pm 29$ & $116 \pm 29$ & & & \\
\hline \multicolumn{6}{|l|}{ Large VLDL } \\
\hline Control diet & $13 \pm 6$ & $12 \pm 5$ & 0.028 & 0.897 & - \\
\hline Stanol diet & $11 \pm 7$ & $13 \pm 10$ & & & \\
\hline \multicolumn{6}{|l|}{ Medium VLDL } \\
\hline Control diet & $46 \pm 20$ & $53 \pm 20$ & 0.007 & 0.322 & - \\
\hline Stanol diet & $54 \pm 22$ & $51 \pm 16$ & & & \\
\hline \multicolumn{6}{|l|}{ Small VLDL } \\
\hline Control diet & $48 \pm 18$ & $41 \pm 17$ & 0.052 & 0.148 & - \\
\hline Stanol diet & $52 \pm 19$ & $52 \pm 26$ & & & \\
\hline \multicolumn{6}{|l|}{ Total LDL } \\
\hline Control diet & $1784 \pm 413$ & $1791 \pm 519$ & - & - & 0.020 \\
\hline Stanol diet & $1901 \pm 337$ & $1688 \pm 306$ & & & \\
\hline \multicolumn{6}{|l|}{ IDL } \\
\hline Control diet & $78 \pm 57$ & $99 \pm 55$ & 0.015 & 0.368 & - \\
\hline Stanol diet & $105 \pm 44$ & $82 \pm 58$ & & & \\
\hline \multicolumn{6}{|l|}{ Large LDL } \\
\hline Control diet & $240 \pm 197$ & $224 \pm 189$ & 0.009 & 0.600 & - \\
\hline Stanol diet & $175 \pm 158$ & $168 \pm 137$ & & & \\
\hline \multicolumn{6}{|l|}{ Small LDL } \\
\hline Control diet & $1466 \pm 471$ & $1468 \pm 531$ & 0.277 & 0.150 & - \\
\hline Stanol diet & $1621 \pm 364$ & $1438 \pm 320$ & & & \\
\hline \multicolumn{6}{|l|}{ Total HDL } \\
\hline Control diet & $30 \pm 7$ & $31 \pm 8$ & 0.873 & 0.268 & - \\
\hline Stanol diet & $31 \pm 4$ & $31 \pm 3$ & & & \\
\hline \multicolumn{6}{|l|}{ Large HDL } \\
\hline Control diet & $4.7 \pm 3.6$ & $4.7 \pm 3.7$ & 0.341 & 0.728 & - \\
\hline Stanol diet & $3.6 \pm 2.1$ & $3.8 \pm 2.0$ & & & \\
\hline \multicolumn{6}{|l|}{ Medium HDL } \\
\hline Control diet & $5.6 \pm 4.8$ & $5.3 \pm 2.9$ & 0.004 & 0.992 & - \\
\hline Stanol diet & $4.2 \pm 3.3$ & $5.0 \pm 4.7$ & & & \\
\hline \multicolumn{6}{|l|}{ Small HDL } \\
\hline Control diet & $20 \pm 6$ & $21 \pm 6$ & 0.052 & 0.748 & - \\
\hline Stanol diet & $24 \pm 3$ & $23 \pm 4$ & & & \\
\hline
\end{tabular}

${ }^{1}$ Values are means $\pm \mathrm{SD}(\mathrm{n}=26)$ and are expressed in $\mathrm{nmol} / \mathrm{L}$, except for HDL particles which are expressed in $\mu \mathrm{mol} / \mathrm{L}$.

${ }^{2}$ Data were analyzed by ANCOVA. To answer the question whether baseline subject characteristics influenced response-to-treatment, the baseline value of the variable of interest was included (as a covariate) into the model together with an interaction term (baseline value $\mathrm{x}$ diet). The interaction term was only included in the statistical model if statistically significant. For the control group, diet was coded with a " 0 " and for the intervention group with $\mathrm{a}$ "1". Differences were considered statistically significant at $\mathrm{p}<0.05$. 


\section{Serum sterols and stanols}

Cholesterol-standardized serum sterol and stanol concentrations are depicted in Table 5. A significant interaction between baseline concentrations of cholesterol-standardized sitostanol and response-to-treatment was found $(\mathrm{p}=0.011)$. Consumption of plant stanols increased cholesterol-standardized sitostanol concentrations, in particular in subjects with elevated baseline values. Additionally, eating the plant stanol-enriched margarine significantly increased cholesterol-standardized serum campestanol concentrations $(p<0.001)$. Plant stanol concentrations increased in every subject receiving the plant stanol-enriched margarines, which demonstrates good compliance with the study protocol.

TABLE 5: Serum sterols and stanols (corrected for serum cholesterol as measured by GC) ${ }^{1}$.

\begin{tabular}{|c|c|c|c|c|c|}
\hline & \multirow[t]{2}{*}{ Run-in period } & \multirow[t]{2}{*}{ Test period } & \multicolumn{3}{|c|}{$\mathrm{p}$ value ${ }^{2}$} \\
\hline & & & Baseline & Diet & Baseline $x$ diet \\
\hline \multicolumn{6}{|l|}{ Sitostanol } \\
\hline Control diet & $2.58 \pm 0.70$ & $2.41 \pm 0.62$ & - & - & 0.011 \\
\hline Stanol diet & $2.51 \pm 0.69$ & $22.97 \pm 7.60$ & & & \\
\hline \multicolumn{6}{|l|}{ Campestanol } \\
\hline Control diet & $1.12 \pm 0.49$ & $1.12 \pm 0.42$ & 0.270 & $<0.001$ & - \\
\hline Stanol diet & $1.20 \pm 0.56$ & $10.06 \pm 3.58$ & & & \\
\hline \multicolumn{6}{|l|}{ Sitosterol } \\
\hline Control diet & $129 \pm 31$ & $123 \pm 27$ & 0.007 & 0.008 & - \\
\hline Stanol diet & $141 \pm 47$ & $114 \pm 39$ & & & \\
\hline \multicolumn{6}{|l|}{ Campesterol } \\
\hline Control diet & $159 \pm 41$ & $154 \pm 42$ & 0.021 & 0.020 & - \\
\hline Stanol diet & $183 \pm 73$ & $152 \pm 61$ & & & \\
\hline \multicolumn{6}{|l|}{ Lathosterol } \\
\hline Control diet & $188 \pm 64$ & $195 \pm 46$ & - & - & 0.043 \\
\hline Stanol diet & $184 \pm 54$ & $210 \pm 65$ & & & \\
\hline
\end{tabular}

${ }^{1}$ Values are means \pm SD $(n=26)$ and are expressed in $(\mu \mathrm{mol} / \mathrm{mmol}$ cholesterol $) \times 100$.

${ }^{2}$ Data were analyzed by ANCOVA. To answer the question whether baseline subject characteristics influenced response-to-treatment, the baseline value of the variable of interest was included (as a covariate) into the model together with an interaction term (baseline value $\mathrm{x}$ diet). The interaction term was only included in the statistical model if statistically significant. For the control group, diet was coded with a " 0 " and for the intervention group with a "1". Differences were considered statistically significant at $\mathrm{p}<0.05$.

Compared to the control diet, cholesterol-standardized serum sitosterol $(p=0.008)$ and campesterol $(p=0.020)$ concentrations significantly decreased after plant stanol consumption, indicating decreased intestinal cholesterol absorption. A significant interaction between baseline cholesterol-standardized lathosterol concentrations and plant stanol intake was found $(p=0.043)$. Daily intake of plant stanols increased this marker for endogenous cholesterol synthesis, especially in subjects with elevated baseline values. 


\section{Glucose metabolism}

Changes in plasma glucose and insulin concentrations, and HOMA index did not differ among the diet groups (data not shown).

\section{Correlations}

Significant correlations were found between plant stanol-induced changes in serum concentrations of TAG and the number of large VLDL-subclass particles (large VLDL-P; $r=0.713, p<0.001)$, large LDL-P $(r=-0.575, p=0.002)$, and small LDL-P $(r=0.438, p=0.025)$. Further, plant stanol-induced changes in serum TAG levels significantly correlated with the number of medium HDL-subclass particles (medium HDL-P; $r=0.394, p=0.047$ ). Regarding the remaining lipoprotein particles, no significant correlations were found with the plant stanol-induced changes in serum TAG concentrations (data not shown).

\section{DISCUSSION}

This study was specifically designed to examine the effects of plant stanols on serum TAG concentrations in subjects with elevated serum concentrations of TAG. In line with our recent meta-analysis (40), a significant interaction between baseline TAG concentrations and plant stanol intake was found. Furthermore, we have recently shown that plant stanols lowered serum TAG concentrations in subjects with the MS (Plat et al, in press). Based on the meta-analysis (40), the expected decrease in serum TAG concentrations was on average -0.09 $\mathrm{mmol} / \mathrm{L}$ for "borderline high" baseline TAG concentrations [1.7 - $2.2 \mathrm{mmol} / \mathrm{L}$; (149)] and a daily plant stanol intake of $2.5 \mathrm{~g}$. At a similar intake, the expected decline for subjects with "high" baseline TAG values [2.3 - $4.0 \mathrm{mmol} / \mathrm{L}$; (149)], was on average -0.19 $\mathrm{mmol} / \mathrm{L}$. Compared to these predicted effects, our results are slightly different since a reduction in serum TAG concentrations was hardly present at baseline TAG values $<2.3$ $\mathrm{mmol} / \mathrm{L}$. In contrast, at "high" baseline TAG concentration, the average decrease was larger than anticipated, i.e. $-0.35 \mathrm{mmol} / \mathrm{L}$. It is possible that the estimates from the meta-analysis for subjects selected on disturbances in TAG metabolism are less precise, because most of the subjects in the meta-analysis had serum TAG concentrations $<2.0 \mathrm{mmol} / \mathrm{L}$.

As TAG, in the fasted state are mainly transported by VLDL particles, it can be suggested that plant stanols change VLDL metabolism. However, plasma total number of VLDL particles (VLDL-P; including large VLDL-P, medium VLDL-P, small VLDL-P) were not significantly altered after the plant stanol diet. Possibly, the variation in response of the different VLDL particles was too large to reach statistically significant dietary effects. 
Daily consumption of 2.4 g plant stanols also significantly decreased serum LDL cholesterol concentrations by $9.5 \%$. This is in line with the estimated mean change in LDL cholesterol concentrations of $-8.9 \%$ for daily intakes of 2.0 to $2.4 \mathrm{~g}$ plant sterols or stanols (14). Serum apoB-100 concentrations were lowered by $7.1 \%$, which is in the same range as the reductions in total and LDL cholesterol. As expected, no effects on serum HDL cholesterol and apoA-1 concentrations were found. The serum LDL cholesterol-lowering effect was mainly related to a decrease $(-11.2 \%)$ in the atherogenic small LDL-P, but this effect was not statistically significant. A significant positive correlation, however, was found between the plant stanol-induced changes in serum TAG levels and small LDL-P. Small dense LDL-P are particularly considered to be atherogenic, since these particles are retained preferentially by the artery wall and are readily oxidized $(39,153)$. Generation of small, dense LDL occurs by intravascular lipoprotein remodelling as a result of metabolic disturbances, which are frequently present in patients with DM2 and the MS (153). The common underlying predisposing factor may be the development of a fatty liver resulting in hypertriglyceridemia, due to in particular large VLDL-P. Large VLDL-P are a precursor for the synthesis of small dense LDL by LPL mediated processes (154).

Hypertriglyceridemia is also associated with an overproduction of other cardiovascular risk factors, such as glucose, hs-CRP, plasminogen activator inhibitor-1 (PAI-1), fibrinogen, and coagulation factors (39). We therefore looked into the effects of the plant stanol diet on glucose metabolism in our hypertriglyceridemic study population. However, no effects on glucose and insulin concentrations, and the HOMA index were found. These effects agree with earlier findings in healthy subjects, where glucose concentrations were not changed after supplementation of a reduced-calorie orange juice beverage enriched with plant sterols (2 g/d) (136). In addition, consumption of plant sterol $(1.9 \mathrm{~g} / \mathrm{d})$ and plant stanol $(2.0$ $\mathrm{g} / \mathrm{d}$ )-enriched spreads for 10 weeks did not change plasma glucose and serum insulin concentrations in hypercholesterolemic subjects (155).

As plant stanol consumption interferes with intestinal cholesterol absorption, effects on postprandial lipid metabolism may even be more pronounced. Studies on the postprandial effects of phytosterols are however scarce. In healthy subjects, 2-week plant stanol consumption ( $3 \mathrm{~g} / \mathrm{d}$ plant stanols) only tended to diminish postprandial TAG concentrations (156). In another study, the use of plant stanol (3 g/d)-enriched margarine did not decrease postprandial hypertriglyceridemia in statin patients (157).

To summarize, consumption of plant stanols significantly lowered serum LDL cholesterol concentrations in subjects with elevated TAG concentrations, primarily by a decrease in the atherogenic small LDL-P. The plant stanol-induced TAG-lowering in these subjects was related to their baseline TAG concentrations, and was particularly evident in subjects with high serum TAG concentrations. Taken together, these findings show that functional foods enriched with plant stanols are not only of benefit to lower increased serum LDL choles- 
terol concentrations, but also serum triacylglycerols in subjects with overt hypertriglyceridemia.

\section{ACKNOWLEDGEMENTS}

We thank the study participants for their cooperation and enthusiasm. We also thank the technical and dietary staff from our Department for their support. 



\section{CHAPTER 6}

\section{Oxyphytosterols increase the proportion of severe atherosclerotic lesions in LDLR+/- mice}

Elke Theuwissen, Jogchum Plat, Marion J.J. Gijbels, Inge van der Made, Ronald P. Mensink

Submitted 


\section{ABSTRACT}

Objective Oxysterols may be atherogenic and accordingly play an active role in the pathogenesis of cardiovascular disease (CVD). Similar to cholesterol, phytosterols are susceptible to oxidation. However, it is unknown whether oxyphytosterols are atherogenic.

Study design After a 2-week run-in period, 33 female heterozygous LDL receptor-deficient (LDLR+/-) mice were divided to receive for 35 weeks: a control diet (atherogenic diet), an oxysterol diet (control diet with $0.025 \%$ oxysterols), or an oxyphytosterol diet (control diet with $0.025 \%$ oxyphytosterols).

Results At the end of the experiment, serum cholesterol concentrations did not differ between the treatments. Also cholesterol exposure and lipoprotein profiles were similar. During the experiment, changes in serum cholesterol concentrations did not differ between the treatments, except for weeks 7 and 17 (cholesterol-lowering effect of the oxyphytosterols). A shift toward more severe lesions was found after the oxysterol $(p=0.004)$ and oxyphytosterol $(p=0.011)$ diets compared to the control diet. Lesion severity did not differ between the oxysterol and oxyphytosterol groups. Also lesion size and collagen content were similar.

Conclusion Not only oxysterols, but also oxyphytosterols may promote atherosclerosis in LDLR+/- mice, primarily by a shift toward more severe atherosclerotic lesions. 


\section{INTRODUCTION}

In vitro and animal studies have suggested that oxidized cholesterol (oxysterols) is atherogenic and may accordingly play an active role in the pathogenesis of cardiovascular disease (CVD). In fact, various oxysterols have been detected in human tissues and fluids, including plasma, atherogenic lipoproteins, and atherosclerotic plaques (21). Oxysterols arise in tissues not only as a result of enzymatic (during cholesterol catabolism) or nonenzymatic (in vivo oxidation) oxidation reactions, but also from dietary sources (dairy products, egg, meat, fish, bakery products) (21). In vitro, oxysterols have a negative impact on several aspects of cellular cholesterol homeostasis, such as upregulating cholesterol biosynthesis and enhancing cholesterol esterification. In addition, some oxysterols inhibit cholesterol export from foam cells, impair vascular reactivity, are cytotoxic, and induce apoptosis (21). Animal studies have also shown that oxidized cholesterol in the diet accelerates fatty streak lesion formation $(22,23)$. Although, there is no direct evidence that oxysterols contribute to atherogenesis in humans, these observations suggest that oxysterols can contribute to atherogenesis.

Plant sterols (phytosterols) are found in various lipid sources, such as oils from vegetables, nuts, grains, seeds, wood pulp, and leaves. Functional foods enriched with these plant sterols are gaining a prominent position in dietary guidelines to lower the concentrations of the atherogenic LDL particles (14). Plant sterols are structurally related to cholesterol and the presence of one or more unsaturated bonds makes plant sterols susceptible to oxidation as well. Small amounts of oxidized phytosterols (oxyphytosterols) have been demonstrated in the diet (coffee, fried potatoes, wheat flour, vegetable oils) $(158,159)$ and in serum from sitosterolemic patients (25). Although at lower concentrations, oxyphytosterols have also been found in plasma from healthy volunteers (26). In rats, it has been demonstrated that oxyphytosterols were absorbed from the diet and transported into the lymphatics (27). In addition, in apolipoprotein E (apo E)-deficient mice, dietary oxyphytosterols were absorbed and accumulated in serum, liver, and aorta (27). Further, in vitro studies have shown that oxyphytosterols exhibit cytotoxic effects in cultured macrophages similar to those of oxysterols (28). So far, only one study has examined the effects of an oxyphytosterol diet on atherosclerosis in apo E-deficient mice (27). In that study, dietary oxyphytosterols did not promote the development of atherosclerosis compared to phytosterols. Thus, in vitro and animal studies are contrasting and it is still unclear whether oxyphytosterols are atherogenic, as has been suggested for oxysterols. We therefore decided to investigate the effects of oxysterols and oxyphytosterols on lipid metabolism and atherosclerotic lesion development using heterozygous LDL receptor-deficient (LDLR+/-) mice. 


\section{METHODS}

\section{Animal model}

LDLR+/- mice were bred in our laboratory by mating LDLR-/- males with C57BL6/] (Charles River Laboratories, Wilmington, MA, USA) females. Thirty-six female LDLR+/littermates were used for the experiment. Heterozygous LDLR-deficient mice were chosen to simulate a human model for mild hypercholesterolemia. Female mice were used because they are more responsive to the development of diet-induced atherosclerosis (160). At the age of 5 weeks, these mice were divided over 12 cages, with 3 animals per cage. Mice were housed under standard conditions (temperature $20-23^{\circ} \mathrm{C}$, normal day/night rhythm) in wire-topped Macrolon type-I cages with a layer of maize fibers as bedding. All diets and water were given ad libitum. Before the start of the study, mice were fed a regular mouse diet (Hope Farms, Woerden, The Netherlands). The research was conducted in conformity with the Public Health Service Policy on Human Care and Use of Laboratory Animals. The study was approved by The Animal Ethics Committee of Maastricht University.

\section{Test diets and experimental protocol}

At the age of 8 weeks, a run-in period of 2 weeks started, during which all animals received an atherogenic control diet (Table 1). This control diet contained 17.2 percent of energy (en\%) fat with a western-like fatty acid profile (4.5 en\% palm oil, 1.7 en $\%$ coconut oil, 4.0 en $\%$ soy oil, 2.2 en $\%$ olive oil, and 4.8 en\% cacao oil), 47.1 en\% carbohydrate (37.1 en $\%$ sucrose and 10.0 en $\%$ corn starch), 20.0 en $\%$ protein (casein), 0.25 en\% cholesterol, 0.25 en $\%$ cholic acid, 0.20 en $\%$ methionine, 4.85 en $\%$ mineral mixture, and $0.25 \mathrm{en} \%$ vitamin mixture. Thereafter, the 12 cages were randomly allocated to one of the 3 semi-synthetic test diets. For the next 35 weeks, one group $(n=12)$ was fed the atherogenic control diet. Three animals from this group died in weeks 2,16 , and 17 , respectively. The $1^{\text {st }}$ animal died from hydrocefalie, the $2^{\text {nd }}$ from overgrown teeth, and the $3^{\text {rd }}$ for unknown reasons. Results of the control group therefore refer to 9 animals. A second group $(n=12)$ received the same atherogenic control diet except that $10 \%$ of the added cholesterol was replaced by oxysterols $(0.025 \%)$. A third group $(n=12)$ was given the same atherogenic diet, but now with $0.025 \%$ oxyphytosterols replacing cholesterol. As a result, daily intake of oxy(phyto)sterols mimicked daily intake of these oxidation products from the human Western diet. 
TABLE 1: Design of the study ${ }^{1}$.

\begin{tabular}{|c|c|}
\hline \multirow{3}{*}{$\begin{array}{c}\text { Western diet } \\
+0.25 \% \text { cholesterol }+0.25 \% \text { cholate } \\
\mathrm{n}=36(12 \text { cages with } 3 \text { animals })\end{array}$} & $\begin{array}{c}\text { Western diet } \\
+0.25 \% \text { cholesterol }+0.25 \% \text { cholate } \\
n=9^{*}(4 \text { cages with } 3 \text { animals })\end{array}$ \\
\hline & $\begin{array}{c}\text { Western diet } \\
+0.225 \% \text { cholesterol }+0.25 \% \text { cholate } \\
+0.025 \% \text { oxysterols } \\
\mathrm{n}=12(4 \text { cages with } 3 \text { animals })\end{array}$ \\
\hline & $\begin{array}{c}\text { Western diet } \\
+0.225 \% \text { cholesterol }+0.25 \% \text { cholate } \\
+0.025 \% \text { oxyphytosterols } \\
\mathrm{n}=12(4 \text { cages with } 3 \text { animals })\end{array}$ \\
\hline & 27 \\
\hline
\end{tabular}

'The study had a randomized, controlled, parallel design. After a 2-week run-in period, 33 female LDLR+/- mice were divided to receive for 35 weeks: a control diet (atherogenic diet), an oxysterol diet (control diet $+0.025 \%$ oxysterols), or an oxyphytosterol diet (control diet $+0.025 \%$ oxyphytosterols). ${ }^{*}$ Three animals receiving the control diet died in weeks 2,16 , and 17 , respectively.

Oxysterols and oxyphytosterols were prepared by heating cholesterol/sterols at $180^{\circ} \mathrm{C}$ for 3 h (Raisio, Finland). A minor fraction of the identified sterols was still present in nonoxidized form; $4.1 \%$ and $6.4 \%$ for cholesterol and phytosterols, respectively. Given the low amounts of the oxysterol and oxyphytosterol mixtures supplied in the diets, these amounts are negligible and may not interfere with cholesterol metabolism. The composition of the oxysterols and oxyphytosterols is shown in Table 2. Diets were stored at $-20^{\circ} \mathrm{C}$.

TABLE 2: Composition of the oxysterol and oxyphytosterol mixtures'.

\begin{tabular}{lcc}
\hline & Oxysterol mixture & Oxyphytosterol mixture \\
\hline 7-Keto (\% of total) & 40.3 & 28.2 \\
Epoxides (\% of total) & 35.7 & 20.0 \\
$5 \alpha, 6 \alpha$-epoxy- & 30.3 & 7.2 \\
$5 \beta, 6 \beta$-epoxy- & 5.4 & 12.7 \\
Hydroxides (\% of total) & 24.0 & 51.8 \\
$7 \alpha$-hydroxy- & 4.0 & 19.8 \\
$7 \beta$-hydroxy- & 13.8 & 32.0 \\
others & 6.1 & 0.0 \\
\hline
\end{tabular}

${ }^{1}$ Oxysterols and oxyphytosterols were prepared by heating cholesterol/sterols at $180^{\circ} \mathrm{C}$ for 3 hours. 
Blood sampling and body weight

Fasting blood samples were collected 5 times (weeks 2, 7, 17, 27, and 37) by orbital puncture. Blood was collected into plastic tubes (Eppendorf AG, Hamburg, Germany) which were filled with glass beads to prepare serum. Animals were weighed before blood sampling. At the end of the study, all animals were sacrificed after blood sampling. The entire hearts (with aorta) of each mouse were dissected, sectioned perpendicularly to the heart axis just below the atrial tips, and directly placed in aluminum cryotubes (Omnilabo International, Breda, The Netherlands) sited in dry-ice cooled isopentane. The cryotubes were stored at $-80^{\circ} \mathrm{C}$ until use.

\section{Serum cholesterol and lipoprotein profiles}

All blood samples were analyzed directly after sampling for serum total cholesterol using a commercially available enzymatic kit (CHOD/PAP method, Roche Diagnostics, Basel, Switzerland). The area under the curve (AUC) of total cholesterol was calculated for each animal as an indicator of overall cholesterol exposure of the aorta during the 37 week experiment (22). Lipoprotein profiles were made at the end of the intervention period (week 37) by means of fast protein liquid chromatography (FPLC) as described (25). Serum of mice housed in the same cage was pooled. All FPLC fractions of the pooled serum were analyzed for total cholesterol concentrations. Subsequently, these fractions were averaged for each of the 3 diet groups and compared.

\section{Atherosclerotic lesion size and severity}

After storage and before sectioning, the hearts were first placed on an object holder in the microtome/cryostat with the sectioned plane facing up, and were subsequently embedded in Tissue Tek (Sakura Finetek Europe BV, Zoeterwoude, The Netherlands). In this way, the frozen hearts were sectioned toward the aortic axis. Upon reaching the aortic root, serial 7 $\mu \mathrm{m}$ sections were taken until the aortic valves disappeared. These sections were mounted onto microscope slides, and then air-dried for 24 hours on silica. The slides were kept frozen at $-80^{\circ} \mathrm{C}$ until staining.

Atherosclerotic lesion size was analyzed using four serial sections with a $42 \mu \mathrm{m}$ interval per animal. The collected sections were stained with toluidine blue, and digitally photographed (Nikon DXM1200) and quantified using digital image software (Adobe Photoshop CS3). Also lesion severity was quantified, and three types of lesions were discerned: (1) mild lesions: fatty streaks containing only foam cells, (2) moderate lesions: characterized by 
the additional presence of a collagenous cap, and (3) severe lesions: involvement of the media and increased fibrosis, cholesterol clefts, and/or necrosis of the plaque. After scoring each mouse individually, the percentages of lesions classified as mild, moderate, and severe were calculated for each diet group. All analyses were performed by our experimental pathologist, who was blinded with respect to the design of the study.

\section{Atherosclerotic lesion collagen content}

Sirius red staining was performed to visualize collagen content. One section was used per animal. The stained sections were all photographed under the same conditions with a digital microscope camera (Nikon DXM1200). To quantify the percentage of collagen within the lesions, the lesion area was selected manually and a color range selection was applied to measure collagen content (Adobe Photoshop CS3). The same color settings were used for all sections. All analyses were performed by the same technician, who was blinded with respect to the design of the study.

\section{Statistical analyses}

For each animal, responses to the diet were calculated for body weight and serum total cholesterol as the changes between values at the end of the run-in period and the end of the intervention period. Differences between diet groups in body weight, serum total cholesterol concentrations, cholesterol exposure, lipoprotein profiles, atherosclerotic lesion size, and in collagen content were tested for statistical significance by the non-parametric Kruskal-Wallis test. When a significant diet effect was found, the 3 treatments were compared pairwise using a Mann-Whitney test. Values are presented as medians with ranges. Differences in atherosclerotic lesion severity were tested with the Chi-square test. Values are presented as means \pm standard deviation (SD). Differences were considered statistically significant at $\mathrm{p}<0.017$. Statistical analyses were performed using SPSS 11.0 (version 11.0.3) for MacIntosh OS X (version 10.3.9). 


\section{RESULTS}

Body weight, serum cholesterol, and lipoprotein profiles

At the end of the experiment, changes in body weight ( $p=0.867$; Table 3 ) and in serum total cholesterol concentrations ( $p=0.843$; Table 3) were similar for all 3 treatments. There were no differences in AUC of total cholesterol between the 3 animal groups $(p=0.163$;

Table 3), implying that overall cholesterol exposure of the aorta was similar for all 3 groups. Also lipoprotein profiles did not differ between the 3 diets (data not shown).

TABLE 3: Effects of oxysterols and oxyphytosterols on body weight, serum total cholesterol concentrations, cholesterol exposure, and collagen content ${ }^{1}$.

\begin{tabular}{lccc}
\hline & Control & Oxysterols & Oxyphytosterols \\
\hline Body weight $(\mathrm{g})^{2}$ & 23.9 & 23.4 & 23.9 \\
& $(22.5-24.2)$ & $(21.4-27.9)$ & $(22.8-27.4)$ \\
Total cholesterol $(\mathrm{mmol} / \mathrm{L})^{2}$ & 11.8 & 11.0 & 12.1 \\
& $(9.6-16.1)$ & $(9.8-16.7)$ & $(9.5-13.6)$ \\
Cholesterol exposure $(\mathrm{mmol} / \mathrm{L} \times \mathrm{wk})$ & 450 & 405 & 384 \\
& $(341-465)$ & $(323-494)$ & $(329-448)$ \\
Collagen content $\left(10^{3} \mathrm{~mm}^{2}\right)^{2}$ & 117 & 149 & $(7-268)$ \\
Collagen content $(\% \text { of lesion size })^{2}$ & $(22-194)$ & $(39-307)$ & 67 \\
& 52 & 55 & $(11-75)$ \\
\hline
\end{tabular}

${ }^{1}$ All values are medians (with ranges); $\mathrm{n}=33$.

${ }^{2}$ Body weight, serum total cholesterol concentrations, and collagen content are from week 37 of the experiment. 
During the experiment, changes in serum total cholesterol concentrations did not differ between the 3 diet groups, except for weeks 7 and 17. Compared to the control diet, the oxyphytosterol diet significantly lowered serum total cholesterol levels at week 7 by $13 \%$ ( $p=0.006$; Figure 1) and at week 17 by $12 \%(p=0.001$; Figure 1). Serum total cholesterol concentrations in the oxysterol group followed the same pattern as observed for the oxyphytosterols, but the concentrations at weeks 7 and 17 did not differ significantly from those in the control group.

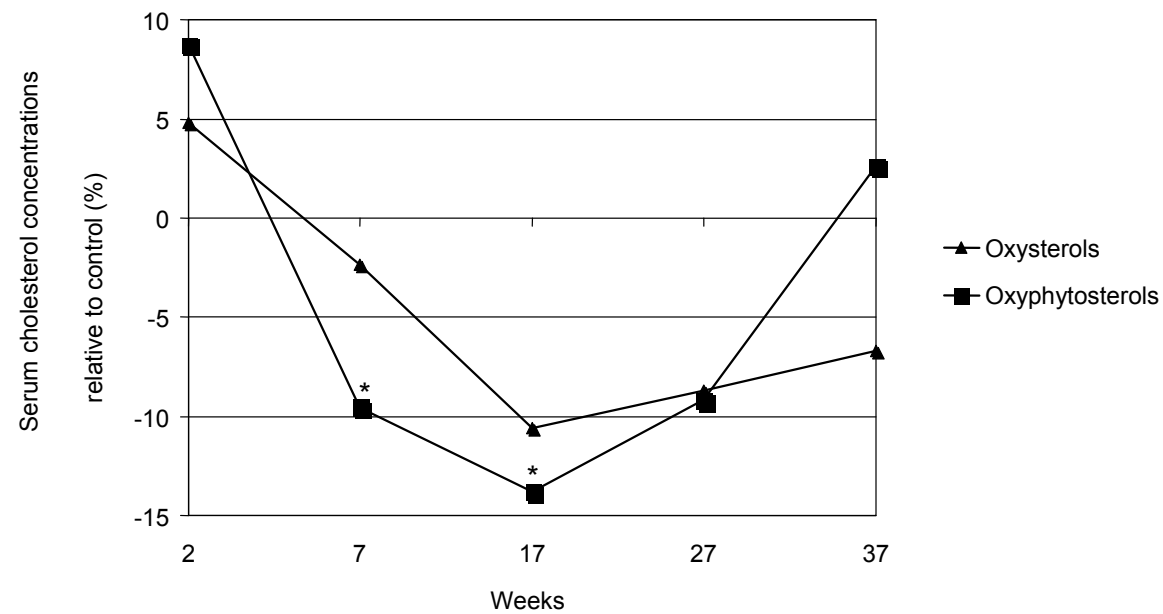

FIGURE 1: Time course of serum cholesterol.

Data are presented as the median of the \% changes relative to the control group; $n=33$. Changes in serum cholesterol were tested for statistical significance by the Kruskal-Wallis test. Pair-wise comparisons were performed by the Mann-Whitney test. ${ }^{*} \mathrm{p}<0.05$ vs. control. 
Atherosclerotic lesion size and severity

Lesion size did not differ between the 3 animal groups after 35 weeks of feeding the control, oxysterol, or oxyphytosterol diets ( $p=0.420$; Table 3). Nevertheless, the atherosclerotic lesion severity pattern differed significantly between the oxysterol and control groups $(p=0.004)$, and the oxyphytosterol and control groups $(p=0.011)$. Compared to the control diet, a shift towards more severe lesions was observed after dietary intake of oxysterols and oxyphytosterols (Figures 2 and 3). No difference was found between the oxysterol and oxyphytosterol groups $(\mathrm{p}=0.125)$.

A

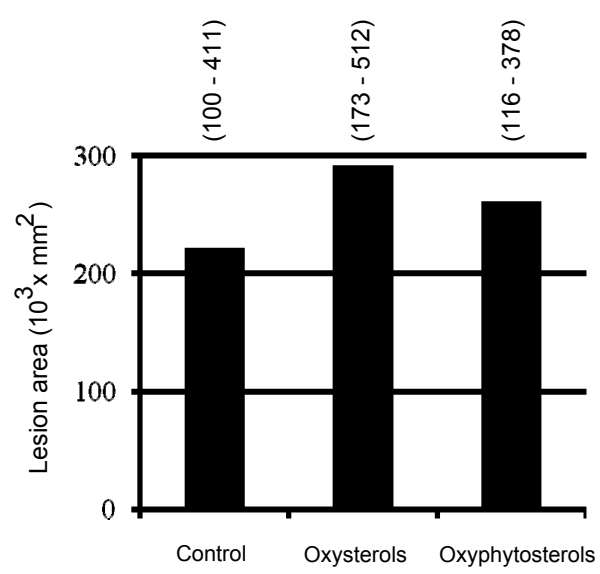

B

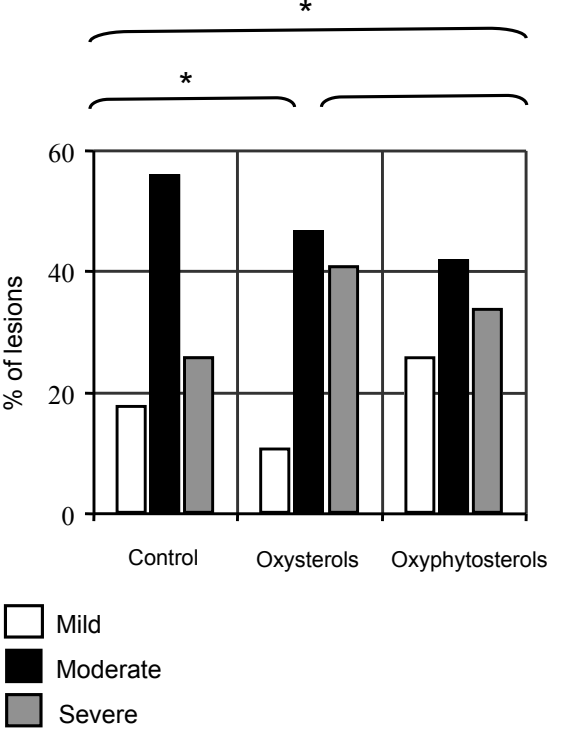

FIGURE 2: Lesion size and severity.

(A) Lesion area $\left(10^{3} \times \mathrm{mm}^{2}\right)$ in LDLR+/- mice. Values are presented as medians with ranges; $\mathrm{n}=33$. (B) Lesion severity (mean \% of total lesions). Lesions were categorized as mild, moderate, and severe. Differences in atherosclerotic lesion severity were tested with the Chi-square test. Values are presented as means \pm SD; $n=33$. ${ }^{*} \mathrm{p}<0.05$ vs. control. 
$1 \mathrm{~A}$
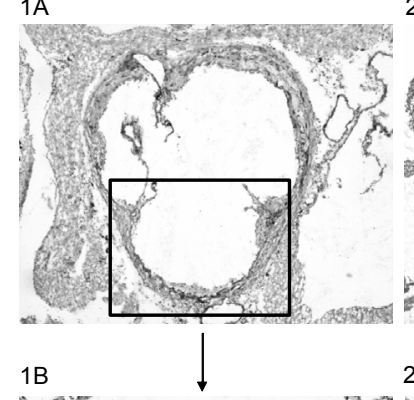

1B

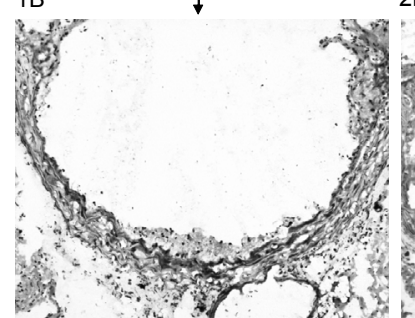

$2 \mathrm{~A}$
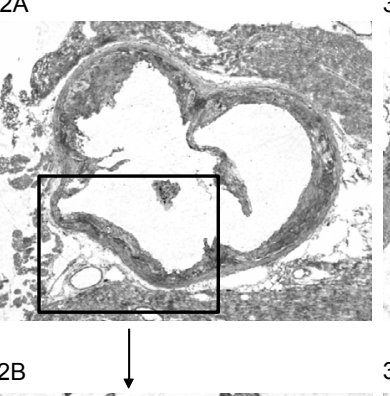

$3 \mathrm{~A}$

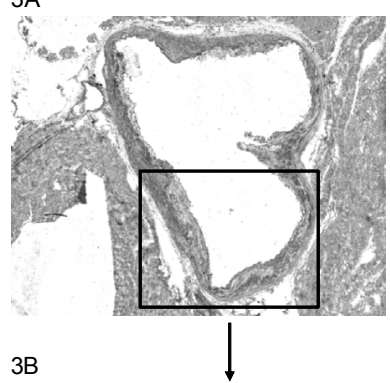

3B

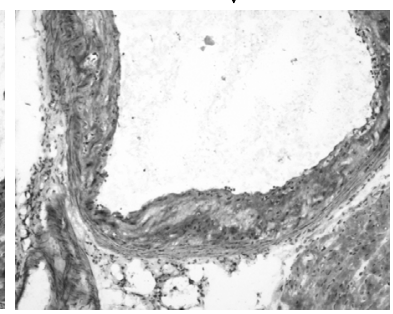

FIGURE 3: Atherosclerotic lesion severity.

(A) Representative sections of the aortic valve area (toluidine blue staining) are presented per diet group (40x). (B) One valve from each section is enlarged (100x). (1A) Section of the aortic valve area of a control animal. (1B) Mild lesion: characterized by only foam cells. (2A) Section of the aortic valve area of an animal from the oxysterol group. (2B) Severe lesion: characterized by foam cells, fibrosis, and necrosis of the plaque. (3A) Section of the aortic valve area of an animal from the oxyphytosterol group. (3B) Severe lesion: characterized by foam cells, fibrosis, and necrosis of the plaque.

\section{Atherosclerotic lesion collagen content}

Collagen content did not differ between the treatments $(p=0.377)$. Also relative collagen content ( $\%$ of lesion size) was the same $(p=0.675)$, indicating that lesion stability was comparable for all 3 diet groups.

\section{DISCUSSION}

In the present study, we found that consumption of oxysterols as well as of oxyphytosterols shifted the atherosclerotic lesion severity pattern toward more severe lesions in female LDLR+/- mice. However, no statistically significant differences in atherosclerotic lesion size were found between any of the diet groups. It is possible that this was due to the duration 
of our study, which might have been too long to observe differences between the diets in lesion size, but not in lesion severity. Also collagen content was the same for the diet groups, implying that lesion stability, contrary to lesion severity, was not differently affected by consumption of the oxysterols and oxyphytosterols.

Only a few studies have examined the atherosclerotic potential of oxysterols or oxyphytosterols. Staprans et al. (23) found significant increases in lesion size after 4 months when comparing a cholesterol with an oxysterol diet in apoE-deficient mice $(n=12)$. These effects as such were not strain-dependent, as after 7 months of feeding, an oxysterol diet also significantly increased lesion size in homozygous LDLR-deficient mice ( $n=12)$. On the other hand, Ando et al. (161) observed no significant differences in lesion size after 8 weeks of feeding a control, cholesterol, or oxysterol diet to apoE-deficient mice $(n=15)$. Nevertheless, the lack of effect may have been related to the relatively short duration of the study. So far, only one study has examined the atherogenicity of oxyphytosterols. In that study, no significant differences were found in lesion size after 9 weeks of feeding a phytosterol or oxyphytosterol diet to apoE-deficient mice $(n=14)(27)$. It should be noted, however, that the effects of oxyphytosterols were compared to those of phytosterols and not to those of an atherogenic control diet, making it difficult to compare these results to our findings. In addition, the duration of that study was substantially shorter than that of our study.

There are several potential mechanisms by which oxy(phyto)sterols may promote the development of atherosclerosis. Oxysterols are cytotoxic for many different cell types, such as endothelial cells (162), macrophages (163), smooth muscle cells (SMC) (164), and lymphocytes (165). Also oxyphytosterols are cytotoxic for cultured macrophages (28). In addition, animal studies have reported that oxysterols induce endothelial injury in vivo (166, 167). Finally, oxyphytosterols increased monocyte chemotactic protein-1 (MCP1, biomarker for endothelial dysfunction) production by endothelial cells (HCAEC; human coronary artery endothelial cells) in vitro (Plat et al., unpublished results). Thus, negative effects of oxy(phyto)sterols on endothelial function may be one of the mechanisms by which dietary oxy(phyto)sterols could promote atherosclerosis. Oxy(phyto)sterols may also accelerate atherosclerosis by inducing foam cell formation (168), affecting proteins involved in lipoprotein metabolism $(169,170)$, or stimulating the expression of cytokines involved in atherogenesis $(171,172)$. Clearly, more research is needed to elucidate the potential atherogenic mechanism of oxy(phyto)sterols.

The observed shift toward more severe lesions was not paralleled by differences in serum total cholesterol levels (at the end of the experiment), cholesterol exposure, or lipoprotein profiles. Consumption of oxyphytosterols, however, resulted in a significant reduction in serum total cholesterol at weeks 7 and 17 . Oxysterol consumption resulted in the same pattern, but differences never reached statistical significance. Staprans et al. (23) observed no significant differences in serum total cholesterol between the control $(18.6 \pm 0.9$ 
$\mathrm{mmol} / \mathrm{L}$; mean $\pm \mathrm{SEM})$ and oxysterol $(18.8 \pm 1.2 \mathrm{mmol} / \mathrm{L})$ diets in the apoE-deficient mice after 4 months. However, serum total cholesterol was significantly reduced by the oxysterol diet $(14.4 \pm 1.4 \mathrm{mmol} / \mathrm{L})$ compared to the control diet $(19.4 \pm 2.1 \mathrm{mmol} / \mathrm{L})$ in the homozygous LDLR-deficient mice after 7 months of feeding (23). Also in the study by Ando et al. (161), no significant differences were observed in serum total cholesterol after 8 weeks of feeding the control $(15.3 \pm 1.4 \mathrm{mmol} / \mathrm{L}$; mean $\pm \mathrm{SEM})$, cholesterol $(16.9 \pm 1.6 \mathrm{mmol} / \mathrm{L})$, or oxysterol $(17.5 \pm 0.3 \mathrm{mmol} / \mathrm{L})$ diets to the apoE-deficient mice. Further, no significant differences were found in serum cholesterol of the apoE-deficient mice after 9 weeks of feeding the phytosterol $(13.7 \pm 1.1 \mathrm{mmol} / \mathrm{L}$; mean $\pm \mathrm{SEM})$ or oxyphytosterol $(16.0 \pm 1.9$ $\mathrm{mmol} / \mathrm{L}$ ) diets (27). However, a notable difference between these findings is the applied dose of oxy(phyto)sterols. It could be that the administered dose in our study was too low to achieve any significant reductions, as serum cholesterol concentrations were (consistently) lower, but not significantly, after consumption of oxy(phyto)sterols. There is no obvious explanation for the differences in the extent of cholesterol lowering at the different points in time.

In this study, daily intake of oxy(phyto)sterols mimicked daily intake of these oxidation products from the human Western diet $(\approx 1 \%$ of dietary cholesterol is oxidized). Results from animal studies suggest that an increased dietary intake of oxyphytosterols, similarly to phytosterols, can elevate their serum concentrations (27). Data regarding the relative absorption of oxyphytosterols are, however, limited. Tomoyori et al. (27) observed a greater total lymph recovery of both oxidized campesterol $(15.9 \%)$ and -sitosterol $(9.1 \%)$ compared to campesterol (5.5\%) and -sitosterol $(2.2 \%)$ in lymph duct-cannulated rats fed cholesterol, phytosterols, or their oxidized derivatives. There is, however, no direct evidence that an increased consumption of oxyphytosterols increases their circulating and tissue concentrations in humans. Various food products, such as dairy products, eggs, meat, and fish are an exogenous source of oxysterols (21). In general, food processing, especially heat treatment, drying, and storage can also induce oxidation. Oxyphytosterols have been identified in coffee beans (173), French fries (174), heated vegetable oils (174), infant milk formulas (175), parenteral nutrition (25), and potato chips (176). Also phytosterol-enriched foods may be a minor dietary source of oxyphytosterols (159). The dietary intake of oxyphytosterols from phytosterol-enriched foods or supplements is estimated to be relatively low $(\leq 1.7 \mathrm{mg} / \mathrm{d})$. Except from dietary sources, like oxysterols, oxyphytosterols present in tissues can also be derived from endogenous formation (177). An important question is whether in vivo formed oxysterols and oxyphytosterols could have the same atherogenic potential as their diet-derived counterparts. The extent to what oxyphytosterols are formed and metabolized in the human body is however not clear yet.

In summary, the present results indicate that not only dietary oxysterols but also dietary oxyphytosterols can promote the development of atherosclerosis in LDLR+/- mice, 
primarily by a shift toward more severe atherosclerotic lesions. To what extent these results can be extrapolated to the human situation, warrants further investigation.

\section{ACKNOWLEDGEMENTS}

We would like to thank the technical staff from our Departments for their support. We also thank Raisio, Finland for kindly providing the oxy(phyto)sterols used to prepare the diets. In particular, we would like to thank Paivi Laakso from the Raisio Group for preparing the oxy(phyto)sterol mixtures.

This study was financially supported by the Netherlands Organization for Health Research and Development (Program Nutrition: Health, Safety and Sustainability; Grant 014-12010). 
CHAPTER 7

\section{GENERAL DISCUSSION}




\section{Introduction}

Cardiovascular disease (CVD) is the number one cause of death globally and is expected to remain in this position for the coming years. This high risk in CVD reflects unhealthy dietary habits, physical inactivity, and tobacco consumption as a result of industrialization, urbanization, economic developments, and food market globalization. For the past 20 years, the focus of public health strategies for reducing the risk of CVD has been lowering serum LDL cholesterol levels. However, recent findings have highlighted not only elevated LDL cholesterol, but also elevated triacylglycerol (TAG) levels as an important risk factor for CVD (39). Also low-grade systemic inflammation is emerging as a novel risk factor, as atherosclerosis is in part due to the accumulation of lipids and inflammatory factors within the vessel wall (178).

Dietary changes are a basic step in managing CVD risk. Functional foods especially developed to lower the cholesterol concentration in the atherogenic LDL particles, and therefore the risk for CVD, are gaining a prominent position in dietary guidelines (9). Such foods contain one or more functional dietary components and therefore provide positive health effects beyond their traditional nutritional value. A dietary strategy with multiple dietary components to target a single CVD risk factor may achieve cumulative effects on that risk factor. Next to this, a dietary strategy that targets multiple CVD risk factors may have a greater impact than a single risk factor-intervention strategy. For example, omega-3 fish-oil fatty acids may exert antithrombotic (179) and hypotriglyceridemic (180) effects.

Within this context, we investigated the role of (combining) different dietary components, such as oat $\beta$-glucan, plant stanols, and oxy(phyto)sterols in managing CVD risk.

\section{Role of oat $\beta$-glucan in managing CVD risk}

\section{Management of hypercholesterolemia}

Oat grains are an important source of dietary fiber, especially the viscous soluble fiber $\beta$ glucan, minerals, and other nutrients, and are becoming increasingly known for their health benefits (181). Daily ingestion of modest quantities of oat $\beta$-glucan can help lowering serum LDL cholesterol levels, thereby reducing the risk of CVD. In our study with slightly hypercholesterolemic volunteers (Chapter 3), daily consumption of muesli enriched with 5 g oat $\beta$-glucan significantly decreased serum LDL cholesterol concentrations with $0.21 \mathrm{mmol} / \mathrm{L}$, in agreement with the predicted reduction of $0.18 \mathrm{mmol} / \mathrm{L}(59)$.

Based on numerous clinical studies, the US Food and Drug Administration (FDA) permitted the use of a claim that viscous soluble fiber from oats has the ability to reduce the risk of 
CVD. The required dose of oat $\beta$-glucan for a single food serving was set at $0.75 \mathrm{~g}$ (daily total intake $\geq 3 \mathrm{~g}$ ) (10). This recommendation is supported by the results of studies that demonstrated significant, although variable, reductions in serum LDL cholesterol concentrations after consumption of different oat $\beta$-glucan-containing products $(45,48,60,61$, 182-184). Inconsistencies in the reported effects of oat products may be due to several factors, such as total fiber intake, baseline serum total cholesterol concentrations, mode of administration, and solubility or molecular weight (MW) of the fiber. Also the viscosity of the $\beta$-glucan in the intestinal tract, which is positively related to its solubility in water and $\mathrm{MW}$, may be an important determinant of its cholesterol-lowering effects.

Regardless of its source, $\beta$-glucan is a polysaccharide composed of glucose molecules (185). In yeast and mushrooms, however, the glucose molecules are joined by $\beta-(1 \rightarrow 3)$ and $\beta$ - $(1 \rightarrow 6)$-glycosidic bonds, while oats and barley are comprised of $\beta-(1 \rightarrow 4)-$ and $\beta$ $(1 \rightarrow 3)$-glycosidic bonds. Oats contain around $4 \% \beta$-glucan; 5 to $10 \% \beta$-glucan is present in barley (72). The FDA recently concluded that whole-grain barley and dry milled barley products are also appropriate sources of $\beta$-glucan to claim that they reduce the risk of CVD (11). In contrast to oats and barley, yeast contains up to $85 \%$ of $\beta$-glucan after processing. However, the $\beta-(1 \rightarrow 3,1 \rightarrow 6)$ structure decreases solubility, thereby compromising the ability to build viscosity in the gastrointestinal tract (185). To our knowledge, only one study has examined the cholesterol-lowering properties of yeast, but unfortunately this study lacked a proper control group (77). Information on the hypocholesterolemic effects of $\beta$-glucan from mushrooms is, however, lacking. It would be therefore interesting to investigate into more detail the importance of the molecular structure of $\beta$-glucan by comparing the hypocholesterolemic effects of the different $\beta$-glucan sources.

\section{Management of inflammation}

Inflammation is emerging as a novel risk factor for CVD, as atherosclerosis is in part due to the accumulation of inflammatory factors within the vessel wall (178). Whether direct targeting of these inflammatory components within the vessel wall by dietary changes also lowers the risk for CVD is difficult to say, since most dietary interventions so far have aimed at lowering serum LDL cholesterol levels. Nevertheless, indirect evidence has been provided by animal studies. For example, mice lacking inflammatory markers, such as the chemokine receptor subtype-2 (CCR2) (186) and the intercellular adhesion molecule-1 (ICAM1) (187) are at decreased risk for atherosclerosis. In humans, infusion of C-reactive protein (CRP) aggravated endothelial dysfunction independent of changes in cholesterol (188). In addition, statins, commonly prescribed lipid-lowering drugs, reduce CRP levels, 
independently of their effects on lipid metabolism (189). Altogether, this suggests that selective targeting of these players in atherogenesis may be useful.

Limited studies, however, have shown that dietary components reduce pro-inflammatory markers. Most studies with fish oil supplementation have shown no effects; conflicting results have been reported with saturated and trans fatty acids, and soy intake. Nevertheless, recent research has demonstrated an association between dietary fiber intake and plasma levels of CRP. A cross-sectional study based on the NHANES cohort, relating total dietary fiber consumption to CRP levels, was one of the first to demonstrate this association (190). In agreement, a 2-year high-fiber Mediterranean-style diet (32 g/d of fiber) significantly lowered CRP levels compared to a prudent diet (17 g/d of fiber) (141). Recently, it has also been shown that 3 -week fiber intake from a diet naturally rich in fiber $(30 \mathrm{~g} / \mathrm{d})$ or a supplement (30 g/d of fiber) lowered CRP levels by $14 \%$ and $18 \%$, respectively, with no difference between these treatment groups (191). Overall, these studies suggest that diets rich in fiber may affect inflammatory parameters However, these studies were not designed to determine the impact of (viscous soluble) fiber alone.

So far, only two studies have examined the effects of viscous soluble fiber on proinflammatory parameters. In a randomized, controlled trial, 6-week supplementation with oat $\beta$-glucan ( $6 \mathrm{~g} / \mathrm{d}$ ) did not change plasma CRP concentrations in hypercholesterolemic subjects (61). However, daily (viscous soluble) fiber intake was not documented. In line with these results, we did not find a beneficial effect of 4-week consumption of oat $\beta$ glucan (5 g/d) enriched-muesli on inflammatory parameters related to atherosclerosis and CVD (Chapter 4). In our study, the mean daily total dietary fiber intake was 22 g, including the supplemented oat $\beta$-glucan (Chapter 3). Recently, 3-month psyllium supplementation (7 or $14 \mathrm{~g} / \mathrm{d}$ ) did not reduce CRP levels compared to no supplements in overweight and obese individuals (192). The initial daily dietary fiber consumption of these participants was $14 \mathrm{~g}$. Again, information regarding total fiber intake during the intervention period is lacking. These findings, however, suggest that taking a single-fiber supplement in contrast to a high-fiber diet may not be an effective way to reduce CRP levels. Nevertheless, it is possible that total daily (viscous soluble) fiber intake was too low and/or the intervention period too short to achieve substantial changes in CRP levels. It would therefore be interesting to evaluate the long-term effects of a high dose of viscous soluble fiber.

The mechanism by which a high-fiber diet reduces the inflammatory state associated with CVD is unclear. Increased consumption of high-density and low-quality foods, such as those rich in refined starches, sugar, and unhealthy lipids (saturated and trans fatty acids), and poor in antioxidants and fiber, may cause an activation of the innate immune system, most likely by an excessive production of pro-inflammatory cytokines associated with a reduced production of anti-inflammatory cytokines (193). This imbalance may favor the generation of a pro-inflammatory milieu, which in turn may produce insulin resistance and 
endothelial dysfunction, an early step in atherogenesis, which will lead to an increased risk for the metabolic syndrome (MS), type II diabetes (DM2), and CVD.

Taken together, future research should provide more insight into the nutritional modulation of inflammation by a single-fiber supplement compared to a whole (high-fiber) diet approach. Elucidating the mechanism by which dietary fiber influences inflammation holds the premise to fully understand the association with cardiovascular health.

\section{Role of plant sterols and stanols in managing CVD risk}

\section{Management of hypercholesterolemia}

Food products enriched with plant sterols or stanols (also called phytosterols) can also lower the atherogenic LDL particles (14). Plant sterols and stanols are already part of our everyday diets, but the amounts are often not high enough to have significant cholesterollowering effects. The challenge of incorporating larger amounts of phytosterols into the diet has been overcome by fortifying foods with plant sterols and stanols. The FDA has also authorized the use of health claims on food labels indicating that regular consumption of food products enriched with plant sterol or stanol esters may reduce the risk of CVD (12). In early 2003, FDA expanded the enforcement discretion regarding the use of the interim health claim rule with respect to free plant sterols and stanols, and CVD (13). The required dose for a single food serving phytosterol esters and free phytosterols is $0.65 \mathrm{~g}$ and $0.40 \mathrm{~g}$, respectively.

In our study (Chapter 3), addition of plant stanols [1.5 g plant stanols (as esters)] to the oat $\beta$-glucan enriched muesli significantly lowered LDL cholesterol levels by $4.4 \%$ (compared to muesli enriched with only oat $\beta$-glucan) (63). The reported effects of the plant stanols in our study were calculated as the difference in effects after oat -glucan and oat -glucan plus plant stanol consumption. The fact that we did not include a separate stanol group is an inherent limitation in our study design. Nevertheless, the calculated plant stanolinduced effect is smaller than the estimated mean change of $-8.5 \%$ for daily intakes of 1.5 to $1.9 \mathrm{~g}$ phytosterols (14). Thus, simultaneous consumption of oat $\beta$-glucan may have interfered with the efficacy of the plant stanols. Several studies have, however, concluded that the effects of plant sterols and stanols do not change when subjects consume a recommended diet, usually characterized by a decrease in fat and cholesterol intake (117121). When complex carbohydrates replace fats, an increase in dietary fiber intake can be expected as well. The majority of these studies did unfortunately not document (changes in) the intake of (viscous soluble) fiber. Also a possible effect of the food matrix on the cholesterol-lowering effectiveness of the phytosterols cannot be ruled out. In a randomized, 
incomplete 3-week crossover study (129), consumption of different plant sterol (1.6 g/d)enriched low-fat foods (e.g. milk, yoghurt, bread, and cereals) decreased serum LDL cholesterol concentrations by 5.4 to $15.9 \%$. It should be noted, however, that also other more recent studies with similar intakes of plant sterols and stanols have reported effects less than the estimated mean change of $-8.5 \%$, based on studies published before 2003 $(14,125-127)$. Based on a recent meta-analysis (40), the expected decrease in serum LDL concentrations is on average $5.1 \%$ for elevated baseline $\mathrm{LDL}$ values $(>2.5 \mathrm{mmol} / \mathrm{L})$ and a daily plant stanol intake of $1.5 \mathrm{~g}$. Altogether, the estimated mean reduction of $8.5 \%$ in serum LDL cholesterol concentrations at daily intakes of 1.5 to $1.9 \mathrm{~g}$ of phytosterols may have been overrated. Nevertheless, the $4.4 \%$ change in LDL cholesterol is somewhat less than in other studies at comparable intakes and therefore interactions of the viscous soluble fiber oat $\beta$-glucan or the food matrix on the efficacy of the plant stanols cannot be excluded.

Phytosterols lower LDL cholesterol by preventing cholesterol absorption (18). Plant sterols and stanols are structurally related to cholesterol and are incorporated into mixed micelles in the intestinal tract. If enough phytosterols are consumed, they may displace cholesterol from the mixed micelles. This ultimately results in a reduction of intestinal cholesterol absorption and a higher fecal excretion of cholesterol and its metabolites. However, plant sterols and stanols do not need to be present in the intestinal lumen simultaneously with cholesterol to inhibit its absorption, suggesting that reduced incorporation of cholesterol into mixed micelles is not the only mechanism. Other processes within the enterocytes, like $A B C$ transporter activity may also play a role in lowering cholesterol by transporting absorbed sterols out of the enterocytes back into the lumen. This hypothesis, however, demands further study. A lower absorption of cholesterol triggers peripheral cells to restore cellular cholesterol homeostasis by a compensatory increase in endogenous cholesterol synthesis (19). Additionally, receptor-mediated cholesterol uptake becomes upregulated (19). The overall effect is a reduction in serum LDL cholesterol concentrations. Addition of plant stanols to the oat $\beta$-glucan-enriched muesli further decreased cholesterol absorption and further raised endogenous cholesterol synthesis in mildly hypercholesterolemic subjects, fitting this suggested mechanism (Chapter 3). In agreement, daily consumption of plant stanol-enriched margarine [2.5 g plant stanols (as esters)] significantly influenced cholesterol absorption and synthesis in subjects with elevated serum TAG concentrations

\section{(Chapter 5).}

In these hypertriglyceridemic subjects, the LDL cholesterol-lowering effect was mainly related to a decrease in the number of the atherogenic small-dense LDL particles, but this effect was however not statistically significant. Nevertheless, a significant correlation was found between the plant-stanol induced changes in serum TAG and small LDL particle levels $(r=0.758, p=0.003)$. Large VLDL particles are a precursor for the synthesis of smalldense LDL by LPL-mediated processes (154). Indeed, when the number of large VLDL 
particles decreased, parallel reductions were found in the number of small LDL particles $(r=0.687, p=0.010$; Chapter 5). However, effects on VLDL particles did not reach statistical significance (see next paragraph). Whether these results suggest that consumption of plant stanols may beneficially affect the number of small-dense LDL particles in hypertriglyceridemic subjects by influencing the hepatic production of VLDL particles requires further study.

\section{Management of hypertriglyceridemia}

Recent evidence suggests that elevated serum TAG concentrations [ $>2.3 \mathrm{mmol} / \mathrm{L} ;(149$, 194)], are also associated with an increased risk for CVD (39). A meta-analysis has also indicated that consumption of plant stanols not only lowers serum LDL cholesterol, but also serum TAG concentrations, in particular in subjects with high baseline TAG concentrations (40). The reason that these effects have not been observed in previous studies may have been due to a lack of statistical power, as effects were only marginal in subjects with normal serum TAG concentrations. In line with this meta-analysis, we found a significant interaction between baseline TAG levels and response-to-treatment (Chapter 5). Based on the meta-analysis, the expected decrease in serum TAG concentrations was on average $0.09 \mathrm{mmol} / \mathrm{L}$ for "borderline high" baseline TAG concentrations [1.7 - $2.2 \mathrm{mmol} / \mathrm{L}$ (149)] and a daily plant stanol intake of $2.5 \mathrm{~g}$. At a similar intake, the expected decline in serum TAG concentrations was on average $-0.19 \mathrm{mmol} / \mathrm{L}$ for "high" baseline TAG values $[2.3$ $4.0 \mathrm{mmol} / \mathrm{L}$ (149)]. Compared to these predicted effects, our results were slightly different; the expected reduction in serum TAG concentrations was hardly present at baseline TAG values $<2.3 \mathrm{mmol} / \mathrm{L}$. In contrast, at "high" baseline TAG concentration, the average decrease was larger than anticipated, i.e. $-0.39 \mathrm{mmol} / \mathrm{L}$. It is possible that the estimates from the meta-analysis for subjects selected on disturbances in TAG metabolism are less precise, because most of the subjects in the meta-analysis had serum TAG concentrations $<2.0 \mathrm{mmol} / \mathrm{L}$.

As TAG are mainly transported by VLDL particles, it can be suggested that VLDL metabolism is changed after consumption of plant stanols. However, plasma concentrations of VLDL particles (large, medium, and small) were not significantly altered after consumption of plant stanols (Chapter 5). Possibly, the variation in response was too large to reach statistical significance. Nevertheless, a significant correlation was found between the plant stanol-induced changes in TAG and the number of large VLDL particles. It could be possible that the mean TAG baseline value $(2.6 \mathrm{mmol} / \mathrm{L})$ was still too low to discern any valid changes in VLDL particle levels. 
Management of inflammation

In our study, addition of $1.5 \mathrm{~g}$ plant stanols to the oat $\beta$-glucan-enriched muesli did not change inflammatory parameters related to atherosclerosis and CVD (Chapter 4). Like for oat $\beta$-glucan, limited information exists on the effects of plant sterols and stanols on inflammatory markers in human volunteers. In line with our results, the majority of these studies showed no significant effects on markers of inflammation, such as CRP, soluble adhesion molecules, and monocyte chemotactic protein-1 (MCP1) (131-134). Nevertheless, the combined administration of plant stanols ( $3 \mathrm{~g} / \mathrm{d}$ ) with a statin significantly reduced CRP concentrations as compared to statin-treatment alone (study 1), but no significant effect was found when supplementing hypercholesterolemic patients (with a positive history for CVD) with the same amount of plant stanols (study 2) (135). In another study (134), however, consumption of plant stanols $(2.5 \mathrm{~g} / \mathrm{d})$ as add-on treatment in statin-users did not change inflammation markers. In contrast, a significant reduction in CRP levels was described after consumption of an orange juice enriched with plant sterols $(2 \mathrm{~g} / \mathrm{d})$ in healthy subjects (136). Overall, no clear explanation can be given to explain these inconsistent effects of phytosterols on parameters of inflammation. As mentioned before, however, a single-dietary supplement may not be an effective way to reduce inflammation.

\section{Combining 2 functional food ingredients}

\section{Management of hypercholesterolemia}

Combining two (or more) functional ingredients into one food product or combining the consumption of several dietary ingredients as part of one dietary portfolio, may achieve cumulative changes in serum LDL cholesterol levels. Indeed, 4-week consumption of a dietary portfolio (1.8 g/d plant sterols, $17.9 \mathrm{~g} / \mathrm{d}$ viscous fiber, $39.1 \mathrm{~g} / \mathrm{d}$ soy protein, and 25.6 g/d almonds) lowered cholesterol levels by $20.6 \%$ compared to a control diet (195). However, the study was not designed to determine the impact of the individual dietary factors. In this respect, the combination of viscous soluble fiber and phytosterols in one product may theoretically be interesting, because the underlying mechanisms of these two ingredients are suggested to be different.

In our study (Chapter 3), daily consumption of the combination muesli enriched with oat $\beta$-glucan ( $5 \mathrm{~g}$ ) and plant stanols (1.5 g) lowered serum LDL cholesterol concentrations by $9.6 \%$. However, this reduction was not as much as expected and an interaction with viscous soluble fiber may have played a role. Indeed, oat $\beta$-glucan consumption decreased serum stanol concentrations, and adding plant stanols did not change the serum stanol 
concentrations (Chapter 3). In contrast, 3-week supplementation with either a plant sterol (1.8 g/d)-enriched bar, a glucomannan-enriched bar (10 g/d of viscous soluble fiber derived from konjac root), or a combination bar lowered serum LDL cholesterol concentrations by $5.4 \%, 13.8 \%$, and $21.7 \%$, respectively $(123)$. These findings did not suggest that glucomannan may attenuate the hypocholesterolemic effects of the plant sterols, although the effect of the plant sterols was less than predicted. Nevertheless, 4 week-combined consumption of plant sterols $(2.6 \mathrm{~g} / \mathrm{d})$ and psyllium $(7.7 \mathrm{~g} / \mathrm{d})$, provided by cookies, also decreased serum LDL cholesterol levels below expectations (124). According to literature (14), daily consumption of $>2.5$ g phytosterols lowers serum LDL cholesterol concentrations on average by $-11.3 \%$, which is already higher than the $-9.8 \%$ decrease in LDL cholesterol found after the combination of psyllium and plant sterols. Unfortunately, the separate contribution of the individual dietary components was not evaluated.

A higher intestinal viscosity may have lowered the phytosterol transport into the enterocytes and accordingly their cholesterol-lowering efficacy. Alternatively, an increase in the intestinal viscosity may have affected lipid emulsification by increasing the droplet size of emulsions thereby decreasing the emulsion interface area. This may reduce the rate of lipolysis and possibly also the hydrolysis of phytosterols. It can than be speculated that active free plant sterols and stanols are released later in the intestinal tract, which may affect their efficacy and absorption. However, more studies are needed to confirm this hypothesis. It should be noted, however, that also the LDL cholesterol-reducing effect of a combination therapy of ezetimibe and plant sterols was not significantly greater than that of ezetimibe monotherapy (196). Ezetimibe may also counteract the plant sterol effect by decreasing the phytosterol amount within the enterocytes. In this study as well, combining two cholesterol-absorption inhibitors had hardly any therapeutic benefit compared with a monotherapy in subjects with mild hypercholesterolemia.

\section{Effects on inflammation}

Similar to supplementing with oat $\beta$-glucan or plant stanols alone, the combination of these dietary factors did not influence inflammatory parameters related to atherosclerosis and CVD (Chapter 4). As mentioned before, however, combining several dietary factors, such as in the Mediterranean-style diet (141), a portfolio diet (37), or a prudent diet (143) may be more effective in reducing parameters of inflammation Indeed, consumption of a prudent diet (higher intakes of fruit, vegetables, legumes, fish, poultry, and whole grains) was inversely associated with plasma concentrations of CRP (143). In agreement, 4-week consumption of the dietary portfolio (1.8 g/d plant sterols, $17.9 \mathrm{~g} / \mathrm{d}$ viscous fiber, $39.1 \mathrm{~g} / \mathrm{d}$ soy protein, and $25.6 \mathrm{~g} / \mathrm{d}$ almonds) lowered CRP levels by $18.2 \%$ compared to a control diet (37). As mentioned already, the 2-year consumption of the Mediterranean-style diet 
(whole grains, fruits, vegetables, nuts, and olive oil) significantly lowered CRP levels compared to the control diet (141). These studies were however not designed to determine the impact of the individual dietary factors. Nevertheless, a change in dietary pattern, compared to changing the intake of one (or two) dietary component(s), may be needed to achieve significant effects on markers of inflammation.

\section{"Reverse of the medal"}

\section{Atherogenicity of phytosterols}

Phytosterolemia is an autosomal recessive disorder with an incidence of 1 in 5 million people (15). Phytosterolemia is characterized by markedly increased serum and tissue plant sterol concentrations, which may lead to premature atherosclerosis. Mutations in the ABCG5 and/or ABCG8 genes disturb the reverse transport of phytosterols from enterocytes into the intestinal lumen and the secretion of phytosterols out of hepatocytes into bile. Altogether, this results in increased intestinal phytosterol absorption and a lower biliary excretion, ultimately leading to phytosterolemia. Studies of patients with phytosterolemia have demonstrated increased phytosterol absorption of 16 to $63 \%$. This augmented phytosterol absorption does not appear to interfere with cholesterol absorption. However, phytosterolemia is associated with a marked reduction in cholesterol synthesis. LDLreceptor activity is normal or enhanced, possibly to compensate for the reduced cholesterol production. Bile acid synthesis is reduced as well, which may increase serum cholesterol levels in some patients. The presence of premature atherosclerosis in individuals with phytosterolemia raises the possibility that even mild increases in serum phytosterol concentrations may be atherogenic. This has important implications because phytosterols are increasingly used as cholesterol-lowering agents. It should be noted, however, that consumption of plant sterols and stanols produces only minor increases in their serum concentrations ( 0.01 to $0.05 \mathrm{mmol} / \mathrm{L}$ ).

The possibility that phytosterols promote atherosclerosis has been examined using animal models and several cross-sectional human studies. The majority of animal studies suggest that plant sterols and stanols are not atherogenic (160, 197-200). Recently, however, supplementation with plant sterols impaired endothelial function, aggravated ischemic brain injury, and promoted atherogenesis in mice (201). Also results of human studies examining the atherogenic potential of phytosterols were not consistent (202-207). It is possible that individuals with polymorphisms in the ABCG5 and ABCG8 genes that affect phytosterol absorption are at higher risk because of their ability to over-absorb phytosterols. 
Clearly, more studies are warranted to examine whether increased serum levels of phytosterols increase the risk for atherosclerosis.

\section{Oxyphytosterols: novel risk factor for CVD}

Several novel risk factors for CVD have emerged, such as small-dense LDL particles, hs$\mathrm{CRP}$, homocysteine, and oxysterols (oxidized cholesterol). Oxysterols possess many potent and diverse biological activities in vitro, several of which may implicate a role in the process of atherosclerosis (21). Various food products, such as dairy products, eggs, meat, and fish are an exogenous source of oxysterols. In general, food processing, especially heat treatment, drying, and storage can also induce oxidation. Due to the presence of a double bound in the steroid nucleus, plant sterols may undergo oxidative processes similar to cholesterol, but detailed knowledge is however lacking. Oxyphytosterols (oxidized plant sterols) have been identified in coffee beans, French fries, heated vegetable oils, infant milk formulas, parenteral nutrition, and potato chips (24). Also phytosterol-enriched foods may be a minor dietary source of oxyphytosterols. However, $<0.1 \%$ of oxyphytosterols was found in commercially available plant sterol-enriched spreads (12 to $68 \mu \mathrm{g} / \mathrm{g}$ of spread). This corresponds to a possible daily ingestion of $<1.7 \mathrm{mg}$ of oxyphytosterols for a recommended daily intake of 20 to $25 \mathrm{~g}$ of spread. In addition to food sources, oxyphytosterols were also detected in human plasma. The plasma concentrations of oxyphytosterols found in the circulation (5 to $57 \mathrm{ng} / \mathrm{mL}$ ) were lower or comparable to those of oxysterols (3 to 154 $\mathrm{ng} / \mathrm{mL}$ ). However, there is no direct evidence yet that an increased consumption of oxyphytosterols increases their circulating and tissue concentrations in humans.

Phytosterols $(<5 \%)$ are absorbed in smaller proportion than cholesterol (55 to $60 \%$ ). The ABCG5 and ABCG8 transporters preferentially secrete phytosterols back into the intestinal lumen. It is possible that the secretion of oxyphytosterols is mediated by the same regulatory complex (ABCG5-ABCG8). In that case, a lower absorption of oxyphytosterols compared to oxysterols can be expected as well. However, no direct comparison of oxyphytosterol versus oxysterol absorption has yet been made. Absorption data regarding the comparison of oxyphytosterols versus phytosterols are still conflicting. It would therefore be interesting to compare the relative absorption of cholesterol, phytosterols, oxysterols, and oxyphytosterols.

It is unknown whether oxyphytosterols are atherogenic, as suggested for oxysterols. Different animal models have shown that dietary oxysterols increase atherosclerotic lesion size. In our animal study (Chapter 6), consumption of oxysterols and oxyphytosterols shifted the atherosclerotic lesion severity pattern toward more severe lesions. So far, however, only one study examined the atherogenicity of oxyphytosterols. In that study (27), no significant differences were found in lesion size of apoE-deficient mice after 9 weeks of 
feeding either a phytosterol or an oxyphytosterol diet. It should be noted however that effects of oxyphytosterols were compared to those of phytosterols and not to those of an atherogenic control diet, making it difficult to compare these results to our findings. In addition, the duration of that study was substantially shorter than our study. Except from dietary sources, like oxysterols, oxyphytosterols can also be derived from endogenous formation. An important question is whether in vivo formed oxysterols and oxyphytosterols could have the same atherogenic potential as their diet-derived counterparts. The extent to what oxyphytosterols are formed and metabolized in the human body is however not clear yet.

There are several potential mechanisms by which oxy(phyto)sterols could promote the development of atherosclerosis. Oxysterols are cytotoxic for many different cell types, such as endothelial cells (162), macrophages (163), smooth muscle cells (SMC) (164), and lymphocytes (165). Also oxyphytosterols are cytotoxic for cultured macrophages (28). In addition, animal studies have reported that oxysterols induce endothelial injury in vivo $(164,167)$. Finally, oxyphytosterols increased MCP1, a biomarker for endothelial dysfunction, production by endothelial cells (HCAEC; human coronary artery endothelial cells; Plat et al., unpublished results). Thus, a negative effect of the oxy(phyto)sterols on endothelial function may be a possible mechanism by which dietary oxy(phyto)sterols promote atherosclerosis. Oxy(phyto)sterols may also accelerate atherosclerosis by inducing foam cell formation (168), affecting proteins involved in lipoprotein metabolism (169, 170), or stimulating the expression of cytokines involved in atherosclerosis $(171,172)$.

Taken together, to what extent these results can be extrapolated to the human situation, warrants further investigation. At the present time, however, there is not sufficient evidence to advice against phytosterol supplementation to reduce LDL cholesterol.

\section{CONCLUSION}

Functional foods enriched with the viscous soluble fiber oat $\beta$-glucan and plant stanols are efficient in lowering the number of atherogenic LDL particles, and therefore the risk for CVD. However, the combined intake of oat $\beta$-glucan and plant stanols lowered LDL cholesterol levels below expectations, possibly because of interference of the viscous soluble fiber with the phytosterol efficacy. Recent findings have highlighted not only elevated LDL cholesterol, but also elevated TAG levels as an important risk factor for CVD. Supplementation with plant stanols not only lowered LDL cholesterol, but also serum TAG levels, particularly in subjects with high baseline TAG levels. Modulation of inflammatory markers may also be a valuable step in managing CVD risk, but consumption of oat $\beta$ glucan and plant stanols did not influence markers related to inflammation. Probably, a change in dietary pattern, compared to changing the intake of one (or two) dietary 
component(s), may be needed to achieve significant effects on markers of inflammation. Oxysterols (oxidized cholesterol) also emerged as a novel risk factor for CVD. Phytosterols may undergo similar oxidative processes, and therefore oxyphytosterols (oxidized phytosterols) may have the same atherogenic potential. In our animal study, consumption of oxysterols and oxyphytosterols promoted atherosclerotic lesion development by a shift toward more severe lesions. How these results compare to the human situation, warrants further study. 

REFERENCES 
1. Ross R. Atherosclerosis--an inflammatory disease. N Engl J Med 1999; 340: 115-26

2. Ginsberg HN. Lipoprotein physiology. Endocrinol Metab Clin North Am 1998; 27: 503-19

3. Russell DW. Cholesterol biosynthesis and metabolism. Cardiovasc Drugs Ther 1992; 6: 10310

4. Davis HR, Jr., Zhu LJ, Hoos LM, Tetzloff G, Maguire M, Liu J, et al. Niemann-Pick C1 Like 1 (NPC1L1) is the intestinal phytosterol and cholesterol transporter and a key modulator of whole-body cholesterol homeostasis. J Biol Chem 2004; 279: 33586-92

5. Oram JF, Vaughan AM. ATP-Binding cassette cholesterol transporters and cardiovascular disease. Circ Res 2006; 99: 1031-43

6. Kidambi S, Patel SB. Cholesterol and non-cholesterol sterol transporters: ABCG5, ABCG8 and NPC1L1: a review. Xenobiotica 2008; 38: 1119-39

7. Redinger RN. The coming of age of our understanding of the enterohepatic circulation of bile salts. Am J Surg 2003; 185: 168-72

8. Cohen JC. Contribution of cholesterol 7alpha-hydroxylase to the regulation of lipoprotein metabolism. Curr Opin Lipidol 1999; 10: 303-7

9. Castro IA, Barroso LP, Sinnecker P. Functional foods for coronary heart disease risk reduction: a meta-analysis using a multivariate approach. Am J Clin Nutr 2005; 82: 32-40

10. FDA Talk Paper. FDA allows whole oat foods to make health claim on reducing the risk of heart disease. 21 January 1997.

11. FDA News. FDA Allows Barley Products to Claim Reduction in Risk of Coronary Heart Disease. 23 December 2005.

12. FDA Talk Paper. FDA authorizes new coronary heart disease health claim for plant sterol and plant stanol esters. 5 September 2000.

13. FDA Letter. FDA letter regarding enforcement discretion wih respect to expanded use of an interim health claim rule about plant sterol/stanol esters and reduced risk of coronary heart disease. 14 February 2003.

14. Katan MB, Grundy SM, Jones P, Law M, Miettinen T, Paoletti R. Efficacy and safety of plant stanols and sterols in the management of blood cholesterol levels. Mayo Clin Proc 2003; 78 : 965-78

15. Patel MD, Thompson PD. Phytosterols and vascular disease. Atherosclerosis 2006; 186: $12-$ 9

16. Dean M, Rzhetsky A, Allikmets R. The human ATP-binding cassette (ABC) transporter superfamily. Genome Res 2001; 11: 1156-66

17. Chan YM, Varady KA, Lin Y, Trautwein E, Mensink RP, Plat J, et al. Plasma concentrations of plant sterols: physiology and relationship with coronary heart disease. Nutr Rev 2006; 64: 385-402

18. Plat J, Mensink RP. Plant stanol and sterol esters in the control of blood cholesterol levels: mechanism and safety aspects. Am J Cardiol 2005; 96: 15D-22D

19. Plat J, Mensink RP. Effects of plant stanol esters on LDL receptor protein expression and on LDL receptor and HMG-CoA reductase mRNA expression in mononuclear blood cells of healthy men and women. Faseb J 2002; 16: 258-60

20. Thompson GR, Grundy SM. History and development of plant sterol and stanol esters for cholesterol-lowering purposes. Am J Cardiol 2005; 96: 3D-9D

21. Brown AJ, Jessup W. Oxysterols and atherosclerosis. Atherosclerosis 1999; 142: 1-28

22. Staprans I, Pan XM, Rapp JH, Feingold KR. Oxidized cholesterol in the diet accelerates the development of aortic atherosclerosis in cholesterol-fed rabbits. Arterioscler Thromb Vasc Biol 1998; 18: 977-83

23. Staprans I, Pan XM, Rapp JH, Grunfeld C, Feingold KR. Oxidized cholesterol in the diet accelerates the development of atherosclerosis in LDL receptor- and apolipoprotein Edeficient mice. Arterioscler Thromb Vasc Biol 2000; 20: 708-14

24. Hovenkamp E, Demonty I, Plat J, Lutjohann D, Mensink RP, Trautwein EA. Biological effects of oxidized phytosterols: a review of the current knowledge. Prog Lipid Res 2008; 47: 37-49 
25. Plat J, Brzezinka H, Lutjohann D, Mensink RP, von Bergmann K. Oxidized plant sterols in human serum and lipid infusions as measured by combined gas-liquid chromatographymass spectrometry. J Lipid Res 2001; 42: 2030-8

26. Grandgirard A, Martine L, Demaison L, Cordelet C, Joffre C, Berdeaux O, et al. Oxyphytosterols are present in plasma of healthy human subjects. Br J Nutr 2004; 91: 101-6

27. Tomoyori H, Kawata Y, Higuchi T, Ichi I, Sato H, Sato M, et al. Phytosterol oxidation products are absorbed in the intestinal lymphatics in rats but do not accelerate atherosclerosis in apolipoprotein E-deficient mice. J Nutr 2004; 134: 1690-6

28. Adcox C, Boyd L, Oehrl L, Allen J, Fenner G. Comparative effects of phytosterol oxides and cholesterol oxides in cultured macrophage-derived cell lines. J Agric Food Chem 2001; 49: 2090-5

29. Yun CH, Estrada A, Van Kessel A, Gajadhar AA, Redmond MJ, Laarveld B. beta-(1-->3, 1-$>4$ ) oat glucan enhances resistance to Eimeria vermiformis infection in immunosuppressed mice. Int J Parasitol 1997; 27: 329-37

30. Yun $\mathrm{CH}$, Estrada A, Van Kessel A, Gajadhar AA, Redmond MJ, Laarveld B. Immunomodulatory effects of oat beta-glucan administered intragastrically or parenterally on mice infected with Eimeria vermiformis. Microbiol Immunol 1998; 42: 457-465

31. Estrada A, Yun CH, Van Kessel A, Li B, Hauta S, Laarveld B. Immunomodulatory activities of oat beta-glucan in vitro and in vivo. Microbiol Immunol 1997; 41: 991-998

32. Davis JM, Murphy EA, Brown AS, Carmichael MD, Ghaffar A, Mayer EP. Effects of moderate exercise and beta-glucan on innate immune function and susceptibility to respiratory infection. Am J Physiol Regul Integr Comp Physiol 2004; 286: R366-R372

33. Ramakers JD, Volman JJ, Biorklund M, Onning G, Mensink RP, Plat J. Fecal water from ileostomic patients consuming oat beta-glucan enhances immune responses in enterocytes. Mol Nutr Food Res 2007; 51: 211-20

34. Nashed B, Yeganeh B, HayGlass KT, Moghadasian MH. Antiatherogenic effects of dietary plant sterols are associated with inhibition of proinflammatory cytokine production in Apo E-KO mice. J Nutr 2005; 135: 2438-44

35. Bouic PJD. The role of phytosterols and phytosterolins in immune modulation: a review of the past 10 years. Opin Clin Nutr Metab Care 2001; 4: 471-475

36. Calpe-Berdiel L, Escola-Gil JC, Benitez S, Bancells C, Gonzalez-Sastre F, Palomer X, et al. Dietary phytosterols modulate T-helper immune response but do not induce apparent antiinflammatory effects in a mouse model of acute, aseptic inflammation. Life Sci 2007; 80: 1951-6

37. Jenkins DJ, Kendall CW, Marchie A, Faulkner DA, Wong JM, de Souza R, et al. Effects of a dietary portfolio of cholesterol-lowering foods vs lovastatin on serum lipids and C-reactive protein. Jama 2003; 290: 502-10

38. Koenig W, Sund M, Frohlich M, Fischer HG, Lowel H, Doring A, et al. C-Reactive protein, a sensitive marker of inflammation, predicts future risk of coronary heart disease in initially healthy middle-aged men: results from the MONICA (Monitoring Trends and Determinants in Cardiovascular Disease) Augsburg Cohort Study, 1984 to 1992. Circulation 1999; 99: 237-42

39. Malloy M, Kane J. A risk factor for atherosclerosis: triglyceride-rich lipoproteins. 2001; 47: 111-136

40. Naumann E, Plat J, Kester AD, Mensink RP. The baseline serum lipoprotein profile is related to plant stanol induced changes in serum lipoprotein cholesterol and triacylglycerol concentrations. J Am Coll Nutr 2008; 27: 117-26

41. Anderson JW, Deakins DA, Floore TL, Smith BM, Whitis SE. Dietary fiber and coronary heart disease. Crit Rev Food Sci Nutr 1990; 29: 95-147

42. Bazzano LA, He J, Ogden LG, Loria CM, Whelton PK. Dietary fiber intake and reduced risk of coronary heart disease in US men and women: the National Health and Nutrition Examination Survey I Epidemiologic Follow-up Study. Arch Intern Med 2003; 163: 1897-904 
43. Dikeman CL, Fahey GC. Viscosity as related to dietary fiber: a review. Crit Rev Food Sci Nutr 2006; 46: 649-63

44. De Groot AP, Luyken R, Pikaar NA. Cholesterol-lowering effect of rolled oats. Lancet 1963; 2: $303-4$

45. Braaten JT, Wood PJ, Scott FW, Wolynetz MS, Lowe MK, Bradley-White P, et al. Oat betaglucan reduces blood cholesterol concentration in hypercholesterolemic subjects. Eur J Clin Nutr 1994; 48: 465-74

46. Anderson JW. Dietary fibre, complex carbohydrate and coronary artery disease. Can J Cardiol 1995; 11 Suppl G: 55G-62G

47. Kirby RW, Anderson JW, Sieling B, Rees ED, Chen WJ, Miller RE, et al. Oat-bran intake selectively lowers serum low-density lipoprotein cholesterol concentrations of hypercholesterolemic men. Am J Clin Nutr 1981; 34: 824-9

48. Anderson JW, Spencer DB, Hamilton CC, Smith SF, Tietyen J, Bryant CA, et al. Oat-bran cereal lowers serum total and LDL cholesterol in hypercholesterolemic men. Am J Clin Nutr 1990; 52: 495-9

49. Anderson JW, Gilinsky NH, Deakins DA, Smith SF, O'Neal DS, Dillon DW, et al. Lipid responses of hypercholesterolemic men to oat-bran and wheat-bran intake. Am J Clin Nutr 1991; 54: 678-83

50. Van Horn L, Moag-Stahlberg A, Liu KA, Ballew C, Ruth K, Hughes R, et al. Effects on serum lipids of adding instant oats to usual American diets. Am J Public Health 1991; 81: 183-8

51. Davidson MH, Dugan LD, Burns JH, Bova J, Story K, Drennan KB. The hypocholesterolemic effects of beta-glucan in oatmeal and oat bran. A dose-controlled study. Jama 1991; 265: 1833-9

52. Gerhardt AL, Gallo NB. Full-fat rice bran and oat bran similarly reduce hypercholesterolemia in humans. J Nutr 1998; 128: 865-9

53. Swain J, Rouse I, Curley C, Sacks FM. Comparison of the effects of oat bran and low-fiber wheat on serum lipoprotein levels and blood pressure. N Engl J Med 1990; 322: 147-152

54. Leadbetter J, Ball MJ, Mann JI. Effects of increasing quantities of oat bran in hypercholesterolemic people. Am J Clin Nutr 1991; 54: 841-5

55. Lovegrove JA, Clohessy A, Milon H, Williams CM. Modest doses of beta-glucan do not reduce concentrations of potentially atherogenic lipoproteins. Am J Clin Nutr 2000; 72: 4955

56. Kerckhoffs DA, Hornstra G, Mensink RP. Cholesterol-lowering effect of beta-glucan from oat bran in mildly hypercholesterolemic subjects may decrease when beta-glucan is incorporated into bread and cookies. Am J Clin Nutr 2003; 78: 221-7

57. Ripsin CM, Keenan JM, Jacobs DR, Jr., Elmer PJ, Welch RR, Van Horn L, et al. Oat products and lipid lowering. A meta-analysis. Jama 1992; 267: 3317-25

58. Glore SR, Van Treeck D, Knehans AW, Guild M. Soluble fiber and serum lipids: a literature review. J Am Diet Assoc 1994; 94: 425-36

59. Brown L, Rosner B, Willett WW, Sacks FM. Cholesterol-lowering effects of dietary fiber: a meta-analysis. Am J Clin Nutr 1999; 69: 30-42

60. Onning G, Wallmark A, Persson M, Akesson B, Elmstahl S, Oste R. Consumption of oat milk for 5 weeks lowers serum cholesterol and LDL cholesterol in free-living men with moderate hypercholesterolemia. Ann Nutr Metab 1999; 43: 301-9

61. Queenan KM, Stewart ML, Smith KN, Thomas W, Fulcher RG, Slavin JL. Concentrated oat beta-glucan, a fermentable fiber, lowers serum cholesterol in hypercholesterolemic adults in a randomized controlled trial. Nutr J 2007; 6: 6

62. Reyna-Villasmil N, Bermudez-Pirela V, Mengual-Moreno E, Arias N, Cano-Ponce C, LealGonzalez E, et al. Oat-derived beta-glucan significantly improves HDLC and diminishes LDLC and non-HDL cholesterol in overweight individuals with mild hypercholesterolemia. Am J Ther 2007; 14: 203-12

63. Theuwissen E, Mensink RP. Simultaneous Intake of \{beta\}-Glucan and Plant Stanol Esters Affects Lipid Metabolism in Slightly Hypercholesterolemic Subjects. J Nutr 2007; 137: 583-8 
64. Naumann E, van Rees AB, Onning G, Oste R, Wydra M, Mensink RP. \{beta\}-Glucan incorporated into a fruit drink effectively lowers serum LDL-cholesterol concentrations. Am J Clin Nutr 2006; 83: 601-5

65. Biorklund M, van Rees A, Mensink RP, Onning G. Changes in serum lipids and postprandial glucose and insulin concentrations after consumption of beverages with beta-glucans from oats or barley: a randomised dose-controlled trial. Eur J Clin Nutr 2005; 59: 1272-81

66. Pick ME, Hawrysh ZJ, Gee MI, Toth E, Garg ML, Hardin RT. Oat bran concentrate bread products improve long-term control of diabetes: a pilot study. J Am Diet Assoc 1996; 96: 1254-61

67. Dubois C, Armand M, Ferezou J, Beaumier G, Portugal H, Pauli AM, et al. Postprandial appearance of dietary deuterated cholesterol in the chylomicron fraction and whole plasma in healthy subjects. Am J Clin Nutr 1996; 64: 47-52

68. Lia A, Andersson H, Mekki N, Juhel C, Senft M, Lairon D. Postprandial lipemia in relation to sterol and fat excretion in ileostomy subjects given oat-bran and wheat test meals. Am J Clin Nutr 1997; 66: 357-65

69. Cara L, Dubois C, Borel P, Armand M, Senft M, Portugal H, et al. Effects of oat bran, rice bran, wheat fiber, and wheat germ on postprandial lipemia in healthy adults. Am J Clin Nutr 1992; 55: 81-8

70. Redard CL, Davis PA, Schneeman BO. Dietary fiber and gender: effect on postprandial lipemia. Am J Clin Nutr 1990; 52: 837-45

71. Behall KM, Scholfield DJ, Hallfrisch J. Lipids significantly reduced by diets containing barley in moderately hypercholesterolemic men. J Am Coll Nutr 2004; 23: 55-62

72. Keogh GF, Cooper GJ, Mulvey TB, McArdle BH, Coles GD, Monro JA, et al. Randomized controlled crossover study of the effect of a highly beta-glucan-enriched barley on cardiovascular disease risk factors in mildly hypercholesterolemic men. Am J Clin Nutr 2003; 78: 711-8

73. Behall KM, Scholfield DJ, Hallfrisch J. Diets containing barley significantly reduce lipids in mildly hypercholesterolemic men and women. Am J Clin Nutr 2004; 80: 1185-93

74. Keenan JM, Goulson M, Shamliyan T, Knutson N, Kolberg L, Curry L. The effects of concentrated barley beta-glucan on blood lipids in a population of hypercholesterolaemic men and women. Br J Nutr 2007; 97: 1162-8

75. Truswell AS. Cereal grains and coronary heart disease. Eur J Clin Nutr 2002; 56: 1-14

76. Lupton JR, Robinson MC, Morin JL. Cholesterol-lowering effect of barley bran flour and oil. J Am Diet Assoc 1994; 94: 65-70

77. Nicolosi R, Bell SJ, Bistrian BR, Greenberg I, Forse RA, Blackburn GL. Plasma lipid changes after supplementation with beta-glucan fiber from yeast. Am J Clin Nutr 1999; 70: 208-12

78. Marlett JA, Fischer MH. The active fraction of psyllium seed husk. Proc Nutr Soc 2003; 62: 207-9

79. Garvin JE, Forman DT, Eiseman WR, Phillips CR. Lowering of human serum cholesterol by an oral hydrophilic colloid. Proc Soc Exp Biol Med 1965; 120: 744-6

80. Moreyra AE, Wilson AC, Koraym A. Effect of combining psyllium fiber with simvastatin in lowering cholesterol. Arch Intern Med 2005; 165: 1161-6

81. Ziai SA, Larijani B, Akhoondzadeh S, Fakhrzadeh H, Dastpak A, Bandarian F, et al. Psyllium decreased serum glucose and glycosylated hemoglobin significantly in diabetic outpatients. J Ethnopharmacol 2005; 102: 202-7

82. FDA Talk Paper. FDA allows foods containing psyllium to make health claim on reducing risk of heart disease. 17 February 1998.

83. Thakur BR, Singh RK, Handa AK. Chemistry and uses of pectin--a review. Crit Rev Food Sci Nutr 1997; 37: 47-73

84. Keys A, Grande F, Anderson JT. Fiber and pectin in the diet and serum cholesterol concentration in man. Proc Soc Exp Biol Med 1961; 106: 555-8

85. Schwab U, Louheranta A, Torronen A, Uusitupa M. Impact of sugar beet pectin and polydextrose on fasting and postprandial glycemia and fasting concentrations of serum total 
and lipoprotein lipids in middle-aged subjects with abnormal glucose metabolism. Eur J Clin Nutr 2006; 60: 1073-80

86. Mahalko JR, Sandstead HH, Johnson LK, Inman LF, Milne DB, Warner RC, et al. Effect of consuming fiber from corn bran, soy hulls, or apple powder on glucose tolerance and plasma lipids in type II diabetes. Am J Clin Nutr 1984; 39: 25-34

87. Fahrenbach MJ, Riccardi BA, J.C. S, Lourie IN, Heider JG. Comparative effetcs of guar gum and pectin on human serum cholesterol levels. Circulation 1965; 31: 11

88. Blake DE, Hamblett CJ, Frost PG, Judd PA, Ellis PR. Wheat bread supplemented with depolymerized guar gum reduces the plasma cholesterol concentration in hypercholesterolemic human subjects. Am J Clin Nutr 1997; 65: 107-13

89. Yamatoya K, Kuwano K, Suzuki J. Effects of hydrolyzed guar gum on cholesterol and glucose in humans. Food hydrocolloids 1997; 11: 239-242

90. Eastwood M, Mowbray L. The binding of the components of mixed micelle to dietary fiber. Am J Clin Nutr 1976; 29: 1461-7

91. Kritchevsky D, Story JA. Binding of bile salts in vitro by nonnutritive fiber. J Nutr 1974; 104 458-62

92. Lia A, Hallmans G, Sandberg AS, Sundberg B, Aman P, Andersson H. Oat beta-glucan increases bile acid excretion and a fiber-rich barley fraction increases cholesterol excretion in ileostomy subjects. Am J Clin Nutr 1995; 62: 1245-51

93. Andersson $\mathrm{M}$, Ellegard L, Andersson H. Oat bran stimulates bile acid synthesis within $8 \mathrm{~h}$ as measured by 7alpha-hydroxy-4-cholesten-3-one. Am J Clin Nutr 2002; 76: 1111-6

94. Ellegard L, Andersson H. Oat bran rapidly increases bile acid excretion and bile acid synthesis: an ileostomy study. Eur J Clin Nutr 2007; 61: 938-45

95. Bridges SR, Anderson JW, Deakins DA, Dillon DW, Wood CL. Oat bran increases serum acetate of hypercholesterolemic men. Am J Clin Nutr 1992; 56: 455-9

96. Wolever TM, Fernandes J, Rao AV. Serum acetate:propionate ratio is related to serum cholesterol in men but not women. J Nutr 1996; 126: 2790-7

97. Wolever TM, Brighenti F, Royall D, Jenkins AL, Jenkins DJ. Effect of rectal infusion of short chain fatty acids in human subjects. Am J Gastroenterol 1989; 84: 1027-33

98. Wolever TM, Spadafora PJ, Cunnane SC, Pencharz PB. Propionate inhibits incorporation of colonic [1,2-13C]acetate into plasma lipids in humans. Am J Clin Nutr 1995; 61: 1241-7

99. Wursch P, Pi-Sunyer FX. The role of viscous soluble fiber in the metabolic control of diabetes. A review with special emphasis on cereals rich in beta-glucan. Diabetes Care 1997; 20: 1774-80

100. Trowell H. Ischemic heart disease and dietary fiber. Am J Clin Nutr 1972; 25: 926-32

101. Mozaffarian D, Kumanyika SK, Lemaitre RN, Olson JL, Burke GL, Siscovick DS. Cereal, fruit, and vegetable fiber intake and the risk of cardiovascular disease in elderly individuals. Jama 2003; 289: 1659-66

102. Liu S, Buring JE, Sesso HD, Rimm EB, Willett WC, Manson JE. A prospective study of dietary fiber intake and risk of cardiovascular disease among women. J Am Coll Cardiol 2002; 39: 49-56

103. Wolk A, Manson JE, Stampfer MJ, Colditz GA, Hu FB, Speizer FE, et al. Long-term intake of dietary fiber and decreased risk of coronary heart disease among women. Jama 1999; 281: 1998-2004

104. Rimm EB, Ascherio A, Giovannucci E, Spiegelman D, Stampfer MJ, Willett WC. Vegetable, fruit, and cereal fiber intake and risk of coronary heart disease among men. Jama 1996; 275: 447-51

105. Pietinen P, Rimm EB, Korhonen P, Hartman AM, Willett WC, Albanes D, et al. Intake of dietary fiber and risk of coronary heart disease in a cohort of Finnish men. The AlphaTocopherol, Beta-Carotene Cancer Prevention Study. Circulation 1996; 94: 2720-7

106. FDA Letter. Health claims: fruits, vegetables, and grain products that contain fiber, particularly soluble fiber, and risk of coronary heart disease., 1 April 2002. 
107. Bell S, Goldman VM, Bistrian BR, Arnold AH, Ostroff G, Forse RA. Effect of beta-glucan from oats and yeast on serum lipids. Crit Rev Food Sci Nutr 1999; 39: 189-202

108. Marlett JA, Hosig KB, Vollendorf NW, Shinnick FL, Haack VS, Story JA. Mechanism of serum cholesterol reduction by oat bran. Hepatology 1994; 20: 1450-7

109. Reihner E, Angelin B, Rudling M, Ewerth S, Bjorkhem I, Einarsson K. Regulation of hepatic cholesterol metabolism in humans: stimulatory effects of cholestyramine on HMG-CoA reductase activity and low density lipoprotein receptor expression in gallstone patients. J Lipid Res 1990; 31: 2219-26

110. Plat J, Nichols JA, Mensink RP. Plant sterols and stanols: effects on mixed micellar composition and LXR (target gene) activation. J Lipid Res 2005; 46: 2468-76

111. Plat J, Mensink RP. Effects of diets enriched with two different plant stanol ester mixtures on plasma ubiquinol-10 and fat-soluble antioxidant concentrations. Metabolism 2001; 50: 5209

112. Friedewald WT, Levy RI, Fredrickson DS. Estimation of the concentration of low-density lipoprotein cholesterol in plasma, without use of the preparative ultracentrifuge. Clin Chem 1972; 18: 499-502

113. Hess D, Keller HE, Oberlin B, Bonfanti R, Schuep W. Simultaneous determination of retinol, tocopherols, carotenes and lycopene in plasma by means of high-performance liquid chromatography on reversed phase. Int J Vitam Nutr Res 1991; 61: 232-8

114. Oostenbrug GS, Mensink RP, Hardeman MR, De Vries T, Brouns F, Hornstra G. Exercise performance, red blood cell deformability, and lipid peroxidation: effects of fish oil and vitamin E. J Appl Physiol 1997; 83: 746-52

115. Galman C, Arvidsson I, Angelin B, Rudling M. Monitoring hepatic cholesterol 7alphahydroxylase activity by assay of the stable bile acid intermediate 7alpha-hydroxy-4cholesten-3-one in peripheral blood. J Lipid Res 2003; 44: 859-66

116. Plat J, Kerckhoffs DA, Mensink RP. Therapeutic potential of plant sterols and stanols. Curr Opin Lipidol 2000; 11: 571-6

117. Jones PJ, Ntanios FY, Raeini-Sarjaz M, Vanstone CA. Cholesterol-lowering efficacy of a sitostanol-containing phytosterol mixture with a prudent diet in hyperlipidemic men. Am J Clin Nutr 1999; 69: 1144-50

118. Maki KC, Davidson MH, Umporowicz DM, Schaefer EJ, Dicklin MR, Ingram KA, et al. Lipid responses to plant-sterol-enriched reduced-fat spreads incorporated into a National Cholesterol Education Program Step I diet. Am J Clin Nutr 2001; 74: 33-43

119. Hallikainen MA, Uusitupa MI. Effects of 2 low-fat stanol ester-containing margarines on serum cholesterol concentrations as part of a low-fat diet in hypercholesterolemic subjects. Am J Clin Nutr 1999; 69: 403-10

120. Hallikainen MA, Sarkkinen ES, Gylling H, Erkkila AT, Uusitupa MI. Comparison of the effects of plant sterol ester and plant stanol ester-enriched margarines in lowering serum cholesterol concentrations in hypercholesterolaemic subjects on a low-fat diet. Eur J Clin Nutr 2000; 54: 715-25

121. Cleghorn CL, Skeaff CM, Mann J, Chisholm A. Plant sterol-enriched spread enhances the cholesterol-lowering potential of a fat-reduced diet. Eur J Clin Nutr 2003; 57: 170-6

122. Nigon F, Serfaty-Lacrosniere C, Beucler I, Chauvois D, Neveu C, Giral P, et al. Plant sterolenriched margarine lowers plasma LDL in hyperlipidemic subjects with low cholesterol intake: effect of fibrate treatment. Clin Chem Lab Med 2001; 39: 634-40

123. Yoshida M, Vanstone CA, Parsons WD, Zawistowski J, Jones PJ. Effect of plant sterols and glucomannan on lipids in individuals with and without type II diabetes. Eur J Clin Nutr 2006; 60: 529-37

124. Shrestha S, Volek JS, Udani J, Wood RJ, Greene CM, Aggarwal D, et al. A Combination Therapy Including Psyllium and Plant Sterols Lowers LDL Cholesterol by Modifying Lipoprotein Metabolism in Hypercholesterolemic Individuals. J Nutr 2006; 136: 2492-7 
125. Hallikainen MA, Sarkkinen ES, Uusitupa MI. Plant stanol esters affect serum cholesterol concentrations of hypercholesterolemic men and women in a dose-dependent manner. J Nutr 2000; 130: 767-76

126. Jones PJ, Raeini-Sarjaz M, Ntanios FY, Vanstone CA, Feng JY, Parsons WE. Modulation of plasma lipid levels and cholesterol kinetics by phytosterol versus phytostanol esters. J Lipid Res 2000; 41: 697-705

127. Noakes M, Clifton PM, Doornbos AM, Trautwein EA. Plant sterol ester-enriched milk and yoghurt effectively reduce serum cholesterol in modestly hypercholesterolemic subjects. Eur J Nutr 2004; 44: 214-22

128. O'Neill FH, Brynes A, Mandeno R, Rendell N, Taylor G, Seed M, et al. Comparison of the effects of dietary plant sterol and stanol esters on lipid metabolism. Nutr Metab Cardiovasc Dis 2004; 14: 133-42

129. Clifton PM, Noakes M, Sullivan D, Erichsen N, Ross D, Annison G, et al. Cholesterollowering effects of plant sterol esters differ in milk, yoghurt, bread and cereal. Eur J Clin Nutr 2004; 58: 503-9

130. Pasquier B, Armand M, Castelain C, Guillon F, Borel P, Lafont H, et al. Emulsification and lipolysis of triacylglycerols are altered by viscous soluble dietary fibres in acidic gastric medium in vitro. Biochem J 1996; 314 ( Pt 1): 269-75

131. Kerckhoffs DAJM. Dietary components and cardiovascular risk markers: effects of tocotrienols, beta-glucan and plant stanol esters. Thesis. In.Book Dietary components and cardiovascular risk markers: effects of tocotrienols, beta-glucan and plant stanol esters. Thesis, 2003; Maastricht, Netherlands: Maastricht University.

132. AbuMweis SS, Vanstone CA, Ebine N, Kassis A, Ausman LM, Jones PJ, et al. Intake of a single morning dose of standard and novel plant sterol preparations for 4 weeks does not dramatically affect plasma lipid concentrations in humans. J Nutr 2006; 136: 1012-6

133. Acuff RV, Cai DJ, Dong ZP, Bell D. The lipid lowering effect of plant sterol ester capsules in hypercholesterolemic subjects. Lipids Health Dis 2007; 6: 11

134. De Jong A, Plat J, Bast A, Godschalk RW, Basu S, Mensink RP. Effects of plant sterol and stanol ester consumption on lipid metabolism, antioxidant status and markers of oxidative stress, endothelial function and low-grade inflammation in patients on current statin treatment. Eur J Clin Nutr 2008; 62: 263-73

135. Cater NB, Garcia-Garcia AB, Vega GL, Grundy SM. Responsiveness of plasma lipids and lipoproteins to plant stanol esters. Am J Cardiol 2005; 96: 23D-28D

136. Devaraj S, Autret BC, Jialal I. Reduced-calorie orange juice beverage with plant sterols lowers C-reactive protein concentrations and improves the lipid profile in human volunteers. Am J Clin Nutr 2006; 84: 756-61

137. Inoue $\mathrm{T}$, Komoda $\mathrm{H}$, Nonaka $\mathrm{M}$, Kameda $\mathrm{M}$, Uchida $\mathrm{T}$, Node $\mathrm{K}$. Interleukin-8 as an independent predictor of long-term clinical outcome in patients with coronary artery disease. Int J Cardiol 2008; 124: 319-25

138. Haddy N, Sass C, Droesch S, Zaiou M, Siest G, Ponthieux A, et al. IL-6, TNF-alpha and atherosclerosis risk indicators in a healthy family population: the STANISLAS cohort. Atherosclerosis 2003; 170: 277-83

139. Ramakers JD, Plat J, Sebedio JL, Mensink RP. Effects of the individual isomers cis-9,trans-11 vs. trans-10, cis-12 of conjugated linoleic acid (CLA) on inflammation parameters in moderately overweight subjects with LDL-phenotype B. Lipids 2005; 40: 909-18

140. Han SN, Leka LS, Lichtenstein AH, Ausman LM, Schaefer EJ, Meydani SN. Effect of hydrogenated and saturated, relative to polyunsaturated, fat on immune and inflammatory responses of adults with moderate hypercholesterolemia. J Lipid Res 2002; 43: 445-52

141. Esposito K, Marfella R, Ciotola M, Di Palo C, Giugliano F, Giugliano G, et al. Effect of a mediterranean-style diet on endothelial dysfunction and markers of vascular inflammation in the metabolic syndrome: a randomized trial. Jama 2004; 292: 1440-6 
142. Jenkins DJ, Kendall CW, Marchie A, Faulkner DA, Josse AR, Wong JM, et al. Direct comparison of dietary portfolio vs statin on C-reactive protein. Eur J Clin Nutr 2005; 59: 851-60

143. Lopez-Garcia E, Schulze MB, Fung TT, Meigs JB, Rifai N, Manson JE, et al. Major dietary patterns are related to plasma concentrations of markers of inflammation and endothelial dysfunction. Am J Clin Nutr 2004; 80: 1029-35

144. Ning B, Dial S, Sun Y, Wang J, Yang J, Guo L. Systematic and simultaneous gene profiling of 84 drug-metabolizing genes in primary human hepatocytes. J Biomol Screen 2008; 13: 194201

145. Hansel NN, Cheadle C, Diette GB, Wright J, Thompson KM, Barnes KC, et al. Analysis of CD4+ T-cell gene expression in allergic subjects using two different microarray platforms. Allergy 2008; 63: 366-9

146. van Leeuwen DM, Gottschalk RW, van Herwijnen MH, Moonen EJ, Kleinjans JC, van Delft JH. Differential gene expression in human peripheral blood mononuclear cells induced by cigarette smoke and its constituents. Toxicol Sci 2005; 86: 200-10

147. Stojakovic M, Krzesz R, Wagner AH, Hecker M. CD154-stimulated GM-CSF release by vascular smooth muscle cells elicits monocyte activation--role in atherogenesis. J Mol Med 2007; 85: 1229-38

148. Llorente-Cortes V, Royo T, Juan-Babot O, Badimon L. Adipocyte differentiation-related protein is induced by LRP1-mediated aggregated LDL internalization in human vascular smooth muscle cells and macrophages. J Lipid Res 2007; 48: 2133-40

149. Haymore BR, Parks JR, Oliver TG, Glister BC. Hypertriglyceridemia. Hospital Physician 2005; 17-24

150. Dutch Cholesterol Consensus. Syllabus behandeling en preventie van coronaire hartziekten door verlaging van de plasmaconcentratie. In. Book Syllabus behandeling en preventie van coronaire hartziekten door verlaging van de plasmaconcentratie, 1998; Utrecht: Centraal Begeleidingsorgaan voor de Intercollegiale Toetsing.

151. Matthews DR, Hosker JP, Rudenski AS, Naylor BA, Treacher DF, Turner RC. Homeostasis model assessment: insulin resistance and beta-cell function from fasting plasma glucose and insulin concentrations in man. Diabetologia 1985; 28: 412-9

152. Shine B, de Beer FC, Pepys MB. Solid phase radioimmunoassays for human C-reactive protein. Clin Chim Acta 1981; 117: 13-23

153. Packard CJ. Triacylglycerol-rich lipoproteins and the generation of small, dense low-density lipoprotein. Biochem Soc Trans 2003; 31: 1066-9

154. Cohn JS, Marcoux C, Davignon J. Detection, quantification, and characterization of potentially atherogenic triglyceride-rich remnant lipoproteins. Arterioscler Thromb Vasc Biol 1999; 19: 2474-86

155. Hallikainen M, Lyyra-Laitinen T, Laitinen T, Agren JJ, Pihlajamaki J, Rauramaa R, et al. Endothelial function in hypercholesterolemic subjects: Effects of plant stanol and sterol esters. Atherosclerosis 2006; 188: 425-32

156. Relas H, Gylling $\mathrm{H}$, Miettinen TA. Effect of stanol ester on postabsorptive squalene and retinyl palmitate. Metabolism 2000; 49: 473-8

157. Castro Cabezas M, de Vries JH, Van Oostrom AJ, lestra J, van Staveren WA. Effects of a stanol-enriched diet on plasma cholesterol and triglycerides in patients treated with statins. J Am Diet Assoc 2006; 106: 1564-9

158. Normen L, Johnsson M, Andersson H, van Gameren Y, Dutta P. Plant sterols in vegetables and fruits commonly consumed in Sweden. Eur J Nutr 1999; 38: 84-9

159. Grandgirard A, Martine L, Joffre C, Juaneda P, Berdeaux O. Gas chromatographic separation and mass spectrometric identification of mixtures of oxyphytosterol and oxycholesterol derivatives application to a phytosterol-enriched food. J Chromatogr A 2004; 1040: 239-50

160. Plat J, Beugels I, Gijbels MJ, de Winther MP, Mensink RP. Plant sterol or stanol esters retard lesion formation in LDL receptor-deficient mice independent of changes in serum plant sterols. J Lipid Res 2006; 47: 2762-71 
161. Ando M, Tomoyori H, Imaizumi K. Dietary cholesterol-oxidation products accumulate in serum and liver in apolipoprotein E-deficient mice, but do not accelerate atherosclerosis. $\mathrm{Br}$ J Nutr 2002; 88: 339-45

162. Sevanian A, Hodis HN, Hwang J, McLeod LL, Peterson H. Characterization of endothelial cell injury by cholesterol oxidation products found in oxidized LDL. J Lipid Res 1995; 36: 1971-86

163. Clare K, Hardwick SJ, Carpenter KL, Weeratunge N, Mitchinson MJ. Toxicity of oxysterols to human monocyte-macrophages. Atherosclerosis 1995; 118: 67-75

164. Peng SK, Tham P, Taylor CB, Mikkelson B. Cytotoxicity of oxidation derivatives of cholesterol on cultured aortic smooth muscle cells and their effect on cholesterol biosynthesis. Am J Clin Nutr 1979; 32: 1033-42

165. Christ M, Luu B, Mejia JE, Moosbrugger I, Bischoff P. Apoptosis induced by oxysterols in murine lymphoma cells and in normal thymocytes. Immunology 1993; 78: 455-60

166. Peng SK, Taylor CB, Hill JC, Morin RJ. Cholesterol oxidation derivatives and arterial endothelial damage. Atherosclerosis 1985; 54: 121-33

167. Matthias D, Becker CH, Godicke W, Schmidt R, Ponsold K. Action of cholestane-3 beta,5 alpha, 6 beta-triol on rats with particular reference to the aorta. Atherosclerosis 1987; 63: 115-24

168. Davis JB, Bowyer DE. Macrophages modify beta-VLDL by proteolysis and enhance subsequent lipid accumulation in arterial smooth muscle cells. Atherosclerosis 1989; 77 : 203-8

169. Cader AA, Steinberg FM, Mazzone T, Chait A. Mechanisms of enhanced macrophage apoE secretion by oxidized LDL. J Lipid Res 1997; 38: 981-91

170. Hulten LM, Lindmark H, Diczfalusy U, Bjorkhem I, Ottosson M, Liu Y, et al. Oxysterols present in atherosclerotic tissue decrease the expression of lipoprotein lipase messenger RNA in human monocyte-derived macrophages. J Clin Invest 1996; 97: 461-8

171. Jovinge S, Ares MP, Kallin B, Nilsson J. Human monocytes/macrophages release TNF-alpha in response to Ox-LDL. Arterioscler Thromb Vasc Biol 1996; 16: 1573-9

172. Liu Y, Hulten LM, Wiklund O. Macrophages isolated from human atherosclerotic plaques produce IL-8, and oxysterols may have a regulatory function for IL-8 production. Arterioscler Thromb Vasc Biol 1997; 17: 317-23

173. Turchetto E, Lercker G, Bortolomeazzi R. Oxisterol determination in selected coffees. Toxicol Ind Health 1993; 9: 519-27

174. Dutta PC. Studies on phytosterol oxides. II: Content in some vegetable oils and in french fries prepared in these oils. JAOCS 1997; 74: 659-666

175. Zunin P, Calcagno C, Evangelisti F. Sterol oxidation in infant milk formulas and milk cereals. J Dairy Res 1998; 65: 591-8

176. Dutta PC. Studies on phytosterol oxides. I: Effect of storage on the content in potato chips prepared in different vegetable oils. JAOCS 1997; 74: 647-657

177. Breuer O, Bjorkhem I. Use of an $18 \mathrm{O} 2$ inhalation technique and mass isotopomer distribution analysis to study oxygenation of cholesterol in rat. Evidence for in vivo formation of 7-oxo-, 7 beta-hydroxy-, 24-hydroxy-, and 25-hydroxycholesterol. J Biol Chem 1995; 270: 20278-84

178. Hansson GK. Inflammation, atherosclerosis, and coronary artery disease. N Engl J Med 2005; 352: 1685-95

179. Robinson JG, Stone NJ. Antiatherosclerotic and antithrombotic effects of omega-3 fatty acids. Am J Cardiol 2006; 98: 39i-49i

180. Davidson MH. Mechanisms for the hypotriglyceridemic effect of marine omega-3 fatty acids. Am J Cardiol 2006; 98: 27i-33i

181. Sadiq Butt M, Tahir-Nadeem M, Khan MK, Shabir R, Butt MS. Oat: unique among the cereals. Eur J Nutr 2008; 47: 68-79 
182. Davy BM, Davy KP, Ho RC, Beske SD, Davrath LR, Melby CL. High-fiber oat cereal compared with wheat cereal consumption favorably alters LDL-cholesterol subclass and particle numbers in middle-aged and older men. Am J Clin Nutr 2002; 76: 351-8

183. Torronen R, Kansanen L, Uusitupa M, Hanninen O, Myllymaki O, Harkonen H, et al. Effects of an oat bran concentrate on serum lipids in free-living men with mild to moderate hypercholesterolaemia. Eur J Clin Nutr 1992; 46: 621-7

184. Whyte JL, McArthur R, Topping D, Nestel P. Oat bran lowers plasma cholesterol levels in mildly hypercholesterolemic men. J Am Diet Assoc 1992; 92: 446-9

185. Kim SY, Song HJ, Lee YY, Cho KH, Roh YK. Biomedical issues of dietary fiber beta-glucan. J Korean Med Sci 2006; 21: 781-9

186. Dawson TC, Kuziel WA, Osahar TA, Maeda N. Absence of CC chemokine receptor-2 reduces atherosclerosis in apolipoprotein E-deficient mice. Atherosclerosis 1999; 143: 20511

187. Palazzo AJ, Jones SP, Girod WG, Anderson DC, Granger DN, Lefer DJ. Myocardial ischemia-reperfusion injury in CD18- and ICAM-1-deficient mice. Am J Physiol 1998; 275: H2300-7

188. Bisoendial RJ, Kastelein JJ, Peters SL, Levels JH, Birjmohun R, Rotmans Jl, et al. Effects of CRP infusion on endothelial function and coagulation in normocholesterolemic and hypercholesterolemic subjects. J Lipid Res 2007; 48: 952-60

189. Sugiyama M, Ohashi M, Takase H, Sato K, Ueda R, Dohi Y. Effects of atorvastatin on inflammation and oxidative stress. Heart Vessels 2005; 20: 133-6

190. King DE, Egan BM, Geesey ME. Relation of dietary fat and fiber to elevation of C-reactive protein. Am J Cardiol 2003; 92: 1335-9

191. King DE, Egan BM, Woolson RF, Mainous AG, 3rd, Al-Solaiman Y, Jesri A. Effect of a highfiber diet vs a fiber-supplemented diet on C-reactive protein level. Arch Intern Med 2007; 167: 502-6

192. King DE, Mainous AG, 3rd, Egan BM, Woolson RF, Geesey ME. Effect of psyllium fiber supplementation on C-reactive protein: the trial to reduce inflammatory markers (TRIM). Ann Fam Med 2008; 6: 100-6

193. Esposito K, Giugliano D. Diet and inflammation: a link to metabolic and cardiovascular diseases. Eur Heart J 2006; 27: 15-20

194. Expert panel on detection e, and treatment of high blood cholesterol in adults. Executive summary of the third report of the national cholesterol education program (NCEP) expert panel on detection, evaluation, and treatment of high blood cholesterol in adults (Adult treatment panel III). Jama 2001; 285: 2486-2497

195. Jenkins DJ, Kendall CW, Marchie A, Faulkner DA, Wong JM, de Souza R, et al. Direct comparison of a dietary portfolio of cholesterol-lowering foods with a statin in hypercholesterolemic participants. Am J Clin Nutr 2005; 81: 380-7

196. Jakulj L, Trip MD, Sudhop T, von Bergmann K, Kastelein JJ, Vissers MN. Inhibition of cholesterol absorption by the combination of dietary plant sterols and ezetimibe: effects on plasma lipid levels. J Lipid Res 2005; 46: 2692-8

197. Moghadasian MH, McManus BM, Pritchard PH, Frohlich JJ. "Tall oil"-derived phytosterols reduce atherosclerosis in ApoE-deficient mice. Arterioscler Thromb Vasc Biol 1997; 17: 119-26

198. Moghadasian MH, McManus BM, Godin DV, Rodrigues B, Frohlich JJ. Proatherogenic and antiatherogenic effects of probucol and phytosterols in apolipoprotein E-deficient mice: possible mechanisms of action. Circulation 1999; 99: 1733-9

199. Moghadasian MH, Godin DV, McManus BM, Frohlich JJ. Lack of regression of atherosclerotic lesions in phytosterol-treated apo E-deficient mice. Life Sci 1999; 64: 1029-36

200. Moghadasian MH. Dietary phytosterols reduce probucol-induced atherogenesis in apo E-KO mice. Atherosclerosis 2006; 188: 28-34

201. Weingartner O, Lutjohann D, Ji S, Weisshoff N, List F, Sudhop T, et al. Vascular effects of diet supplementation with plant sterols. J Am Coll Cardiol 2008; 51: 1553-61 
202. Glueck CJ, Speirs J, Tracy T, Streicher P, Illig E, Vandegrift J. Relationships of serum plant sterols (phytosterols) and cholesterol in 595 hypercholesterolemic subjects, and familial aggregation of phytosterols, cholesterol, and premature coronary heart disease in hyperphytosterolemic probands and their first-degree relatives. Metabolism 1991; 40: 842-8

203. Rajaratnam RA, Gylling H, Miettinen TA. Independent association of serum squalene and noncholesterol sterols with coronary artery disease in postmenopausal women. J Am Coll Cardiol 2000; 35: 1185-91

204. Sudhop T, Gottwald BM, von Bergmann K. Serum plant sterols as a potential risk factor for coronary heart disease. Metabolism 2002; 51: 1519-21

205. Wilund KR, Yu L, Xu F, Vega GL, Grundy SM, Cohen JC, et al. No association between plasma levels of plant sterols and atherosclerosis in mice and men. Arterioscler Thromb Vasc Biol 2004; 24: 2326-32

206. Miettinen TA, Railo M, Lepantalo M, Gylling H. Plant sterols in serum and in atherosclerotic plaques of patients undergoing carotid endarterectomy. J Am Coll Cardiol 2005; 45: 1794 801

207. Assmann G, Cullen P, Erbey J, Ramey DR, Kannenberg F, Schulte H. Plasma sitosterol elevations are associated with an increased incidence of coronary events in men: results of a nested case-control analysis of the Prospective Cardiovascular Munster (PROCAM) study. Nutr Metab Cardiovasc Dis 2006; 16: 13-21 
SUMMARY 
Cardiovascular disease (CVD) is the leading cause of death in many parts of the world. The conventional risk factors for CVD include smoking, unhealthy dietary habits, physical inactivity, being overweight, hypertension, and abnormal blood lipid levels. As these factors are strongly influenced by behavioral factors, there is now broad consensus that encouraging people to adopt a healthy lifestyle (i.e. not smoking, being physically active, and eating a healthy diet) is fundamental to reduce CVD risk. The inability of the established risk factors to account for all the variations in CVD between and within populations has further led to the emergence of a number of novel CVD risk markers. These factors include oxidative stress, infectious agents, and increased levels of small-dense low-density lipoproteins (sdLDL), C-reactive protein (CRP), and oxysterols.

Dietary therapy is effective to reduce CVD risk and mortality, associated with high serum LDL cholesterol levels. Functional foods made to lower the atherogenic LDL particles, and thereby the risk for CVD, are therefore gaining a prominent position in dietary guidelines. Such foods contain (or lack) one or more functional components and therefore provide positive health effects beyond their traditional nutritional value. Examples of such food components with FDA-approved health claims are the water-soluble fiber $\beta$-glucan from oats and barley, and plant sterols and stanols. These functional foods are in particular helpful for those individuals with elevated serum LDL cholesterol levels, if the product is substituted for a standard product and eaten as part of a cholesterol-lowering diet and in conjunction with a healthy lifestyle.

The aim of the studies described in this thesis was to investigate the role of (combining) different dietary components, such as oat $\beta$-glucan, plant stanols, and oxy(phyto)sterols in managing CVD risk. Within this objective, we have formulated several specific research questions, as described below.

The combined intake of oat $\beta$-glucan and plant stanols may be more effective than either component alone. In our first study (Chapter 3), we therefore investigated the effects of a simultaneous intake of oat $\beta$-glucan and plant stanols on lipid metabolism in mildly hypercholesterolemic volunteers. In a randomized, controlled, 3-period crossover study, 40 mildly hypercholesterolemic men and women received for 4 weeks either control muesli (5 $\mathrm{g}$ of wheat fiber), $\beta$-glucan enriched muesli (5 g of oat $\beta$-glucan), or combination muesli [5 $\mathrm{g}$ of oat $\beta$-glucan plus $1.5 \mathrm{~g}$ of plant stanols (as their fatty acids)]. The $\beta$-glucan enriched muesli effectively lowered serum LDL cholesterol concentrations by $5 \%$. Addition of plant stanols to this muesli further lowered serum LDL cholesterol levels by $4 \%$. The muesli rich in $\beta$-glucan increased bile acid synthesis and decreased cholesterol absorption, in line with the proposed cholesterol-lowering mechanism of viscous soluble fibers. The addition of plant stanols did not influence bile acid synthesis, but decreased cholesterol absorption and raised cholesterol synthesis, also in correspondence to their suggested working 
mechanism. We concluded that muesli rich in oat $\beta$-glucan effectively lowered serum LDL cholesterol concentrations. Addition of plant stanols to the $\beta$-glucan-enriched muesli further lowered LDL cholesterol levels, although less than predicted. We therefore speculated that the presence of the viscous soluble fiber oat $\beta$-glucan or the food matrix may modulate the cholesterol-lowering efficacy of the plant stanols.

Besides their hypocholesterolemic effects, in vitro and animal studies have suggested that oat $\beta$-glucan and phytosterols may also affect inflammatory processes. To explore whether the effects of oat $\beta$-glucan and plant stanols on CVD risk may be mediated in part by affecting inflammation, we examined in Chapter 4 the effects of these functional food ingredients on inflammatory markers. Pro-inflammatory cytokine (IL-6, IL-8, and TNF $\alpha$ ) production by PBMC and whole blood after LPS stimulation did not differ between the treatments. Also plasma levels of hs-CRP, a marker for low-grade systemic inflammation, were the same. No effects of oat $\beta$-glucan consumption on gene expression (human atherosclerosis PCR array) of PBMC were observed, while only 3 out of 84 genes from the atherosclerotic risk panel were differentially expressed after addition of the plant stanols. We concluded that consumption of oat $\beta$-glucan with or without plant stanols effectively lowered LDL cholesterol levels, but did not influence inflammatory parameters in slightly hypercholesterolemic subjects.

The link between high serum LDL cholesterol levels and CVD has been clearly established. However, evidence is accumulating that high levels of triacylglycerols (TAG), also known as hypertriglyceridemia, and low levels of HDL cholesterol are also causally related to CVD. In a second study (Chapter 5), we therefore investigated the effects of plant stanols on the serum lipoprotein profile in 26 men and women, especially selected for elevated fasting serum TAG concentrations. After a 1-week run-in period, during which control margarine (containing $60 \%$ absorbable fats) was used, subjects were randomized to receive daily either control margarine or plant stanol-enriched margarine $[2.5 \mathrm{~g} / \mathrm{d}$ of plant stanols (as esters)] for 3 weeks. Consumption of plant stanols significantly decreased serum total and LDL cholesterol levels. A significant interaction between baseline TAG values and response-to-treatment was found. Supplementation of plant stanols lowered serum TAG concentrations, particularly in subjects with high baseline TAG concentrations $(>2.3$ $\mathrm{mmol} / \mathrm{L}$ ). Additionally, a significant interaction between baseline number of total LDL particles and plant stanol intake was found. Consumption of plant stanols lowered the total number of LDL particles, primarily in subjects with elevated baseline values, and this was mainly due to a decrease in the sdLDL particles. We therefore concluded that consumption of plant stanols not only lowered serum LDL cholesterol, but also serum TAG concentrations, especially in subjects with elevated serum TAG concentrations. 
Oxysterols are suggested to be atherogenic and accordingly may also play an active role in the pathogenesis of CVD. Plant sterols structurally resemble cholesterol and the presence of one or more unsaturated bonds makes plant sterols susceptible to oxidation as well. However, it is unknown whether oxyphytosterols are atherogenic, as has been suggested for oxysterols. In a third study (Chapter 6), we therefore examined the effects of oxysterols and oxyphytosterols on serum lipoproteins and atherosclerotic lesion development in female transgenic mice. After a 2-week run-in period, 33 female heterozygous LDL receptor-deficient (LDLR+/-) mice were randomized to receive either control diet (atherogenic diet), oxysterol diet (control diet with $0.025 \%$ oxysterols), or oxyphytosterol diet (control diet with $0.025 \%$ oxyphytosterols) for 35 weeks. At the end of the experiment, serum cholesterol concentrations did not differ between the treatments. Also cholesterol exposure and lipoprotein profiles were similar. Nevertheless, a shift toward more severe lesions was found after the oxysterol and oxyphytosterols diets compared to the control diet (no difference between oxysterols and oxyphytosterols). Lesion size and collagen content did however not differ between the different treatments. We concluded that not only dietary oxysterols, but also dietary oxyphytosterols may promote the development of atherosclerosis in LDLR+/- mice, primarily by a shift toward more severe atherosclerotic lesions.

Taken together, functional foods enriched with the viscous soluble fiber oat $\beta$-glucan and plant stanols effectively lower the atherogenic LDL particles, and thereby the risk profile for CVD. However, the combined intake of oat $\beta$-glucan and plant stanols lowered LDL cholesterol less than expected, possibly because of interference of the viscous soluble fiber with the plant stanol efficacy. Modulation of inflammatory markers may also be a valuable step in managing CVD risk, but consumption of oat $\beta$-glucan and plant stanols did not influence markers related to inflammation within a period of 4 weeks. Possibly, a more rigorous or longer-lasting change in dietary patterns is needed to achieve significant effects on markers of inflammation. Recent findings have highlighted not only elevated LDL cholesterol, but also elevated TAG levels as an important risk factor for CVD. We now found that plant stanols not only lowered LDL cholesterol, but also serum TAG levels, particularly in subjects with high baseline TAG levels. Oxysterols have also emerged as a risk factor for CVD. Phytosterols may undergo similar oxidative processes, and therefore oxyphytosterols may have the same atherogenic potential. In our animal study, consumption of oxysterols and oxyphytosterols promoted atherosclerotic lesion development by a shift toward more severe lesions. How these results compare to the human situation, warrants further study. 


\section{SAMENVATTING}


Hart- en vaatziekten (HVZ) vormen de belangrijkste doodsoorzaak in de Westerse wereld. De traditionele risicofactoren voor het verkrijgen van HVZ zijn: roken, ongezonde voeding, lichamelijke inactiviteit, overgewicht, hypertensie en een ongunstig lipoproteïnenprofiel. Om het hart en de bloedvaten gezond te houden, is het dus noodzakelijk om het risico op HVZ te verlagen (niet roken, voldoende beweging, gezonde voeding). De afgelopen jaren zijn er echter een aantal nieuwe factoren gevonden die ook geassocieerd zijn met een verhoogd risico op HVZ, zoals oxidatieve stress, infecties, en verhoogde serum concentraties van de schadelijke kleine LDL-deeltjes (sdLDL), CRP, en oxysterolen.

De huidige voedingsrichtlijnen om het risico voor HVZ te verlagen zijn met name gericht op het verlagen van het LDL-cholesterolgehalte of van de verhouding totaal cholesterol/HDL-cholesterol. Echter, ondanks strikte dieettrouw, zal het lipoproteïnenprofiel voor een groot deel van de bevolking ongunstig blijven. Voor deze mensen bieden de zogenaamde functionele voedingsmiddelen nieuwe mogelijkheden. Functionele voedingsmiddelen zijn voedingsmiddelen waarin de hoeveelheid of de beschikbaarheid van een bepaald bestanddeel is veranderd, of waaraan een component is toegevoegd, dan wel waaruit een component is verwijderd. De consumptie van een dergelijk voedingsmiddel kan dan leiden tot een verbeterde gezondheid en/of een verminderd risico op ziekte. Voorbeelden van functionele voedingsmiddelen om het LDL-cholesterol te verlagen zijn producten die verrijkt zijn met plantenstanolen of met de visceuze voedingsvezel -glucan uit haver of gerst.

Het onderzoek dat in dit proefschrift wordt beschreven is gericht op de rol (van het combineren) van verschillende voedingscomponenten, zoals $\beta$-glucan uit haver, plantenstanolen en oxy(fyto)sterolen in het cardiovasculair risicomanagement. Om deze vraagstelling te beantwoorden werden onderstaande studies uitgevoerd.

Het tegelijktijdig consumeren van $\beta$-glucan uit haver en plantenstanolen kan effectiever zijn dan het consumeren van de afzonderlijke componenten. In een eerste studie (Hoofdstuk 3) onderzochten we daarom de effecten van een gelijktijdige consumptie van $\beta$-glucan uit haver en plantenstanolen op de vetstofwisseling van hypercholesterolemische vrijwilligers. In een gerandomiseerde, placebo-gecontroleerde studie consumeerden 40 hypercholesterolemische mannen en vrouwen gedurende 4 weken dagelijks ofwel controle-muesli (5 g tarwevezel), $\beta$-glucan-verrijkte muesli (5 g $\beta$-glucan uit haver) of een combinatiemuesli [5 g $\beta$-glucan uit haver plus $1.5 \mathrm{~g}$ plantenstanolen (in de vorm van esters)]. De $\beta$ glucan-verrijkte muesli verlaagde het serum LDL-cholesterolgehalte met $5 \%$. Het toevoegen van plantenstanolen aan deze muesli zorgde voor een extra daling in het serum LDLcholesterolgehalte van $4 \%$. Voor wat betreft het werkingsmechanisme werd gevonden dat $\beta$-glucan uit haver de galzoutsynthese verhoogde en de cholesterolabsorptie verlaagde. Het toevoegen van plantenstanolen had geen invloed op de galzoutsynthese, maar verlaagde 
wel de cholesterolabsorptie en verhoogde de cholesterolsynthese. We concludeerden dat muesli verrijkt met $\beta$-glucan uit haver het serum LDL-cholesterolgehalte verlaagde. Hoewel het toevoegen van plantenstanolen aan deze muesli een verdere daling bewerkstelligde, was de daling minder sterk dan verwacht. Het is mogelijk dat de visceuze voedingsvezel $\beta$ glucan of de voedingsmatrix een negatief effect had op het cholesterolverlagende effect van de plantenstanolen.

In vitro en dierstudies suggereren dat $\beta$-glucan uit haver en plantenstanolen naast het LDLcholesterolgehalte ook ontstekingsparameters kunnen beïnvloeden. Om te onderzoeken of de invloed van deze functionele voedingsmiddelen op het risico op HVZ (gedeeltelijk) verloopt via het ontstekingsproces, onderzochten we in Hoofdstuk 4 de effecten van $\beta$ glucan uit haver en plantenstanolen op ontstekingsparameters. De pro-inflammatoire cytokineproductie (IL-6, IL-8, TNF- $\alpha$ ) door perifere mononucleaire bloedcellen (PBMC) en volbloed na LPS-stimulatie verschilden niet tussen de interventiegroepen. Ook het serum hs-CRP-gehalte, een marker voor chronische ontsteking, was gelijk voor alle groepen. Het consumeren van de $\beta$-glucan-verrijkte muesli had tevens geen effecten op de genexpressie van PBMC (humane atherosclerose-PCR array), terwijl slechts 3 van de 84 genen verschillend tot expressie kwamen na het toevoegen van plantenstanolen aan deze muesli. We concludeerden dan ook dat consumptie van $\beta$-glucan uit haver met of zonder plantenstanolen effectief het LDL-cholesterolgehalte verlaagde, maar dat het geen invloed had op ontstekingsparameters in hypercholesterolemische vrijwilligers.

Het verband tussen een verhoogd LDL-cholesterolgehalte en een verhoogd risico op HVZ is algemeen aanvaard. De laatste jaren wordt echter ook in toenemende mate melding gemaakt van een verband tussen een verlaagd HDL-cholesterolgehalte en een verhoogd triglyceridengehalte, ook wel hypertriglyceridemie genoemd, en een verhoogd risico op HVZ. We onderzochten daarom in een tweede studie (Hoofdstuk 5) de effecten van plantenstanolen op het serum lipidenprofiel in 26 mannen en vrouwen, geselecteerd op basis van een verhoogd triglyceridengehalte. Na een 1 week run-in periode (gebruik van een controle-margarine), werden de deelnemers willekeurig verdeeld over 2 groepen: controlegroep (controle-margarine) of stanol-groep [margarine verrijkt met $2.5 \mathrm{~g} / \mathrm{d}$ plantenstanolen (in de vorm van esters)]. De dagelijkse consumptie van plantenstanolen zorgde voor een significante verlaging van het serum LDL-cholesterolgehalte na 3 weken. Ook werd er een significante interactie teruggevonden tussen de startwaarde van het triglyceridengehalte en de inname van plantenstanolen: het consumeren van plantenstanolen verlaagde met name het serum triglyceridengehalte in personen met verhoogde startwaarden $(>2.3 \mathrm{mmol} / \mathrm{L})$. Tevens werd een significante interactie gevonden tussen het totale aantal LDL-cholesterol-deeltjes en de inname van plantenstanolen. De consumptie van plantenstanolen verlaagde het totale aantal LDL-deeltjes, met name in personen met 
verhoogde startwaarden, en dit grotendeels via een verlaging in de sdLDL-deeltjes. We concludeerden dat de consumptie van plantenstanolen niet alleen het serum LDLcholesterolgehalte, maar ook het triglyceridengehalte verminderde, en dit voornamelijk in personen met verhoogde startwaarden.

Oxysterolen zijn mogelijk ook atherogeen en kunnen daarom een actieve rol spelen bij het ontstaan van HVZ. Plantensterolen hebben een vergelijkbare structuur als cholesterol en de aanwezigheid van een of meerdere onverzadigde bindingen makt deze stoffen ook vatbaar voor oxidatie. Echter, het is nog niet bekend of oxyfytosterolen ook atherogeen zijn, zoals gesuggereerd wordt voor oxysterolen. We onderzochten daarom in een derde studie (Hoofdstuk 6) de effecten van oxysterolen en oxyfytosterolen op het serum lipidenprofiel en de atherosclerotische plaque-vorming in transgene muizen. Na 2 weken run-in werden 33 vrouwelijke heterozygote LDL-receptor deficiënte (LDLR+/-) muizen willekeurig verdeeld over 3 groepen: controlegroep (atherogeen controledieet), oxysterol-groep (controledieet met $0.025 \%$ oxysterolen), of de oxyfytosterol-groep (controledieet met $0.025 \%$ oxyfytosterolen). Na 35 weken verschilde het serum cholesterolgehalte niet tussen de verschillende dieetgroepen. Ook het lipoproteïnenprofiel was gelijk voor alle groepen. Echter werd er wel een verschuiving gevonden richting meer gevorderde laesies na consumptie van oxysterolen en oxyfytosterolen in vergelijking tot het controledieet (geen verschil tussen de oxysterolen en oxyfytosterolen). Laesiegrootte en de hoeveelheid collageen in de laesies verschilden niet tussen de 3 dieetgroepen. We concludeerden dat niet alleen oxysterolen uit de voeding, maar ook oxyfytosterolen de ontwikkeling van atherosclerose kunnen beïnvloeden in vrouwelijke LDLR+/- muizen, en dit met name via een verschuiving naar meer ernstige atherosclerotische laesies.

Als onderdeel van een verantwoord voedingspatroon, kunnen voedingsmiddelen verrijkt met plantenstanolen of met wateroplosbare voedingsvezels een bijdrage leveren tot het verbeteren van een ongunstig lipoproteïnenprofiel. Echter, gelijktijdige consumptie van deze twee functionele voedingscomponenten verlaagde het LDL-cholesterolgehalte minder sterk dan verwacht, waarschijnlijk door de interferentie van visceuze voedingsvezel met het cholesterolverlagende effect van de plantenstanolen. Het gunstig beïnvloeden van ontsteking kan ook een belangrijke bijdrage leveren aan het cardiovasculair risicomanagement, maar de consumptie van $\beta$-glucan uit haver en plantenstanolen had hier geen effect op. Mogelijk is een verandering van een volledig voedingspatroon in vergelijking tot slechts 2 voedingscomponenten nodig om effecten te bekomen op ontsteking. Recent onderzoek toonde aan dat naast een verhoogd cholesterolgehalte ook een verhoogd triglyceridengehalte een belangrijke risicofactor is voor HVZ. Consumptie van plantenstanolen gaf naast een verlaging in het LDL-cholesterolgehalte ook een reductie in de serum triglyceridenconcentraties, en dit met name in personen met verhoogde startwaarden. Ook 
oxysterolen worden gezien als een mogelijke risicofactor voor HVZ. Evenals cholesterol, kunnen ook plantensterolen worden geoxideerd. De consumptie van oxysterolen en oxyfytosterolen stimuleerden de atherosclerotische plaque-ontwikkeling door een verschuiving naar meer ernstige laesies. Hoe deze resultaten zich vertalen naar de menselijke situatie vraagt echter meer onderzoek. 



\section{APPENDIX}

\section{Dankwoord}

\section{Curriculum Vitae}

Publications

Abbreviations 



\section{DANKWOORD}

... en mijn hart bevat vele mooie herinneringen aan mijn promotietraject en dus ook een grote dankbaarheid. Dat verdient een woordje van dank!

Beste Ronald (prof. dr. ir. R.P. Mensink) en Jogchum (dr. J. Plat), jullie zou ik graag als eerste willen bedanken voor de mooie tijd onder jullie bewind. Voor mij was het promotietraject niet enkel een periode van wetenschapplijke groei, maar ook een ontplooiing op persoonlijk vlak [mede dankzij de Nederlandse collega's ;-)]. Bedankt voor de gegeven kansen, jullie wetenschappelijk enthousiasme/kriticisme, jullie flexibiliteit en natuurlijk voor de dagdagelijkse begeleiding.

De leden van de beoordelingscommissie, prof. dr. ir. W.H.M. Saris, dr. F.J.P.H. Brouns, prof. dr. em. G. Hornstra, dr. D. Lütjohann en prof. dr. C.D.A. Stehouwer, wil ik graag bedanken voor het kritisch evalueren van mijn proefschrift. Dear dr. D. Lütjohann, thank you for the critical evaluation of my thesis.

Beste proefpersonen, jullie bijdrage aan mijn onderzoek was onmisbaar! Bedankt voor jullie tijd en interesse. Het was altijd een plezier om met jullie samen te kunnen werken.

I would like to thank Raisio for providing the experimental products and their scientific support.

Dank aan alle collega's! Werken in een omgeving met toffe collega's is voor mij zeer belangrijk. Mijn kamergenootjes, Frank, Julian, Léonie, Marjolijn, Maurice, Myriam en Stefan, wil ik graag bedanken voor de gezellige sfeer en gesprekken. Marjolijn, we hebben ongeveer 4 jaar samen doorgebracht in die kleine ruimte en nooit een minder moment meegemaakt. Bedankt voor de aangename tijd en je luisterend oor. (N)Elke, Kirsten, Miriam en Pia, bedankt voor het ondersteunen van de humane studies en jullie bijdrage aan de gezelligheid. Carla, Frank, Jacqueline, Loek, Martine, de 2 Maurice-sen, Paul en natuurlijk Jos, bedankt voor de technische ondersteuning en ook jullie bijdrage aan de goede werksfeer. Inge, Ilona en Marion, ik wil jullie graag bedanken voor de hulp bij de muizenstudie. Het was voor mij een zeer leerrijke ervaring om met deze beestjes om te gaan. Claudia, Désiree, Ilona, Kerstin en Larisa, jullie waren super secretaresses! Ruth, bedankt voor de fijne ritjes naar en weg van het werk. De ritten samen vlogen voorbij! 
Angelina, Arienne, Chantal, Florence, Gemma, Herman, Julia, Noud, Pascal, Petra, Stan en al de rest, BEDANKT!!

Mijn nieuwe collega's, en in het bijzonder mijn nieuwe kamergenootjes, bedankt voor de blijde ontvangst in de groep (op de kamer) en de mooie tijd die we al samen hebben gehad!

Zoals collega's hun steentje bijdragen aan een toffe werksfeer, zorgen familie (incl. schoonfamilie) en vrienden voor een aangename "levenssfeer". An, Lien en Silvy, alias jogvrollie, bedankt voor jullie trouw aan de joggroep. Op naar de volgende 3 jaren van sport en plezier! Fia, Katleen, Lien en Tamara, alias kakelbond, bedankt voor het maandelijkse gekakel. Vijf-jaren van gekakel schept een band! Sandra en Sophie, bedankt voor jullie jarenlange vriendschap! Kathy, Katrien, Kim, Karen, Ruth en Tessy, en al de rest van familie en vrienden, bedankt om er altijd te zijn! Door vriendschap wordt vreugde verdubbeld en verdriet gedeeld.

Mijn liefste paranimfjes, Fia en Julia, bedankt voor jullie steun en vriendschap. Jullie zijn aangename, bijzondere vrouwen! Ik ben trots dat jullie op deze dag aan mijn zijde staan!

Lieve Bart, onze wegen kruisten elkaar voor het eerst toen we 5 jaar oud waren en nadien nog vele malen. Uw eeuwige enthousiasme zal voor mij (en vele anderen) een inspiratiebron blijven.

Mama en papa, bedankt voor jullie onvoorwaardelijke steun! Ik denk dat kinderen hun ouders niet vaak genoeg kunnen bedanken. Lien en Kim, ook jullie bedankt voor de luisterende oortjes! Jullie zijn niet alleen zussen, maar ook vriendinnen voor het leven.

Allerliefste Joost, bedankt voor uw geloof in mij, uw steun, vriendschap, liefde, ... en uw geduld ;-). Samen bouwen we iets moois!

Elke (alias Belke voor de middenlob), 8 oktober 2008 


\section{CURRICULUM VITAE}

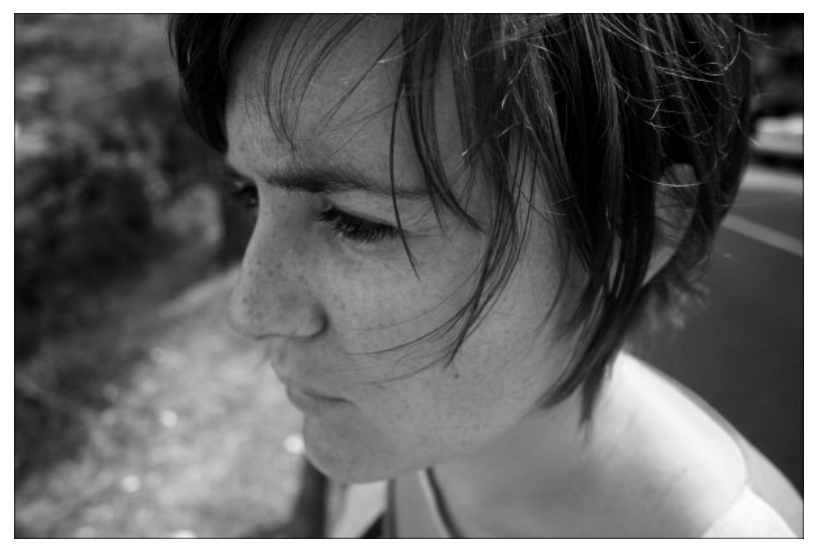

Elke Theuwissen was born on April the $14^{\text {th }}, 1979$ in Leuven. She completed high school at the Saint Augustine's Institute Bree in 1997 (major science-mathematics). In 2001, she graduated as Master of Biology (major molecular biology and physiology) at the Catholic University of Leuven (K.U.L.). At the same university, Elke also graduated as Master of Management after her studies of biology. Since 2003, she has been working on her PhD thesis at the Department of Human Biology, Maastricht University. Her thesis describes several studies in relation to "The role of functional food components in managing cardiovascular risk". Since February 2008, Elke has been employed as a postdoctoral fellow by VitaK, a research company closely associated with the CArdiovascular Research Institute Maastricht (CARIM). 



\section{PUBLICATIONS}

Theuwissen E, De Jong A, Plat J, Mensink RP. Plantensterolen en voedingsvezels: trendsetters voor 'functionele voedingsmiddelen'. Nederlands Tijdschrift voor Klinische Chemie en Laboratoriumgeneeskunde 2005; 30: 234-237.

Theuwissen E, Mensink RP. Water-soluble dietary fibers and cardiovascular disease. Physiol Behav. 2008; 94(2): 285-92.

Theuwissen E, Mensink RP. Simultaneous intake of $\beta$-glucan and plant stanol esters affects lipid metabolism in slightly hypercholesterolemic subjects. J Nutr. 2007; 137(3): 583-8.

Theuwissen E, Plat J, Mensink RP. Consumption of oat -glucan with or without plant stanol esters did not influence inflammatory markers in slightly hypercholesterolemic subjects. Mol Nutr Food Res. 2008; In press.

Theuwissen E, Plat J, van der Kallen CJH, van Greevenbroek MMJ, Mensink RP. Plant stanol consumption decreases serum triacylglycerols in subjects with overt hypertriglyceridemia. Submitted.

Theuwissen E, Plat J, Gijbels MJJ, van der Made I, Mensink RP. Oxyphytosterols increase the proportion of severe atherosclerotic lesions in LDLR+/- mice. Submitted.

\section{Abstracts}

Theuwissen E, Mensink RP. The effects of a simultaneous intake of dietary fiber plus plant stanol esters on concentrations of serum lipids in healthy subjects with mild to moderate hypercholesterolemia. Atherosclerosis. 2005; 6(1), Suppl: 123.

Theuwissen E, Plat J, Mensink RP. Effects of oat $\beta$-glucan with or without plant stanol esters on inflammatory parameters in slightly hypercholesterolemic subjects. Ann Nutr Metab. 2007; 51(1), Suppl 1: 56.

Plat J, Theuwissen E, Lütjohann D, Mensink RP. Do oxidized plant sterols underlie CHD risk in sitosterolemia patients? Gastroenterology. 2008; 134(4), Suppl 1: A406. 



\section{ABBREVIATIONS}

\begin{tabular}{|c|c|}
\hline $7 \alpha-\mathrm{OHC}$ & $7 \alpha$-hydroxy-4-cholesten-3-one \\
\hline GGT & $\gamma$-glutamyl transferase \\
\hline $\mathrm{ABC}$ & ATP-binding cassette transporter \\
\hline Acetyl-CoA & acetyl-coenzyme A \\
\hline ADFP & adipose differentiation-related protein \\
\hline ADRP & adipose differentiation-related protein \\
\hline APO & apolipoprotein \\
\hline AUC & area under the curve \\
\hline AZM & Academic Hospital Maastricht \\
\hline CETP & cholesteryl ester-transfer protein \\
\hline CHD5 & VE-cadherin \\
\hline COL3A1 & collagen type-3- $\alpha 1$ \\
\hline COPD & chronic obstructive pulmonary disease \\
\hline CSF2 & granulocyte-macrophage colony-stimulating factor \\
\hline CVD & cardiovascular disease \\
\hline DM2 & type 2 diabetes \\
\hline FABP3 & fatty acid-binding protein-3 \\
\hline $\mathrm{FCHL}$ & familial combined hyperlipidemia \\
\hline FDA & Food and Drug Administration \\
\hline FGA & fibrinogen \\
\hline FN1 & fibrinonectin-1 \\
\hline FPLC & fast protein liquid chromatography \\
\hline GMCSF & granulocyte-macrophage colony-stimulating factor \\
\hline GRAS & Generally Recognized as Safe \\
\hline HCAEC & human coronary artery endothelial cells \\
\hline $\mathrm{HDL}$ & high-density lipoprotein \\
\hline HMG-CoA & 3-hydroxy-3-methyl-glutaryl CoA reductase \\
\hline Hs-CRP & high-sensitivity C-reactive protein \\
\hline IDL & intermediate-density lipoprotein \\
\hline IHD & ischaemic heart disease \\
\hline IL & interleukin \\
\hline KDR & kinase insert domain receptor \\
\hline LAMA1 & laminin- $\alpha 1$ \\
\hline LCAT & lecithin-cholesterol acyltransferase \\
\hline LDL & low-density lipoprotein \\
\hline LDLR+/- & heterozygous LDL receptor-deficient \\
\hline LPA & $\operatorname{Lp}(\mathrm{a})$ \\
\hline LPL & lipoprotein lipase \\
\hline LPS & lipopolysaccharide \\
\hline MCP1 & monocyte chemotactic protein- 1 \\
\hline MMP3 & matrix metalloproteinase-3 \\
\hline
\end{tabular}


MS

NMR

NPC1L1

NPY

PBMC

PPARG

IN

SCFA

CHD

SMC

SPP1

TAG

TMB

VLDL metabolic syndrome

nuclear mass spectrometry

Niemann-Pick C-1 like-1 transporter

neuropeptide $Y$

peripheral blood mononuclear cells

peroxisome proliferative activated receptor- $\gamma$

RNA integrity number

short-chain fatty acids

coronary heart disease

smooth muscle cells

secreted phosphoprotein-1

triacylglycerols

tetramethylbenzidine

very low-density lipoprotein 